\title{
EXPLORING HALO SUBSTRUCTURE WITH GIANT STARS. X. EXTENDED DARK MATTER OR TIDAL DISRUPTION?: THE CASE FOR THE LEO I DWARF SPHEROIDAL GALAXY
}

\author{
Sangmo Tony Sohn, ${ }^{1,2,3,4}$ Steven R. Majewski, ${ }^{1}$ Ricardo R. Muñoz, ${ }^{1}$ William E. Kunkel, ${ }^{5}$ \\ Kathryn V. Johnston, ${ }^{6,7}$ James C. Ostheimer, ${ }^{1,3,8}$ Puragra Guhathakurta, ${ }^{9}$ \\ Richard J. Patterson, ${ }^{1,3}$ Michael H. Siegel, ${ }^{1,3,10}$ and Michael C. Cooper ${ }^{11}$ \\ Received 2005 December 5; accepted 2007 March 15
}

\begin{abstract}
We present a wide-field ( $\left.4.5 \mathrm{deg}^{2}\right)$ photometric and spectroscopic survey of the Leo I dwarf spheroidal (dSph) galaxy to explore its extended morphology and dynamics. To select Leo I red giant branch star candidates we exploit $M, T_{2}$, and DDO51 filter photometry; this yields $100 \%$ pure Leo I stars among more than $100 M<21.5$ Leo I giant candidates having previous or new Keck spectroscopy. The two-dimensional distribution of all photometric Leo I giant candidates is well fitted by a single-component King profile of limiting radius $13.4^{\prime}$ out to a major axis radial distance of $\sim 10^{\prime}$, but beyond this point the density profile shows an excess of stars along the major axis of the main body. This spatial configuration, together with a rather flat velocity dispersion profile and an asymmetric radial velocity distribution among Leo I members at large radii, supports a picture where Leo I has been tidally disrupted on one or two perigalactic passages about a massive Local Group member. We demonstrate this hypothesis using massfollows-light, $N$-body simulations of satellites in a Milky Way-like potential that reproduce the observed structural and dynamical properties of Leo I remarkably well. These models include $\sim 3 \times 10^{7}$ solar mass, tidally disrupting $\mathrm{dSphs}$ on bound orbits with rather high eccentricity (0.93-0.96) and small perigalactica (10-15 kpc). The simulations yield an observationally constrained orbit for Leo I without the measurement of its proper motion. Given the overall success of our satellite models to account for the observed properties of Leo I, we conclude that there is no need to invoke an extended dark matter halo around the satellite and that an overall modest $M / L$ for the satellite is consistent with the available data.
\end{abstract}

Subject headings: galaxies: evolution — galaxies: halos — galaxies: individual (Leo I) — galaxies: interactions — galaxies: photometry — galaxies: structure

Online material: color figures, machine-readable table

\section{INTRODUCTION}

\subsection{Motivations for a New Study of the Leo I System}

Modern theories of the evolution of structure in the universe that include cold dark matter (CDM) grow galaxies and clusters of galaxies and their dark halos through the accumulation of smaller subunits (e.g., White \& Rees 1978; Davis et al. 1985; Navarro et al. 1996, 1997; Moore et al. 1999). But the notion that

\footnotetext{
1 Department of Astronomy, University of Virginia, Charlottesville, VA 22903; srm4n@virginia.edu,rrm8f@virginia.edu,jco9w@virginia.edu, rjp0i@virginia.edu, mhs4p@virginia.edu.

2 Korea Astronomy and Space Science Institute, Daejeon 305-348, Korea; tonysohn@srl.caltech.edu.

3 Visiting Astronomer, Kitt Peak National Observatory, National Optical Astronomy Observatory ( NOAO). NOAO is operated by the Association of Universities for Research in Astronomy, Inc., under cooperative agreement with the National Science Foundation.

${ }^{4}$ Current address: California Institute of Technology, Pasadena, CA 91125; tonysohn@srl.caltech.edu.

5 Las Campanas Observatory, La Serena, Chile; kunkel@lcoeps1 .lco.cl.

6 Van Vleck Observatory, Wesleyan University, Middletown, CT 06459.

7 Current address: Department of Astronomy, Columbia University, New York, NY10027; kvj@astro.columbia.edu.

8 Current address: 1810 Kalorama Road NW, A3, Washington, DC 20009; jostheim@alumni.virginia.edu.

9 University of California Observatory/Lick Observatory, University of California, Santa Cruz, CA 95064; raja@ucolick.org.

${ }^{10}$ Current address: Department of Astronomy, University of Texas, Austin, TX 78712; siegel@astro.as.utexas.edu.

11 Department of Astronomy, University of California, Berkeley, CA 947203411; cooper@astron.berkeley.edu.
}

the Milky Way (MW) halo was built up by protracted infall of "protogalactic fragments" after an initial central collapse had already been established by Searle \& Zinn (1978) based purely on stellar population arguments. In CDM scenarios dwarf satellite galaxies represent the visible parts of (predominantly dark) subhalos; however, the number of dwarf galaxies discovered so far is several orders of magnitude less than the predicted number of subhalos made by CDM simulations (Kauffman et al. 1993; Klypin et al. 1999; Moore et al. 1999). This may be an indication that the majority of (especially smaller) subhalos either have not formed stars (Bullock et al. 2001) or have been destroyed (Hayashi et al. 2003), and that the visible satellites of today represent the high-mass end of the mass spectrum of DM subhalos (Stoehr et al. 2002; Hayashi et al. 2003) or those that were able to accrete substantial amounts of gas before reionization (Bullock et al. 2000; Benson et al. 2002; Taylor et al. 2004a).

On the other hand, dwarf galaxies exhibit some properties that may be inconsistent with the expected properties of CDM subhalos. For example, the flat central density profiles of dwarf spheroidal (dSph) galaxies are at odds with the cuspy interiors predicted by CDM (e.g., Navarro et al. 1996, 1997; Moore et al. 1998), although this might be reconciled by appealing to triaxial halos (Lokas 2002; Navarro 2004). Alternatively, warm dark matter (WDM) allows smaller central phase-space densities, and studies of dSph systems have provided important constraints on the properties of WDM species (Lin \& Faber 1983; Gerhard \& Spergel 1992; Goerdt et al. 2006; Strigari et al. 2006a). In addition to the problem of the central concentrations of satellites, their apparent 
alignments around parent halos, as has been observed in the MW and argued to be related to "dynamical families" of satellites (Kunkel 1979; Lynden-Bell 1982; Majewski 1994; Fusi Pecci et al. 1995; Lynden-Bell \& Lynden-Bell 1995; Palma et al. 2002), have also been used to question the viability of dwarf galaxies as putative subhalos (Kroupa et al. 2005; Kang et al. 2005). On the other hand, such alignments have also been reported in highresolution $N$-body, hierarchical structure simulations, where subhalos accrete along filaments (Libeskind et al. 2005; Zentner et al. 2005; Wang et al. 2005; Libeskind et al. 2007).

Despite uncertainties over the precise connection of observed satellite galaxies to the prevailing theoretical CDM models, it has become observationally clear that satellite galaxies (e.g., Ibata et al. 1995; Newberg et al. 2002; Majewski et al. 2003; Yanny et al. 2003; Crane et al. 2003; Rocha-Pinto et al. 2003) and even star clusters (Odenkirchen et al. 2001, 2003; Rockosi et al. 2002; Lee et al. 2004; Grillmair \& Johnson 2006; Belokurov et al. 2006a; Grillmair \& Dionatos 2006) could be significant contributors to the luminous halo of the MW and other galaxies. $N$-body simulations (Oh et al. 1995; Piatek \& Pryor 1995; Johnston et al. 1995, 1999, 2002; R. R. Muñoz et al. 2007, in preparation) show how a dwarf galaxy can experience tidal disruption in its outer parts during close encounters with the central potential well of MW-like, parent systems. Stars that escape the system will form tidal tails, like those observed in the Sagittarius (Sgr) system (e.g., Ibata et al. 1995; Newberg et al. 2002; Majewski et al. 2003; Belokurov et al. 2006b). Such extratidal stars can create "breaks" in the projected radial star count profiles of the satellites as the density law transitions from a steeply declining central density law for bound stars to a much more gradual decline at radii where unbound stars start to contribute significantly. Such breaks have been observed in several MW dSph satellites (Eskridge 1988a, 1988b; Irwin \& Hatzidimitriou 1995, hereafter IH95; Majewski et al. 2000a, 2003; Palma et al. 2003; Wilkinson et al. 2004; Westfall et al. 2006; Siegel \& Majewski 2006; Muñoz et al. 2006). For example, the radial density profiles of nearly every one of the then-known dSphs of the MW in the thorough IH95 study (see their Fig. 2) show an excess of stars with respect to the outer parts of their best-fitting model King profiles. In the particular case of Leo I, because of its extreme distance, the number of dSph stars relative to the background level in the outermost regions was too low to judge conclusively the significance of its apparent break and limited the ability of IH95 to explore the Leo I profile significantly past the King limiting radius with their photographic star counts. Undoubtedly, a radial surface density profile with better signal-to-noise ratio $(\mathrm{S} / \mathrm{N})$ and spatial coverage will help verify whether this extremely distant Galactic companion shows the break profile trait seen in other MW dSphs. Determining the structure of Leo I to large radii is a primary goal of the present study.

A second goal is to increase our understanding of the internal dynamics of Leo I. If dSph galaxies are the visible counterparts of the largest DM lumps (e.g., Stoehr et al. 2002), one might expect the process of tidal disruption to be inhibited or lessened in these massive subhalos. On the other hand, if dSphs can be proven to have tidal tails, the latter would place constraints on the dark matter components within these objects (Moore 1996). The Sgr $\mathrm{dSph}$ provides a vivid example of a tidally disrupting system for which the degree of disruption, as measured by the nature of the tails, has been used to constrain its dark matter content (Law et al. 2005); on the other hand, the Sgr system is sometimes considered to be an exception to the norm for dSph galaxies (Mateo et al. 1998, hereafter M98; Mateo 1998). That Sgr may not be an exceptional case is suggested by studies of possible tidal disrup- tion in other MW dSphs (Gould et al. 1992; Kuhn et al. 1996; Majewski et al. 2000a, 2006; Gómez-Flechoso \& MartínezDelgado 2003; Palma et al. 2003; Walcher et al. 2003; Westfall et al. 2006; Muñoz et al. 2005, 2006, 2007).

The effects of tidal disruption can be inferred not only by the existence of break in the density profiles of these other MW dSphs but also, more recently, by the velocity characteristics of the dSphs at large radii. The past decade has seen substantial progress in measuring radial velocities ( $\mathrm{RVs}$ ) for large numbers of stars in at least the more nearby MW dSph galaxies (Armandroff et al. 1995; Tolstoy et al. 2004; Wilkinson et al. 2004; Muñoz et al. 2005, 2006; Walker et al. 2006). These studies not only imply large central mass-to-light ratios in many of the satellites but also generally reveal relatively flat velocity dispersion profiles reaching into the break profile regions (Muñoz et al. 2005, 2006), a phenomenon reproducible in $N$-body simulations of disrupting, mass-followslight satellites (R. R. Muñoz et al. 2007, in preparation).

However, a tidal disruption interpretation of these velocity dispersion profiles to large radii is not unique based on the extant data because flat velocity dispersion profiles may also be accommodated to arbitrary radii by extending the DM halos in which the luminous dSphs are embedded. These "equilibrium models" imply mass-to-light ratios that increase with radius and boost the global ratio, in some cases quite substantially (e.g., Kleyna et al. 2002). Knowing whether mass follows light in dSphs or if their luminous components lie within extended dark halos is critical to establishing the regulatory mechanisms that have inhibited the formation of galaxies in all subhalos and the extent to which luminous satellites are vulnerable to disruption and populate the stellar halos of $L^{*}$ galaxies.

Leo I provides an interesting contrasting case to other dSphs in that (1) this particular satellite clearly has an unusual orbit that has protected it from but a few potential tidal encounters, and (2) unlike the apparently high $M / L$ systems (like Ursa Minor, Draco, and Carina), the previously measured mass-to-light ratio of Leo I is only $\sim 6 M_{\odot} L_{\odot, V}^{-1}$, i.e., at the low end of the $\mathrm{dSph} M / L$ scale. Of course, it is of interest to know whether and how these two traits of this satellite may relate, and whether the properties of Leo I, if explored more extensively, may lend new insights into the issues raised above. For example, as we show in this paper, new signatures found in the velocity distribution of Leo I stars at large radii (namely, an asymmetry) may provide a way to break the degeneracy in interpretations of the velocity dispersion profile for at least some dSphs.

The heart of our study is a new photometric survey covering $4.5 \mathrm{deg}^{2}$ of the sky centered on Leo I and new Keck spectroscopy of 105 more Leo I giant stars than available in the literature. As with other contributions in this series, we adopt a technique based on multicolor Washington filter imaging, including the use of the DDO51 filter centered on the gravity-sensitive $\mathrm{MgH}+\mathrm{Mg} b$ triplet spectral feature near $5150 \AA$ to identify giant stars associated with the Leo I dSph. We use bright K giants of Leo I as a tracer population of the structure of the dSph because (1) they are the most easily detected type of stars over large areas with the use of a midsize telescope; (2) the Washington+DDO51 separation technique has already been proven successful in the discrimination of metalpoor dSph giant stars from foreground metal-rich field dwarf stars and thereby to significantly increase the $\mathrm{S} / \mathrm{N}$ of the faint, diffuse outer parts of Local Group dSphs (e.g., Majewski et al. 2000a; Palma et al. 2003; Muñoz et al. 2005, 2006); and (3) these stars are accessible to spectroscopic membership and dynamical follow-up with the currently largest telescopes.

Because we are able to explore the Leo I dSph to large radii with much better $\mathrm{S} / \mathrm{N}$ than previous studies, we can rederive the 
structural parameters for Leo I ( $§ 4.1)$, which are important to assessing the $M / L$ using the traditional, King (1962) methodology based on the internal dynamics of the system. A newly derived $M / L$ for Leo I in this way is discussed in $\S 4.2$. We also explore the possible mass distribution for Leo I within the context of a tidal disruption scenario using $N$-body simulations of Leo I-like satellites orbiting an MW-like galaxy in $\S 6.2$.

The unusually high RV of Leo I at its extreme distance naturally leads to interesting questions about its specific orbit. There have been two studies that are in disagreement about potential orbits for Leo I. On one hand, Byrd et al. (1994) conclude that Leo I was once loosely bound to M31 and now is in an unbound, hyperbolic orbit about the MW. They estimate Leo I's MW perigalactic distance to be $\sim 70 \mathrm{kpc}$ and for this nearest approach to have occurred 2-4 Gyr ago. On the other hand, in an earlier study, Zaritsky et al. (1989, hereafter Z89) concluded that Leo I probably did not originate in the M31 system and that the most reasonable assumption is that Leo I is bound to the MW. An unexpected benefit of our study is that we have been able to derive new constraints on the Leo I orbit from detailed study of its structure and dynamics ( $(6.2)$, and we find reasonable agreement of these results with inferences about Leo I's orbit based on its star formation history.

Dwarf spheroidal galaxies, with other halo objects such as globular clusters and field stars, are also useful test particles for probing the large-scale mass distribution and total mass of our Galaxy. Despite much work, the spatial extent and total mass of the MW remain among the more poorly established Galactic parameters. While traditionally thought to be a "lesser sibling" to the Andromeda galaxy (M31) in terms of mass, some recent work (Côté et al. 2000; Evans \& Wilkinson 2000; Evans et al. 2000; Geehan et al. 2006; Seigar et al. 2006) suggests that, in fact, the MW edges out M31 as the most massive galaxy in the Local Group. With the traditional analyses, the mass estimate is sensitive to the inclusion or exclusion of one particular object, Leo I, because of its combined unusually large galactocentric $\mathrm{RV}\left(+177 \pm 3 \mathrm{~km} \mathrm{~s}^{-1} ; \mathrm{Z} 89\right)$ and great distance $(257 \pm 8 \mathrm{kpc}$; see $\S 1.2$ ). Estimates of the Galactic mass by Z89 varied by a factor of 3-4 depending on whether Leo I is considered as bound to the MW or not. While recent studies by Wilkinson \& Evans (1999) and Sakamoto et al. (2003) have decreased the dependency of MW mass estimates on including Leo I by using Bayesian likelihood methods and larger samples of halo objects, Leo I is still considered to be a determining factor for fine-tuning the results.

\subsection{Previous Photometric Studies of Leo I}

The Leo I dSph was discovered more than half a century ago by Harrington \& Wilson (1950) during the first Palomar sky survey. Due to its extreme distance and its angular proximity to the first-magnitude foreground star Regulus, photometric studies of Leo I have been difficult. Not until CCD arrays were developed were the first color-magnitude diagrams (CMD) of Leo I constructed. Fox \& Pritchet (1987), Reid \& Mould (1991), Lee et al. (1993), and Demers et al. (1994) presented early ground-based CMDs. Later, Caputo et al. (1999) and Gallart et al. (1999b) presented Leo I CMDs based on data taken with the Hubble Space Telescope (HST), and they reach the main-sequence turnoff (MSTO) of the oldest ( $>10$ Gyr) Leo I populations. These deep CMDs allowed detailed studies of the multiple stellar populations and the complex star formation history of Leo I (Gallart et al. 1999a). Leo I was thought to be unique among the MW satellite dSphs for not having a conspicuous horizontal branch (HB) population until a $12^{\prime} \times 12^{\prime}$ ground-based survey on Leo I by Held et al. (2000) revealed an extended HB structure in its CMD. More recently, Held et al. (2001) discovered more than 70 RR Lyrae variables with pulsational properties suggesting an intermediate Oosterhoff type similar to other dwarf galaxies in the Local Group (Siegel \& Majewski 2000 ; Cseresnjes 2001; Pritzl et al. 2002). The existence of both extended blue HB population and RR Lyrae stars suggests that Leo I is in fact similar to other local dSph galaxies in having a $>10$ Gyr population, likely formed in the initial collapse of the system. The extended star formation history of Leo I, which includes this initial starburst, followed by a quiescent phase and a new burst of star formation activity starting $\sim 7 \mathrm{Gyr}$ ago (Gallart et al. 1999a), may be intimately tied to its orbital dynamics, since close interactions between galaxies are known to be triggers of star formation.

The distance to Leo I has been derived using various photometric methods. Lee et al. (1993) used the tip of the red giant branch (TRGB) method to derive the Leo I distance modulus $(m-M)_{0}=22.18 \pm 0.11$, while Demers et al. (1994) used the apparent magnitudes of both red clump and carbon stars to estimate $(m-M)_{0}=21.56 \pm 0.25$. Held et al. (2001) used the mean magnitude of RR Lyrae variables in Leo I and derived $(m-M)_{0}=22.04 \pm 0.14$, and more recently Bellazzini et al. (2004) provided a new estimate of $(m-M)_{0}=22.02 \pm 0.13$ using the TRGB method. A weighted average of the distance modulus derived in the four studies gives $(m-M)_{0}=22.05 \pm 0.07$, which converts to a distance of $257 \pm 8 \mathrm{kpc}$. Throughout this study, we adopt these values.

The following sections include a presentation of the data from our photometric survey of Leo I ( $(2)$ and a description of the photometric identification of the Leo I giant star candidates $(\S 3)$. The two-dimensional distribution of Leo I giant star candidates is discussed in $\S 4$, and new morphological parameters for the $\mathrm{dSph}$ are derived. In $\S 5$ we present new Keck Observatory spectroscopy of a subsample of our Leo I giant candidates, and in $\S 6$ we discuss the implications of our results, making use of new $\mathrm{N}$-body simulations of a tidally disrupting $\mathrm{dSph}$ satellite galaxy that appear to generate similar Leo I properties to those we have observed. Finally, a summary of our work and conclusions are in $\S 7$.

\section{PHOTOMETRIC OBSERVATIONS AND DATA REDUCTION}

The images used in this study were obtained with the Mayall $4 \mathrm{~m}$ Telescope at Kitt Peak National Observatory (KPNO) during the nights of UT 1998 November 17 and 2002 May 2-5. We used the Mosaic I $8 \mathrm{~K} \times 8 \mathrm{~K}$ CCD, which has a pixel scale of $0.26^{\prime \prime}$ pixel $^{-1}$ resulting in a $36 \times 36 \mathrm{arcmin}^{2}$ field of view. The camera is an array of eight $2048 \times 4096 \mathrm{CCD}$ chips. We used the broadband Washington $M$, Harris $I$, and intermediate-band DDO5 1 filters. ${ }^{12}$ Our survey fields were selected to lie predominantly along the major axis of Leo I. The area to the north of Leo I was sampled, but a large part to the south of Leo I was avoided due to Regulus. All program fields were overlapped by $6^{\prime}$ with adjacent fields as a check on consistency of the photometry. Table 1 summarizes the basic information of the CCD fields used in this study.

The raw images were preprocessed using the ccdproc task in the IRAF MSCRED package. ${ }^{13}$ The flat-fielding was done with

\footnotetext{
12 Hereafter we denote the $I$ filter as $T_{2}$ filter since their response curves are nearly identical (see, e.g., Fig. 1 of Lejeune \& Buser 1996; discussion by Majewski et al. 2000a)

${ }^{13}$ IRAF is distributed by the National Optical Astronomy Observatory, which is operated by the Association of Universities for Research in Astronomy, Inc., under cooperative agreement with the National Science Foundation.
} 
TABLE 1

Leo I Observation Log

\begin{tabular}{|c|c|c|c|c|c|}
\hline \multirow[b]{2}{*}{ FIELD } & \multirow{2}{*}{$\begin{array}{c}\text { R.A. } \\
(\mathrm{J} 2000.0)\end{array}$} & \multirow{2}{*}{$\begin{array}{c}\text { DeCL. } \\
(\mathrm{J} 2000.0)\end{array}$} & \multicolumn{3}{|c|}{ Exposure Time (s)/Air Mass/FWHM $(\operatorname{arcsec})$} \\
\hline & & & $M$ & $T_{2}$ & DDO51 \\
\hline $\mathrm{C} \ldots$ & 100828.69 & +121817.2 & $70 / 1.16 / 1.2$ & $70 / 1.15 / 0.8$ & $700 / 1.13 / 0.9$ \\
\hline 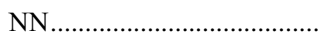 & 100829.27 & +131816.8 & $70 / 1.20 / 1.2$ & $70 / 1.19 / 0.9$ & $700 / 1.17 / 1.0$ \\
\hline EE & 101228.46 & +121808.7 & $70 / 1.29 / 1.1$ & $70 / 1.28 / 0.9$ & $700 / 1.25 / 1.0$ \\
\hline WW & 100428.91 & +121825.2 & $70 / 1.46 / 1.2$ & $70 / 1.44 / 1.0$ & $700 / 1.39 / 1.1$ \\
\hline 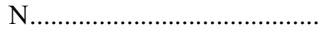 & 100828.12 & +124823.4 & $100 / 1.08 / 2.2$ & 200/1.09/1.8 & $1000 / 1.10 / 2.3$ \\
\hline ......... & 101028.12 & +12 1823.4 & $100 / 1.21 / 2.0$ & $200 / 1.23 / 1.6$ & $1000 / 1.27 / 1.8$ \\
\hline …………...... & 100628.11 & +12 1823.4 & $100 / 1.15 / 1.8$ & $200 / 1.16 / 2.0$ & $1000 / 1.19 / 1.7$ \\
\hline $\mathrm{NE}$ & 101028.12 & +124823.4 & $100 / 1.35 / 1.6$ & $200 / 1.38 / 1.6$ & $1000 / 1.44 / 1.7$ \\
\hline NW & 100628.55 & +124757.1 & $100 / 1.13 / 1.9$ & $200 / 1.14 / 2.1$ & $1000 / 1.20 / 1.7$ \\
\hline EEE & 101428.12 & +121823.4 & $100 / 1.09 / 1.1$ & $200 / 1.09 / 1.2$ & $1000 / 1.11 / 1.3$ \\
\hline 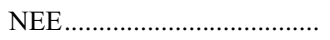 & 101228.11 & +124823.4 & $100 / 1.13 / 1.4$ & $200 / 1.14 / 1.4$ & $1000 / 1.16 / 1.3$ \\
\hline 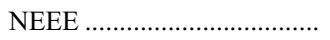 & 101428.12 & +124823.4 & $100 / 1.19 / 1.3$ & $200 / 1.20 / 1.5$ & $1000 / 1.24 / 1.8$ \\
\hline 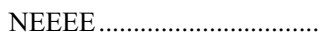 & 101628.12 & +124823.4 & $100 / 1.27 / 2.5$ & $200 / 1.29 / 2.2$ & $1000 / 1.34 / 1.8$ \\
\hline 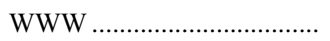 & 100230.17 & +12 1823.4 & $100 / 1.76 / 1.5$ & $200 / 1.81 / 1.5$ & $1000 / 1.95 / 1.4$ \\
\hline SWW …… & 100428.12 & +114823.4 & $100 / 1.26 / 1.6$ & $200 / 1.27 / 1.9$ & $1000 / 1.32 / 1.6$ \\
\hline SWWW & 100228.12 & +114823.4 & $100 / 1.48 / 1.8$ & $200 / 1.51 / 1.8$ & $1000 / 1.59 / 1.4$ \\
\hline
\end{tabular}

Note.-Units of right ascension are hours, minutes, and seconds, and units of declination are degrees, arcminutes, and arcseconds.

special care since the KPNO $T_{2}$ and DDO51 passband images are affected by a pupil image that produces an artifact that increases the amount of background light near the center. The $T_{2}$ and DDO51 images also suffer from fringing. We carefully followed the procedures described in Valdes (1998) to correct for these effects. Once the preprocessing was done, we split each Mosaic image into its eight subimages and performed stellar photometry via the DAOPHOT II/ALLSTAR (Stetson 1987) package. A point-spread function (PSF) was constructed using 25-50 bright and isolated stars for each subimage. The quality of each PSF was improved by removing neighboring stars and reconstructing the PSF iteratively. PSF magnitudes were derived using UNIX shell scripts based on ALLSTAR. The growth curve analysis package DAOGROW (Stetson 1990) was then used to correct for the missing light lying outside of the PSF tail (the aperture correction).

Measured instrumental magnitudes were calibrated against Geisler $(1990,1996)$ standards that were observed many times in different air-mass ranges over each observing run. We fit a transformation equation of the form

$$
\mathrm{MAG}-\operatorname{mag}=\alpha_{1}+\alpha_{2} X+\alpha_{3} C,
$$

where MAG is the Geisler standard magnitude, mag is the instrumental magnitude, $X$ is the observed air mass, and $C$ is the color index. We tested for terms in $X C$, and these were found to be negligible. The colors $M-T_{2}$ and $M-$ DDO51 were used in the equations derived for the $M$ and DDO51 filters, respectively. A color term for the $T_{2}$ filter was also found to be unnecessary. The rms of the solutions for the transformation equations was less than 0.01 mag in all three filters. For the 1998 observations, we used the existing transformations derived for other observations on these same nights by Ostheimer (2002). He notes that on the night of UT 1998 November 17 there were minor transparency variations, so this night's data are considered nonphotometric. We subsequently tied the 1998 photometry to that of 2002 May in the following manner: First, multiply measured stars in the overlapping regions among the 2002 observations were used to derive and apply frame-to-frame offsets, which in most cases were less than 0.01 mag for all three filters. This en- sures that all of the 2002 observations share the same photometric zero point. We then used stars in overlapping regions of the 1998 and 2002 observations to calculate the average magnitude offsets of each nonphotometric frame relative to the photometric ones, and the corresponding offsets were applied to all objects in each nonphotometric frame. We iterated these steps until all of the average offsets among all frames were less than $0.001 \mathrm{mag}$.

We note that images taken with mosaic CCDs such as those used in this study may suffer from chip-to-chip sensitivity differences that could make the color terms be chip dependent. Ostheimer (2002) found the chip-to-chip color terms of the KPNO Mosaic to be negligible by observing the standard fields in Washington and DDO51 filters on every CCD chip in the Mosaic array and by cross comparing the standard coefficients for each chip.

Astrometry of the detected objects was obtained by running the TFINDER task in the IRAF FINDER package and using USNOA2.0 catalog stars (Monet et al. 1998) as reference. Since Leo I is located at a high Galactic latitude $\left(b=49^{\circ}\right)$, the foreground reddening is not significant $[E(B-V)=0.037$, according to Schlegel et al. 1998]. It is, however, important to consider the variation of reddening within and across our program fields that may result in systematic differences in stellar magnitudes and colors. Each object in our data set has been corrected for reddening based on its Galactic coordinates (converted from the equatorial coordinates) and direct reference to the reddening map constructed by Schlegel et al. (1998). The $E(B-V)$ of all objects in our fields range from 0.031 to 0.050 .

Our survey covers a large spatial area, and our photometry is likely to contain a large number of galaxies. Bad columns, random cosmic rays, and photoelectron bleeding from saturated stars are other possible nonstellar contaminants. Nonstellar objects were eliminated from our photometry using the two DAOPHOT image quality diagnostic parameters SHARP and $\chi$. All sources beyond the range $-0.3<$ SHARP $<0.3$ were considered as objects with extreme nonstellar morphology and were rejected. In case of $\chi,{ }^{14}$

\footnotetext{
14 This is the ratio of the observed pixel-to-pixel scatter from the image profile compared to the expected pixel-to-pixel scatter from a model stellar image profile.
} 


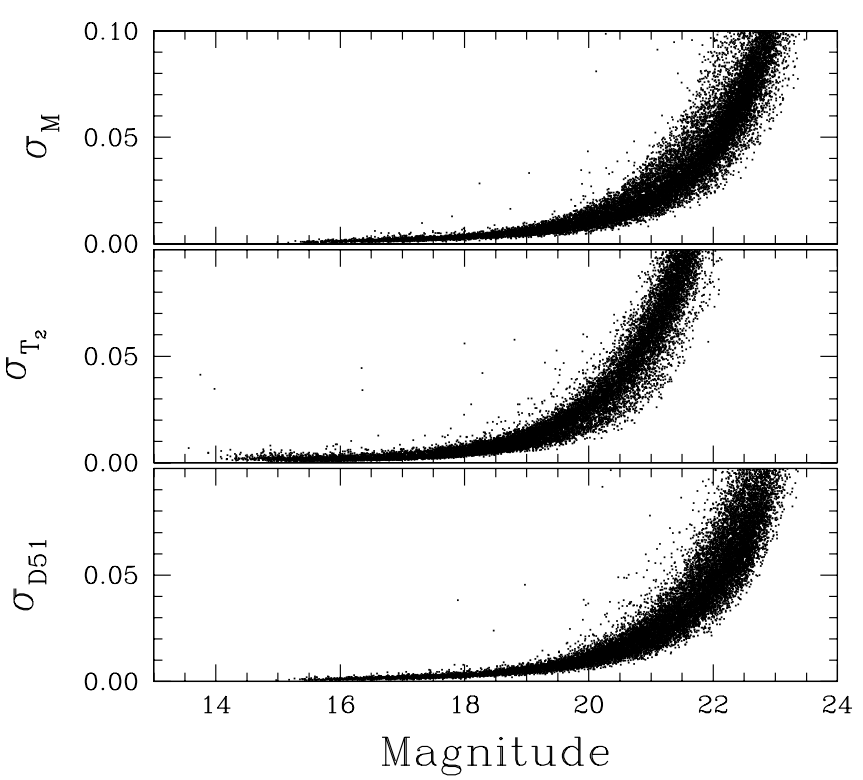

FIG. 1.-DAOPHOT internal errors for stellar objects in our program fields as functions of magnitudes in each band.

our experiments show that the acceptable range changes as a function of radial distance from the center of Leo I because crowding near the center affects the stellar profiles. Therefore, we have elected to use a different $\chi$ selection criteria for sources inside a major-axis radial distance ${ }^{15}$ of $8^{\prime}(\chi<2.5)$ and outside this radius $(\chi<1.3)$.

In Figure 1 we plot the DAOPHOT internal photometric error for the final catalog stars as functions of calibrated magnitudes in the three different bands.

Figure $2 a$ shows the sky distribution of all stars detected by DAOPHOT in celestial coordinates. The varying density of sources reflects differences in limiting magnitude and seeing among our different fields. Figure $2 b$ shows the distribution of sources to a uniform depth. In Figure 3 we show the $\left(M-T_{2}, M\right)_{0}$ and $(M-$ $\left.T_{2}, T_{2}\right)_{0}$ CMDs for the stars in Figure $2 a$. The left and right panels of Figure 3 show the CMDs for stars in the central field of Leo I (C field) and in all other fields, respectively. The dominant CMD structure seen in the left panels is the upper part of the Leo I red giant branch (RGB). The prominent Leo I red giant clump (RGC) is present at $21.5<M_{0}<23.0\left(20.0<T_{2,0}<22.0\right)$ and $0.6<\left(M-T_{2}\right)_{0}<1.6$. A small clump of asymptotic giant branch $(\mathrm{AGB})$ stars at $M_{0} \sim 19.5\left(T_{2,0} \sim 17.5\right)$ and $\left(M-T_{2}\right)_{0} \sim$ 2.0 is also apparent. One other noticeable feature in the left panels of Figure 3 is the group of stars at $0.0<\left(M-T_{2}\right)_{0}<0.7$ and $19.0<M_{0}<21.5\left(19.5<T_{2,0}<21.0\right)$. These stars are thought to be anomalous Cepheids or short-period Cepheids with age of a few hundred Myr (Gallart et al. 1999b). The right panels of Figure 3 are more or less typical CMDs for a high Galactic latitude field containing a mixture of Galactic disk stars, halo giants, field $\mathrm{HB}$ stars, and blue stragglers. The sharp edge at $\left(M-T_{2}\right)_{0} \simeq$ 0.65 is due to the MSTO of field stars smeared in apparent magnitude by the range of distances along the line of sight (see, e.g., Reid \& Majewski 1993; Chen et al. 2001; Siegel et al. 2002). The many stars bluer than $\left(M-T_{2}\right)_{0}=0.65$ are likely field HB stars and blue stragglers. However, some low density of Leo I stars of

\footnotetext{
15 Until we derive new structural parameters of Leo I using our photometric data, we use those of IH95, i.e., $r_{t}=12.6^{\prime}$, P.A. $=79^{\circ}, e=0.21$, for defining the tidal boundary of Leo I.
}
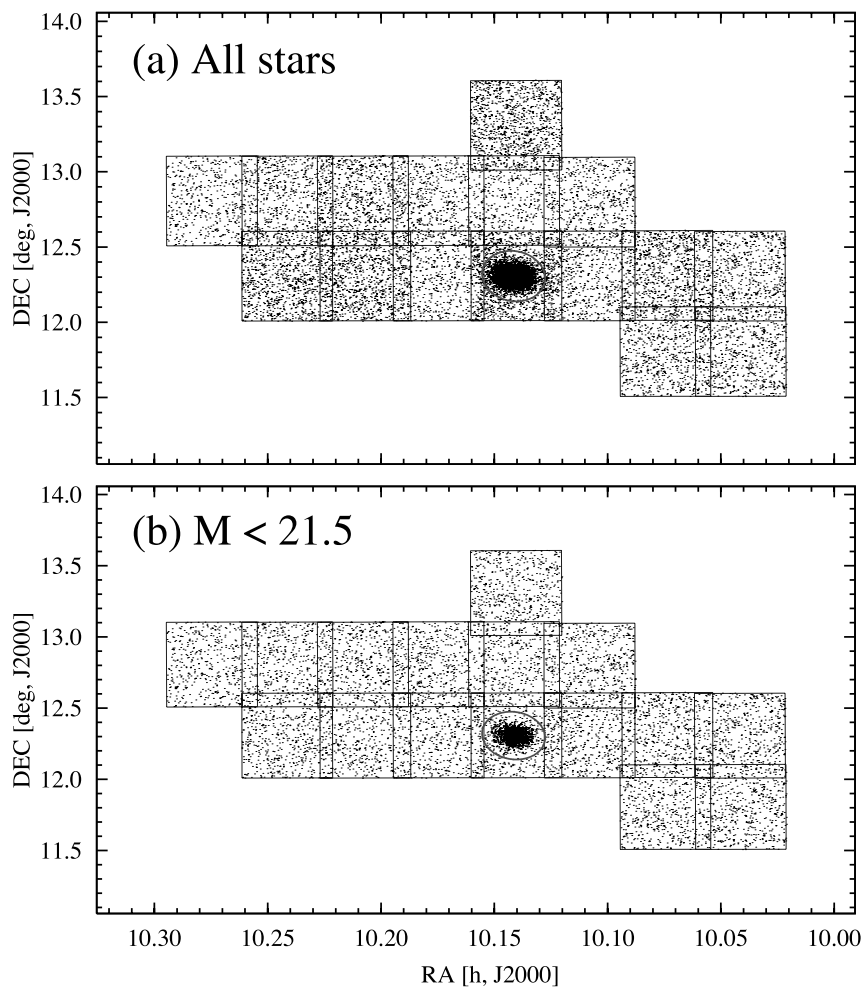

FIG. 2.-Maps of ( $a$ ) all stars detected in our survey and $(b)$ stars brighter than $M=21.5$. The solid lines give rough indication of the boundaries of each observed field. The ellipses represent the Leo I tidal boundary derived by IH95 $\left(r=12.6^{\prime}\right.$, P.A. $\left.=79^{\circ}, e=0.21\right)$, and the asterisks mark the positions of the first-magnitude star Regulus. The inhomogeneities in density among the fields of (a) are a reflection of variation in limiting magnitude across our survey area due to different observational conditions. Note that the inhomogeneities are absent in (b). [See the electronic edition of the Journal for a color version of this figure.]

all types may also lie in the outer fields, swamped by the MW foreground.

\section{IDENTIFICATION OF LEO I GIANT STAR CANDIDATES}

The methodology we use in this study to select clean samples of Leo I giant star candidates is adopted from that used by Majewski et al. (2000b) and subsequent papers in this series. In summary, we photometrically select stars that have (1) magnesium $(\mathrm{Mg} \mathrm{H}+\mathrm{Mg} b)$ band/line strengths consistent with those of giant stars and (2) combinations of surface temperature and apparent magnitude consistent with the RGB of Leo I. In the following sections we demonstrate the applications of these two criteria using our $M, T_{2}$, and DDO51 photometry. To improve the reliability of our Leo I giant star catalog, we restrict our sample to the stars that have photometric errors less than 0.04 mag in each band.

\subsection{Giant Star Discrimination in the Color-Color Diagram}

The DDO51 filter measures the strength of the $\mathrm{Mg} \mathrm{H}+\mathrm{Mg} b$ spectral feature, which is a good indicator of stellar surface gravity. Since dwarf and giant stars are differentiated by their surface gravities, we use the ( $M-$ DDO51) colors (where $M$ filter acts as a continuum measure against DDO51) combined with the surface temperature-sensitive $\left(M-T_{2}\right)$ color $^{16}$ to separate the foreground

\footnotetext{
16 Majewski et al. (2000a) have shown that the $\left(M-T_{2}\right)$ has a linear relationship with $V-I$, which is a good surface temperature indicator for late-type stars.
} 

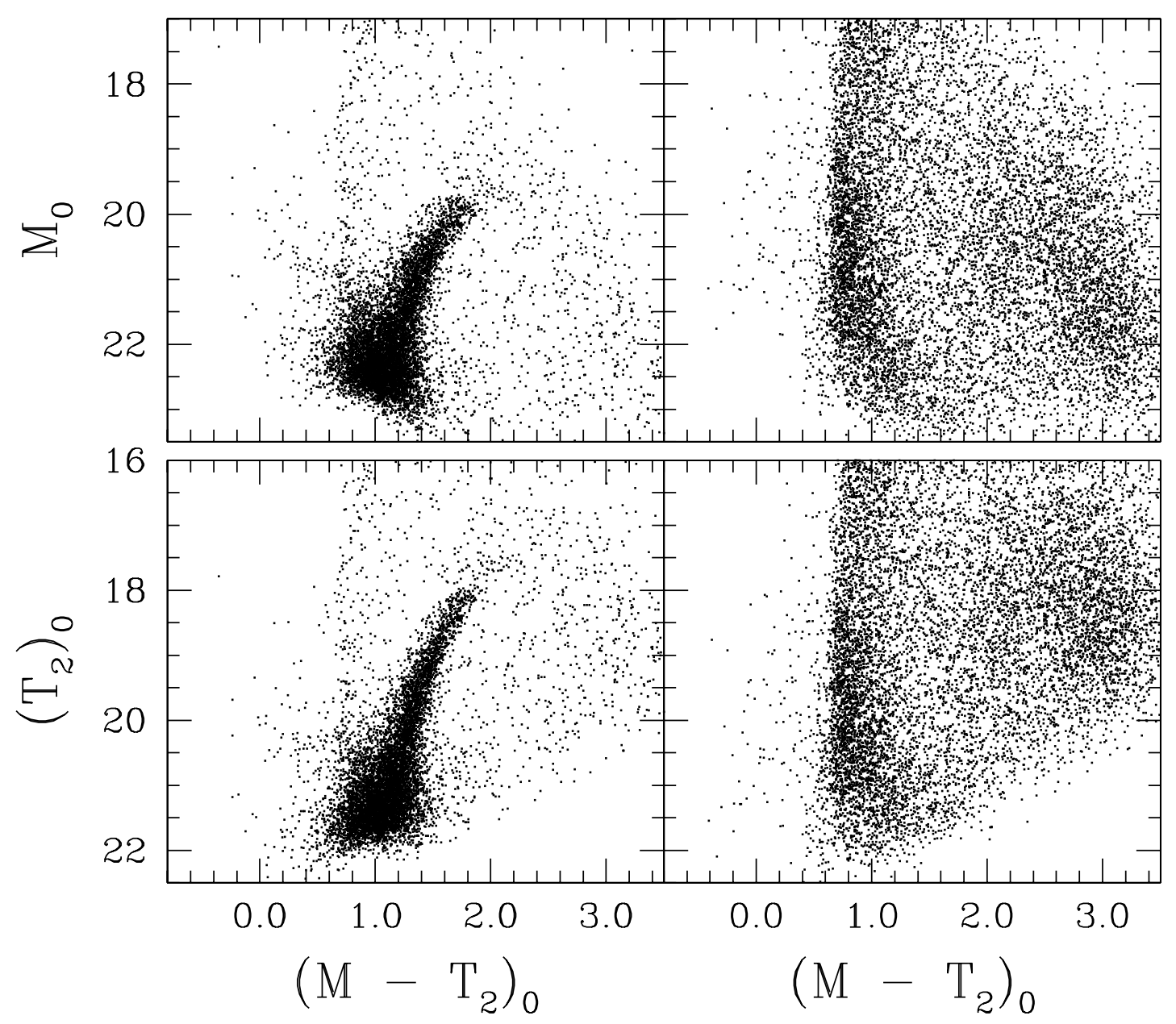

FIG. 3.-Dereddened $\left(M-T_{2}, M\right)_{0}$ and $\left(M-T_{2}, T_{2}\right)_{0}$ CMDs for stars in C frame (left panels) and all other frames (right panels).

dwarf stars and distant metal-poor giant stars. The use of the $\left(M-T_{2}, M-\right.$ DDO51) color-color diagram as a dwarf/giant separating tool has been utilized in several studies (Majewski et al. 2000a, 2000b; Morrison et al. 2000; Palma et al. 2003; Westfall et al. 2006; Muñoz et al. 2005, 2006).

Figure 4 shows the color-color diagrams for all stars that survived the magnitude error, SHARP, and $\chi$ rejection. To better show the difference of the $\left(M-T_{2}, M-\right.$ DDO51) distribution between two distinct components, i.e., Leo I giants and foreground dwarf stars, we divide our plot into stars in $(a)$ the $\mathrm{C}$ field alone and $(b)$ all other fields. The central part of Figure $4 a$ is dominated by Leo I giant stars, whereas Figure $4 b$ shows mainly the elbow-shaped, high-metallicity dwarf star locus and only a few stars in the classic "giant" region of the diagram (see Majewski et al. 2000b). The solid bounding box is defined as follows. The bottom and right boundaries were set by using the distribution of Leo I giant stars in Figure $4 a$. The lower left boundary was drawn roughly parallel to the dwarf locus but offset by about $+0.1 \mathrm{mag}$ in $M$ - DDO51 to account for the increased color errors at the faint end of our data set. We note from Figure $4 a$ that this conservative limit does result in the loss of the bluest Leo I giant stars but ensures that few dwarf stars will scatter into our selection. We truncate the upper left boundary of the box at $\left(M-T_{2}\right)_{0}=1.0$ since this corresponds to the blue boundary of our color-magnitude selection region discussed below. Stars are considered to be giant candidates if they are inside the bounding limits in the two-color diagram. Our color-color selection rejects all but the most metal-poor $([\mathrm{Fe} / \mathrm{H}] \lesssim-2.0)$ foreground dwarf stars, whereas most dwarf stars at higher metallicities lie mainly on the elbow-shaped locus (Paltoglou \& Bell 1994; Majewski et al. 2000a).

\subsection{Identifying Leo I Giant Star Candidates in the Color-Magnitude Diagram}

To select a purer sample of Leo I giants, we now apply a second, color-magnitude criterion to our giant candidate list. Regardless of the angular separation from the core of Leo I, any giant star in Figure 2 associated with Leo I should have a combination of $\left(M-T_{2}\right)$ and $M$ magnitude that places them within the giant branch of Leo I. Figure $5 a$ shows the CMD for giant candidate stars within the IH95 tidal boundary of Leo I, while Figure $5 b$ is for all of the giant candidate stars. We use the stars in Figure $5 a$ to delineate the RGB structure in the CMD. The boundaries were drawn to roughly follow the overall structure of the giant branch. The box is slightly extended to a point brighter and redder than the TRGB to include some Leo I stars on the tip of the AGB. The lower boundary was set at $M_{0}=21.5$ where the luminosity function of our color-color-selected stars turns over due to incompleteness. We note that the nine stars brighter than $M_{0}=21.5$ lying outside of the RGB selection region in Figure $5 a$ are likely other (asymptotic) giant members of Leo I, but we elect to exclude these few stars in the interests of maintaining a conservative selection that excludes interloping nonmembers. This CMD selection criterion is finally applied to the entire sample of giant candidates as shown in Figure $5 b$. In this way, a total of 1282 stars are selected as Leo I giant candidates. 


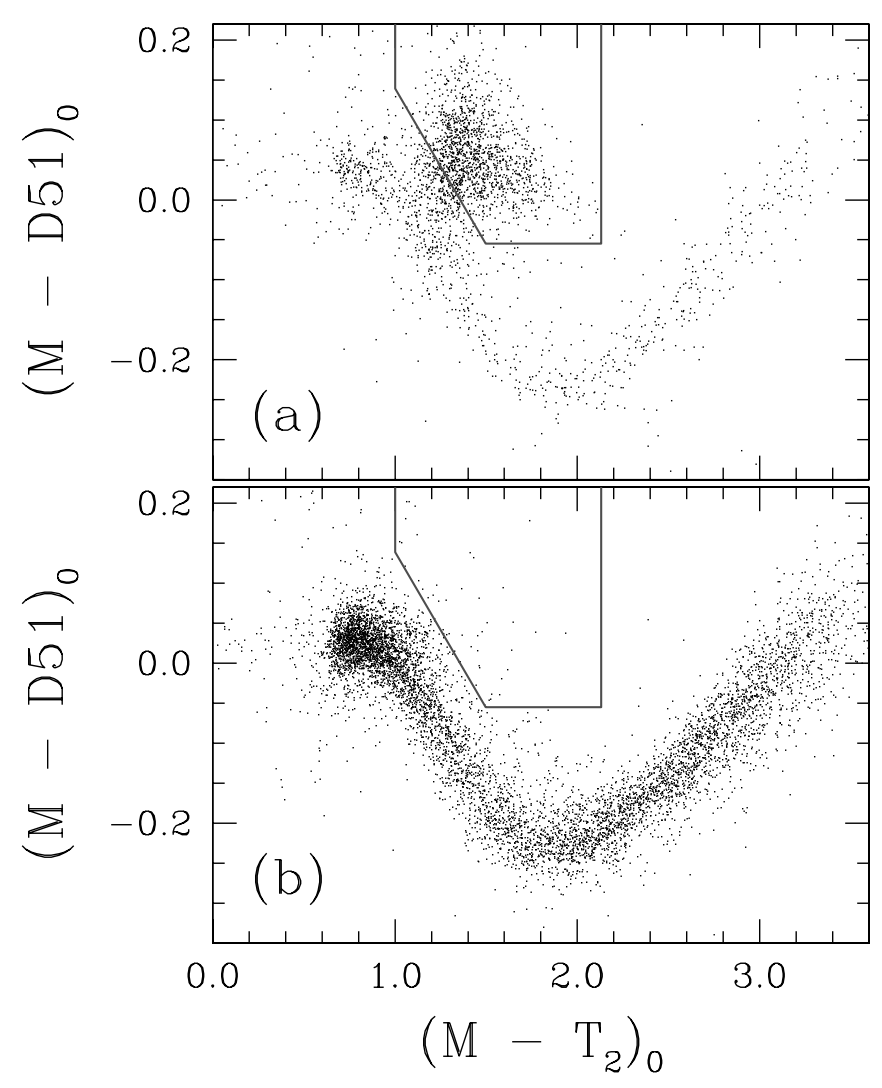

FIG. 4.- $\left(M-T_{2}, M-\text { DDO51 }\right)_{0}$ diagrams for stars in $(a) \mathrm{C}$ frame and $(b)$ all other frames. The box drawn with solid lines shows the bounding region we have employed to select metal-poor giant star candidates. [See the electronic edition of the Journal for a color version of this figure.]

\subsection{Leo I Giant Stars in Earlier Spectroscopic and Photometric Studies}

It is worthwhile to compare the results of our giant star selection criteria against known spectroscopically and photometrically confirmed Leo I members. M98 obtained spectra of 32 stars within the core radius $\left(r_{c}\right)$ of Leo I and another one just outside of $r_{c}$. The results from their derived RVs show that all 33 of these stars in this densest part of Leo I are members of the dSph. There

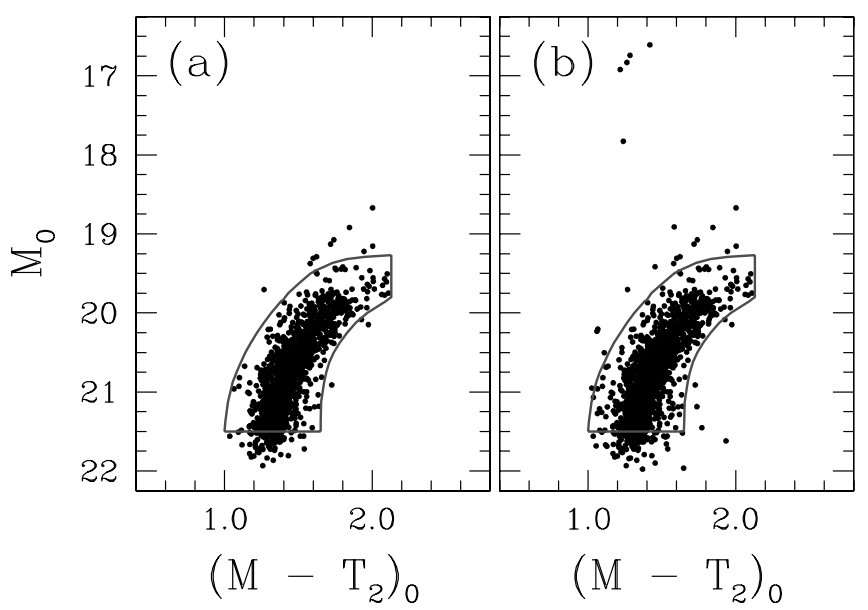

FIG. 5.- $\left(M-T_{2}, M\right)_{0}$ CMD for stars selected as metal-poor giants in Fig. 4 (a) within the tidal boundary derived by $\mathrm{IH} 95$ and $(b)$ within our entire survey area. The bounding boxes shown by the solid lines are our CMD selection criteria. [See the electronic edition of the Journal for a color version of this figure.]

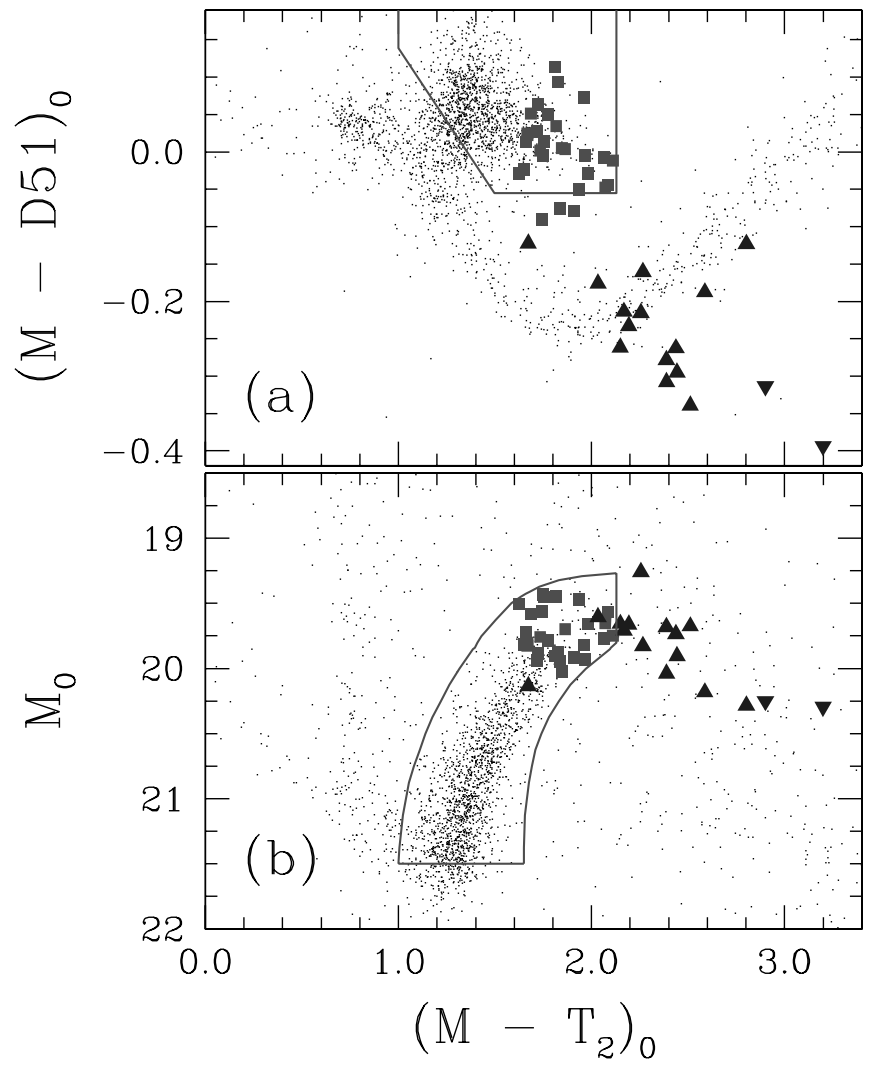

FIG. 6.-Positions of the previously identified Leo I giant stars shown along with our catalog of stars with photometric errors less than 0.04 in the C field: $(a)$ in the color-color diagram, and $(b)$ in the CMD. The filled squares are for red giants and asymptotic giants spectroscopically observed by M98. The filled triangles are for carbon stars spectroscopically confirmed by Azzopardi et al. (1985, 1986), and the inverted filled triangles are for carbon stars photometrically identified by Demers \& Battinelli (2002). [See the electronic edition of the Journal for a color version of this figure.]

have also been carbon star surveys of Leo I using spectroscopic (Azzopardi et al. 1985, 1986) and photometric data (Demers \& Battinelli 2002). Stars 21 and 24 of the M98 sample are the same stars listed as carbon stars Azzopardi18 and Azzopardi1, respectively, in Azzopardi et al. (1985, 1986). We have cross identified 28 red giant stars of M98, 14 carbon stars of Azzopardi et al. (1985, 1986), and 2 carbon stars of Demers \& Battinelli (2002) with our photometry. Other stars in the past literature that were not cross identified in our survey are missing because they are situated within the physical gaps of the Mosaic CCD chips.

Figure 6 shows the identified giant (filled squares) and carbon stars (filled triangles) in the color-color diagram (Fig. 6a) and the CMD (Fig. 6b). The two reddest carbon stars (inverted filled triangles) are from Demers \& Battinelli (2002). It is immediately apparent that the carbon stars are not situated within the giant star selection region in the color-color diagram. Instead, these stars extend to the lower right part of the diagram, indicating that the carbon stars have strong absorption features within the DDO51 filter bandwidth. In fact, carbon stars are well known to have a strong $\mathrm{C}_{2}$ Swan band absorption feature at a band head of $5165 \AA$ (for a representative spectrum of an R-type carbon star see, e.g., Fig. 1 of Christlieb et al. 2001), well within the bandwidth of the DDO51 filter. Despite the weak surface gravity of these cool giant stars, the strong $\mathrm{C}_{2}$ absorption lines dominate the $M-$ DDO51 color and place the carbon stars away from the giant selection regions shown in Figure 6. On the other hand, all but three of the normal giant stars in the M98 sample are within the color-color 


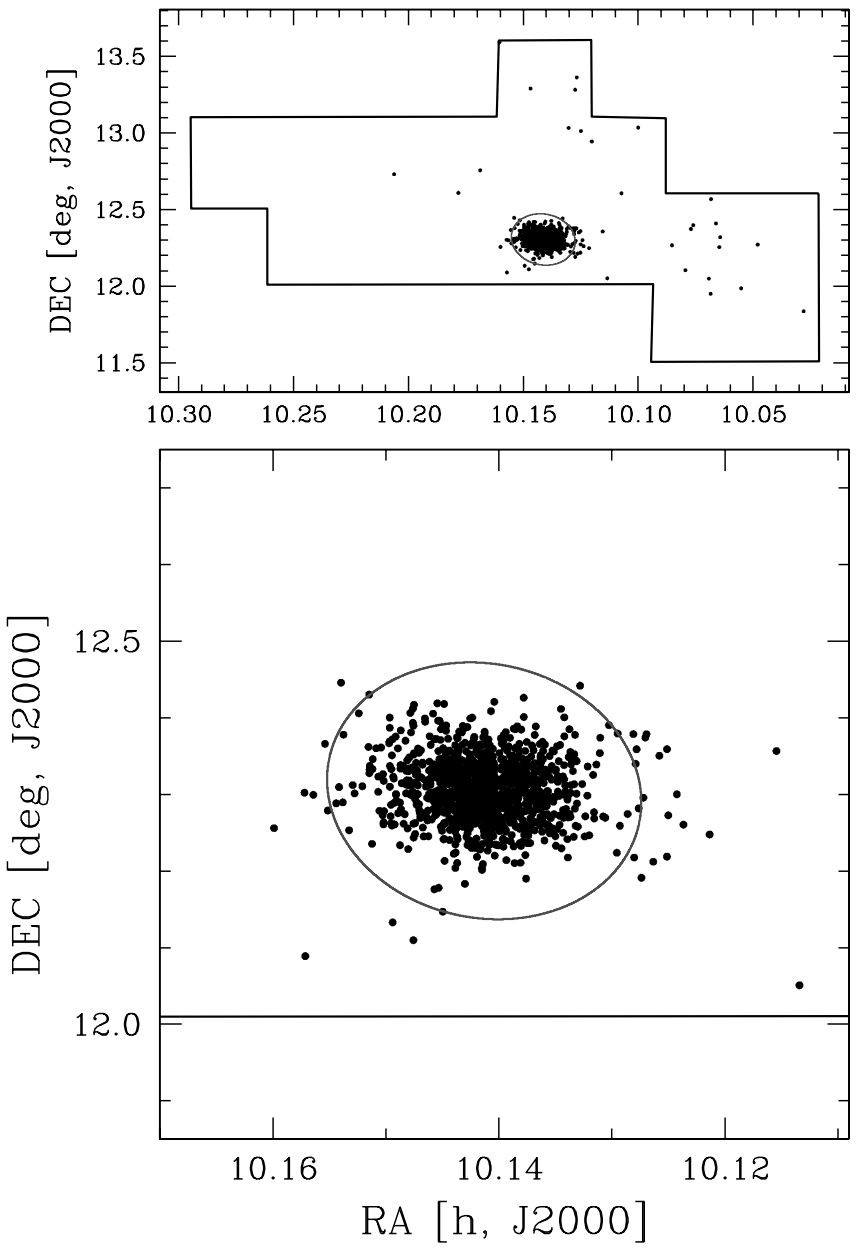

FIG. 7.-Distribution on the sky of stars selected as "Leo I-like" giants by our color-color and color-magnitude selection methods. The bottom panel is a blowup of the central part in the top panel. The region enclosed by the solid lines is our survey area. The ellipse shows the tidal boundary derived by IH95, and the hatched region in the bottom panel is outside of our survey area. [See the electronic edition of the Journal for a color version of this figure.]

selection box (Fig. 6a). We could include these three stars by extending the lower boundary of our color-color selection box, but because it might introduce more contaminants (such as metal-poor subdwarfs from the thick disk or halo), we opted not to do so. Our RGB selection in Figure $6 b$ includes all M98 giant stars. The placement of filled squares in Figure 6 thus substantiates the reliability of our selection technique, although we note that all cross-identified noncarbon giant stars of Leo I lie in the bright end of the giant branch where photometric errors are on average only $0.01 \mathrm{mag}$ in all three bands. However, in $\S 5$ we present new spectroscopy showing that the catalog maintains its reliability to significantly fainter magnitudes.

\subsection{Spatial Distribution of Leo I Giant Star Candidates}

Figure 7 shows the sky distribution of all stars selected as Leo I RGB stars by our color-color and color-magnitude selection methods. Of particular interest are the 52 stars that lie outside the limiting radius found by $\mathrm{IH} 95$ and 26 stars outside twice the IH95 limiting radius. The inner part of the spatial distribution (bottom panel) shows more clearly the falloff in the concentration of Leo I giant candidates. Interestingly, we find that stars spill over the IH95 boundary from the main body of Leo I predominantly along the semimajor axis to both sides. We dis-
TABLE 2

Number of Color-Color-selected Giants in RGB Bounding BoXes Offset along Brighter Magnitudes

\begin{tabular}{|c|c|}
\hline$\Delta M$ & Number of Giants \\
\hline 0.0 & $1196^{\mathrm{a}}$ \\
\hline 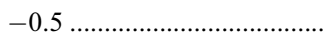 & $709^{\mathrm{a}}$ \\
\hline 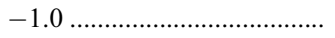 & $253^{\mathrm{a}}$ \\
\hline 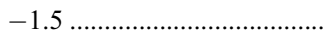 & $27^{\mathrm{a}}$ \\
\hline 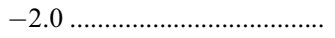 & 5 \\
\hline 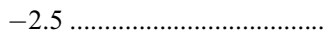 & 2 \\
\hline 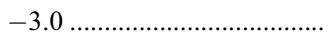 & 1 \\
\hline 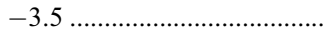 & 5 \\
\hline-4.0 & 4 \\
\hline-4.5 ..................................... & 4 \\
\hline-5.0 & $0^{\mathrm{b}}$ \\
\hline 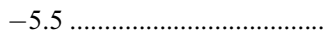 & $0^{\mathrm{b}}$ \\
\hline
\end{tabular}

a Sample contains stars from the Leo I system.

b Sample has an incomplete sampling of the CMD at the bright end.

cuss the more extended distribution of "Leo I giant candidates" below.

\subsection{Evaluation of Background Level}

The two-criterion Leo I giant star selection method described above is designed to maximize the reliability of our candidate selection. However, our final sample may still contain contaminants. Any halo giant or extreme $([\mathrm{Fe} / \mathrm{H}]<-2.0)$ subdwarf with an observed $\left(M-T_{2}, M\right)_{0}$ combination similar to our Leo I RGB stars can be caught in our color-color and color-magnitude selection net. So too can normal metallicity dwarfs artificially scattered into the selection from photometric errors (these will be MSTO dwarfs or subgiants with $M_{V} \lesssim 6$, so that these will also be predominantly from the MW halo). The number of these contaminants can be estimated by a simple technique described in Majewski et al. (2000b) and Palma et al. (2003): offset the RGB selection box to brighter magnitudes and count the stars that fall in the offset box. Assuming that the density of halo stars is approximately represented by an $R^{-3}$ power law (e.g., Siegel et al. 2002), the number of halo stars per unit solid angle is flat with respect to magnitude to first order. Therefore, the number of stars within the offset box should remain roughly constant as a function of magnitude offset. The Carina and Ursa Minor fields were in compliance with such predictions (Majewski et al. 2000a; Palma et al. 2003). However, inspection of Figure $5 b$ already suggests that the amount of contamination in our case is very low because there are very few stars picked as giant candidates that are brighter than the RGB selection box. Nevertheless, we repeated the exercise and the results are presented in Table 2 . The four faintest offset bins are still counting a number of Leo I giants including the AGB stars left out by our color-magnitude selection box. At the largest magnitude offsets, the sampling of the CMD is incomplete due to the saturation of bright stars in our fields. Therefore, the bins to be considered are $-5.0<\Delta M \leq-2.0$. We take the average of these six bins and estimate the background level in our case to be $3.5 \pm 1.9$ for the entire field and $3.4 \pm 1.8$ for the field outside an ellipse drawn from the IH95 structural parameters, which has an area of $\sim 0.11 \mathrm{deg}^{2}$ (errors have been calculated assuming Poissonian statistics). This converts to a background density of $0.8 \pm 0.4 \mathrm{deg}^{-2}$. The number of Leo I giant candidates found outside the King limiting radius is 20 times this background level. While our derived background level is considerably smaller than was found in the previous studies of the Carina and Ursa 
Minor fields, Leo I is at a higher Galactic latitude and, moreover, we have employed a very conservative color-color selection. For example, if we use a different color-color selection box by bringing the lower left boundary closer to the metal-rich dwarf locus than the one used before by $\Delta(M-$ DDO51) $=0.05$, the background density becomes $12.5 \pm 3.5 \mathrm{deg}^{-2}$. Typically, the photometric errors are larger at fainter magnitudes. Since fainter Leo I RGB stars are bluer and closer to the dividing line between the giant and dwarf locus in the color-color diagram, the likelihood of dwarfs contaminating our giant selection is greater at fainter magnitudes. Our very conservative selection of Leo I giant candidates gives us confidence that residual contamination will be very low.

The low contamination level of our Leo I giant candidate list is illustrated by the top panel of Figure 7: no Leo I giant candidates exist in the large area east of R.A. $=10.22^{\mathrm{h}}$, which suggests that the zero-level background is reached within our survey region. But this begs the question of the origin of the numerous, very widely separated Leo I giant candidates in the western half of our survey area. There are 26 candidates outside of twice the King limiting radius in our survey area (all but four of them to the west of Leo I's center), but the background level derived above suggests that only about 3-4 of these stars should be contaminants. Until we can obtain spectroscopy of these stars, it is difficult to assess whether they are part of Leo I (as tidal debris), they are MW contaminants, or some combination of both. Tidal disruption models (e.g., $\S$ 6.1.2) predict that stars released during the latest perigalactic passage are mostly spread out along the major axis of the satellite, and this could account for at least half of these spatial outliers. Moreover, stars that became unbound during older perigalactic passages tend to be more irregularly distributed around the satellite (see Fig. 22 below). It is therefore possible that many of the Leo I giant candidates located far out from the main body could very well be unbound stars of the latter type. Certainly the large-scale inhomogeneity of the distribution is highly suggestive of substructure of some kind. Nevertheless, because of the present ambiguous nature of these widely separated stars, we have deliberately and conservatively elected to confine the remaining analyses in this paper to the central square degree around Leo I.

\section{STRUCTURE OF LEO I}

In this section we present the results from profile fitting the two-dimensional distribution of Leo I giant candidates. A massto-light ratio of the bound population is calculated via the new set of structural parameters using the standard techniques of King (1966) and Illingworth (1976). We comment on newly identified, potential extratidal Leo I stars and also present isodensity contour plots of the Leo I system.

\subsection{Profile Fitting and Structural Parameters}

Structural parameters for Leo I were first derived by Hodge $(1963,1971)$ and later by IH95. All three studies used King profiles to fit their photographic observations. We use the positions on the sky of our giant candidates to explore the radial density profiles. To do so, we fit the surface density distribution of our Leo I giant candidates using two different models: the singlecomponent King model and a power law plus core (PLC). The fittings were done using a combination of Bayesian and maximum likelihood techniques similar to that employed by Kleyna et al. (1998). Errors for each parameter were estimated using a Bayesian approach in conjunction with a Markov Chain technique. The details of the fitting and error-estimating algorithms are described in Ostheimer (2002). A single-component King model has been widely used to fit many dSph galaxies (e.g., IH95). The PLC model was adopted by Kleyna et al. (1998) to fit their
Ursa Minor surface density profile. We describe our fitting method below in detail.

Several dSphs are found to have outer profile "breaks" (i.e., slope changes) from their central King profile density distributions: Fornax and Sculptor (Eskridge 1988a, 1988b; Westfall et al. 2006), Carina (Majewski et al. 2000b, 2005; Muñoz et al. 2006), Ursa Minor (Palma et al. 2003), and Sgr (Majewski et al. 2003). Although statistically insignificant, IH95 found an excess of stars with respect to their King fit in the outer region of Leo I. An excess of stars should show up in our radial density profile based on the appearance of stars spilled over from the main body of Leo I along the east/west in Figure 7. Since a fit to a distribution of stars including the excess of stars in the outer regions will likely inflate the derived core and limiting radii, we have taken the following steps to find a new King profile fit: First, we limit the stars used to derive the structural parameters to those within $\sim 13^{\prime}$ from the center of Leo I in the east/west direction. Next, six structural parameters (right ascension and declination of the dSph center, position angle, core radius, ellipticity, and limiting radius) are derived from this stellar sample. Finally, the background level derived in $\S 3.5$ is combined with the six parameters to represent a newly fitted single-component King model. A similar approach was taken in the study of the Sgr profile by Majewski et al. (2003). The PLC model, on the other hand, was fitted using the entire data set in one pass with the background level set to the same value used for fitting the King model.

Table 3 lists the best-fit parameters and errors for each profile. The first two lines in the table are for the new King and PLC profile fits. For comparison, we also list the King profile parameters derived in earlier Leo I studies by Hodge $(1963,1971)$ and IH95. The newly derived center of Leo I is at $(\alpha, \delta)_{\mathrm{J} 2000.0}=$ $\left(10^{\mathrm{h}} 08^{\mathrm{m}} 28.68^{\mathrm{s}},+12^{\circ} 18^{\prime} 19.7^{\prime \prime}\right)$.

Our derived core radius and ellipticity for either the PLC or King profile fit are larger than those derived by IH95 but are much closer to those of Hodge (1971). Similarly, our limiting radius is in better agreement with that of Hodge (1971) although IH95's limiting radius is also consistent within the errors. To illustrate how our new structural parameters better fit the sky distribution of Leo I giant candidates, the new King limiting radius is plotted over the sky distribution of Leo I giant candidates in Figure 8 (compare to IH95 limiting radius size and shape in Fig. 7). Figure 8 also shows the ellipse corresponding to the break radius ( $r_{\text {break }}$; see below for the determination of the break radius).

With the newly derived Leo I structural parameters, we construct radial density profiles by calculating the number density of Leo I giant candidates in elliptical annuli of varying major-axis radial size. Although our coverage has small gaps because of spaces between the Mosaic CCD chips, our profile fitting program interpolates over them when doing any relevant calculation. Figure $9 a$ shows the King model with structural parameters derived by IH95 overlaid on the giant candidate radial profile. Figure $9 b$ shows our new King model, and Figure $9 c$ shows the PLC profile fit.

We now concentrate on the newly derived King model (Fig. 9b). The observed density profile clearly departs from the model beyond $r_{\text {maj }} \sim 10$, and this "break population" dominates the density out to the entire survey range even when uncertainties in both counting and background estimation are taken into account. Such profile breaks are also seen in $N$-body models of tidally disrupting satellite galaxies (Johnston et al. 1999, 2002). In these models, the density profiles of the break populations typically assume power-law shapes, $\Sigma_{N} \propto r^{-\gamma}$. In Figure $9 b$ we overplot power laws with $\gamma=2,3,4$, and 5 in the range $r_{\text {maj }}>$ $10^{\prime}$. As seen from the figure, our observed profile beyond the 
TABLE 3

Fit Parameters for Leo I

\begin{tabular}{|c|c|c|c|c|c|}
\hline Reference & $\begin{array}{c}r_{c} \\
\text { (arcmin) }\end{array}$ & $\begin{array}{c}r_{t} \\
\text { (arcmin) }\end{array}$ & $\begin{array}{c}\epsilon \\
(=1-b / a)\end{array}$ & $\begin{array}{l}\text { P.A. }^{a} \\
(\mathrm{deg})\end{array}$ & $\nu^{\mathrm{b}}$ \\
\hline 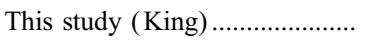 & $5.4 \pm 0.4$ & $13.4 \pm 0.7$ & $0.37 \pm 0.02$ & $84 \pm 2$ & \\
\hline This study (PLC) & $7.3 \pm 0.8$ & $\ldots$ & $0.37 \pm 0.02$ & $85 \pm 2$ & $4.8 \pm 0.6$ \\
\hline 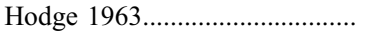 & $\ldots$ & $14.3 \pm 1.0$ & $0.31 \pm 0.07$ & $\ldots$ & $\ldots$ \\
\hline 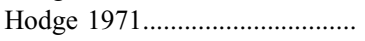 & 4.5 & $13.9 \pm 0.5$ & $0.31 \pm 0.07$ & $\ldots$ & $\cdots$ \\
\hline IH95 & $3.3 \pm 0.3$ & $12.6 \pm 1.5$ & $0.21 \pm 0.03$ & $79 \pm 3$ & $\ldots$ \\
\hline
\end{tabular}

${ }^{\text {a }}$ Major-axis position angle measuring from north $=0^{\circ}$ to east $=90^{\circ}$.

${ }^{\mathrm{b}}$ See eq. (1) of Kleyna et al. (1998).

break point is not fitted well by a single power law, but instead the first three radial bins starting at and including the break radius follow a power law with $\gamma=3$, while the next three radial bins follow a power-law density trend with $\gamma=5$. Overall, a power law with $\gamma \sim 4$ best describes the surface density from the break radius to $r_{\text {maj }} \sim 35^{\prime}$. It is worth noting that even for the PLC fit (Fig. 9c), the observed density profile starts to depart from the model at $r_{\text {maj }} \sim 10^{\prime}$. This indicates that the existence of the break population does not strongly depend on the choice of fitting models.

The surface density profile of Figure $9 b$ roughly remains flat in the range $35^{\prime} \lesssim r_{\text {maj }} \lesssim 80^{\prime}$ and then drops to the background level beyond that. Such a flat density distribution at large radii has not been seen in the density profiles of other $\mathrm{dSph}$ galaxies. In $\S 6.1 .1$ we hypothesize that the complex form of the density profile might be attributable to specific patterns in the Leo I massloss history.

IH95 found that Fornax, Sculptor, and Ursa Minor show asymmetric residual structure after a best-fitting, smooth elliptical profile has been subtracted from their two-dimensional distribution. Figure 8 gives some impression of an east-west inhomogeneity in the distribution of Leo I stars. To check this, we plot in Figure 10 the radial density profiles for the east and west halves of the gal-

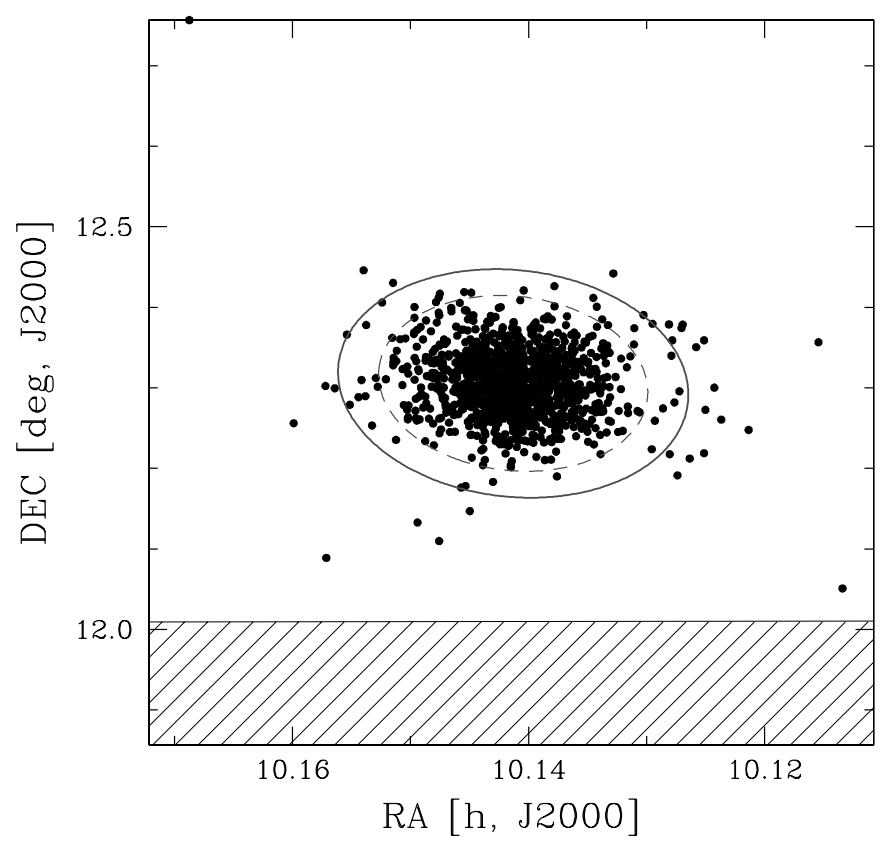

FIG. 8. - Sky distribution of Leo I giant candidates with the new King limiting radius (solid line) derived in this study. The inner dashed ellipse corresponds to the point where the radial density profile starts to deviate from a single-component King model ( $\S 4.1)$. The hatched region is outside of our survey area. [See the electronic edition of the Journal for a color version of this figure.] axy, separately. In the inner five bins, the western half has slightly higher densities, but at low statistical significance. The densities are then nearly identical out to the second break. For the next four bins after the second break, the western half is again higher in density, and this corresponds to the excess stars to the west, just outside the limiting radius. Nevertheless, the differences are within the Poissonian errors, and we conclude that the structural difference between the eastern and western halves of Leo I is of low significance. However, the fact that the breaks are seen independently in both east and west samples reinforces the reality of these structural features.

\subsection{Mass-to-Light Ratio from Core Fitting}

If we use the King profile parameters in Table 3 to represent the bound part of Leo I, the bound mass can be estimated under

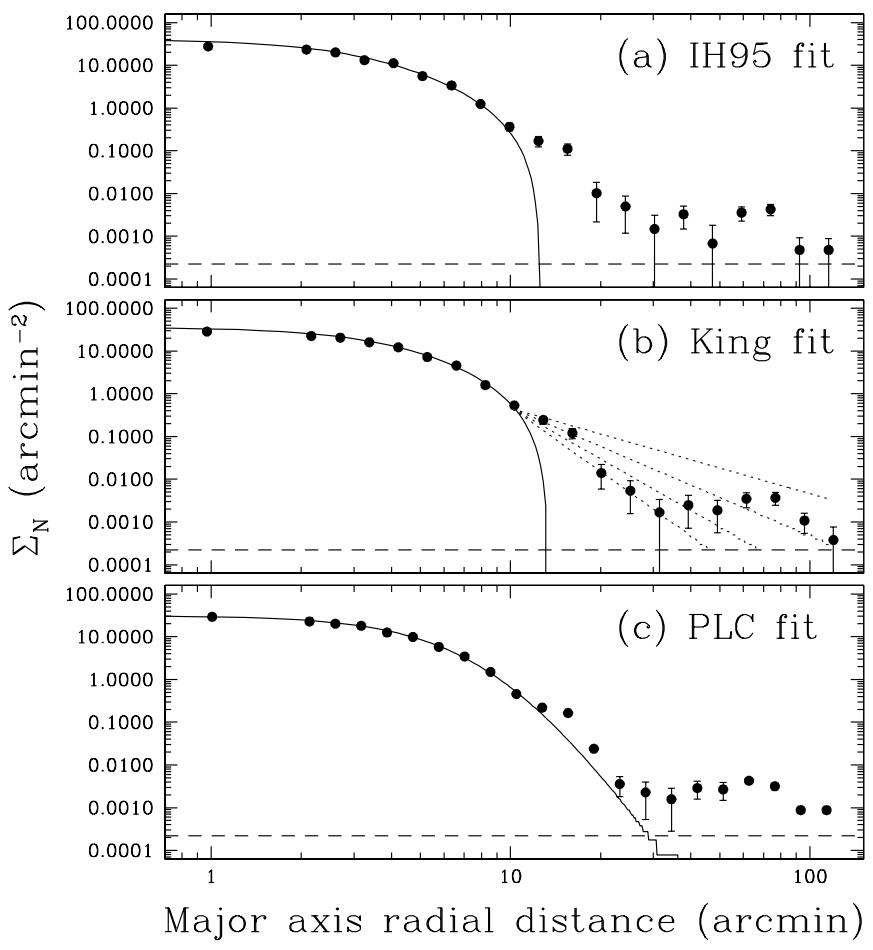

FIG. 9.-Fits of the stellar distribution of Leo I using $(a)$ a King model with parameters derived by IH95, (b) our new King model with a power law, and (c) a power law with core (PLC) model. The horizontal dashed lines are the derived levels of the backgrounds, which were subtracted from the data and the model curves shown. The data points in each panel have slightly different positions because the ellipticities and position angles derived from the fits require different binning of the data points. The dotted lines in $(b)$ show power laws of the form $\Sigma_{N} \propto r^{-\gamma}$, where $\gamma=2.0,3.0,4.0$, and 5.0 ( from top to bottom). The errors for each point include both $1 \sigma$ Poissonian and background estimation errors. [See the electronic edition of the Journal for a color version of this figure.] 


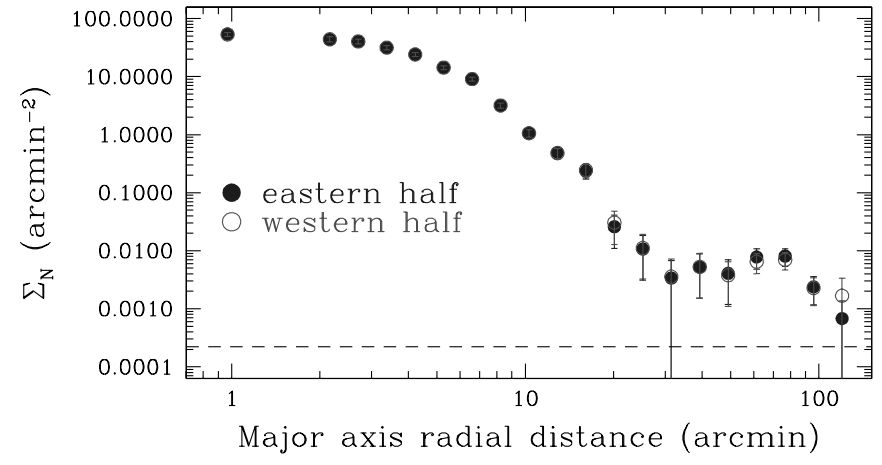

FIG. 10.- Radial surface density profiles for the eastern and western halves of Leo I. The filled and open circles are for eastern and western bins, respectively. [See the electronic edition of the Journal for a color version of this figure.]

the usual assumptions of virial equilibrium and the standard equation given by Illingworth (1976):

$$
M_{\mathrm{tot}}=\frac{166.5 R_{c, g} \mu}{\beta},
$$

where $R_{c, g}$ is the geometric mean of the King core radius $\left[=r_{c}(1-\epsilon)^{1 / 2}\right]$ in parsecs $(271 \pm 26 \mathrm{pc}$ for Leo I $), \mu$ is the King (1966) mass parameter, and $\beta$ is a velocity parameter that is related to the observed velocity dispersion. Both $\mu$ and $\beta$ are strongly dependent on the concentration of a system (King 1962). From the results in Table 3, the concentration of Leo I is $\log \left(r_{t} / r_{c}\right)=0.39 \pm 0.04$. The scaling parameter $\mu$ was taken from an extrapolation of Table 2 of King (1966) and $\beta$ from Figures 4-11 of Binney \& Tremaine (1987). The central velocity dispersion of $\sigma_{0}=8.8 \pm 1.3 \mathrm{~km} \mathrm{~s}^{-1}$ (M98) yields $M_{\mathrm{tot}}=(4.0 \pm$ $1.2) \times 10^{7} M_{\odot}{ }^{17}$ To obtain the corresponding luminosity, we integrate our best-fit model King profile for giant star candidates and scale it to the total luminosity using a central surface brightness of

\footnotetext{
17 Our own derived central velocity dispersion of $8.2 \mathrm{~km} \mathrm{~s}^{-1}$ (see $\S 5.5$ ) only lowers the estimated Leo I mass by $14 \%$.
}

$\mu_{0, V}=22.4$ mag $\operatorname{arcsec}^{-2}$ taken from Table 4 of M98; this yields $L_{\text {tot }}=7.6 \times 10^{6} L_{\odot}$. The $M / L$ using this technique thus becomes $(M / L)_{\text {tot }, V}=5.3 \pm 1.6 M_{\odot} L_{\odot, V}^{-1}$, where the error is from the uncertainty in the mass derived above. Although we have used different structural parameters, we obtain nearly the same $M / L$ as M98. We note that this $M / L$ is actually not that large compared to those typically found for $\mathrm{dSphs}$ and is fairly similar to that of $\mathrm{dE}$ galaxies and globular clusters of similar luminosity. As has been previously found (M98), Leo I has one of the lowest $M / L$ among the Galactic satellites, although one that, by this method, still suggests a significant amount of DM.

\subsection{Isodensity Contours}

In Figure 11 we present the isodensity contour map of Leo I. This map was constructed using the Leo I giant candidates in the following manner: The equatorial coordinates of each star were first converted to a flat Cartesian system via tangential projection centered at the newly derived system center. The Cartesian space was divided into a large grid at intervals of $2.7^{\prime}$ in each dimension. We then counted the number of stars in each grid interval to obtain the density for that part of the map. The gaps between the chips of the Mosaic CCD camera are 50 CCD pixels in the rows and 35 CCD pixels in the columns, corresponding to $\sim 13^{\prime \prime}$ and $\sim 9^{\prime \prime}$ in the sky, respectively. In order to check whether the absence of stars in the CCD gaps produces any artifacts in Figure $11 a$, we performed checks on our data as follows: First, we divided the CCD gaps into segments of $6^{\prime}$ bins and filled each bin with randomly placed artificial stars. The number of stars that went into each bin was calculated by taking the average of the number of Leo I stars on either side of the gaps in similar right ascension/ declination ranges. Once the gaps were filled, an isodensity contour plot was constructed using the exact same procedure as described above. We have also placed artificial gaps with the same size as the real ones in random places and constructed several contour plots. Only slight differences were found between the isodensity contours constructed in these ways and Figure $11 a$. This ensures us that the influence of the CCD gaps on Figure $11 a$ is negligible.
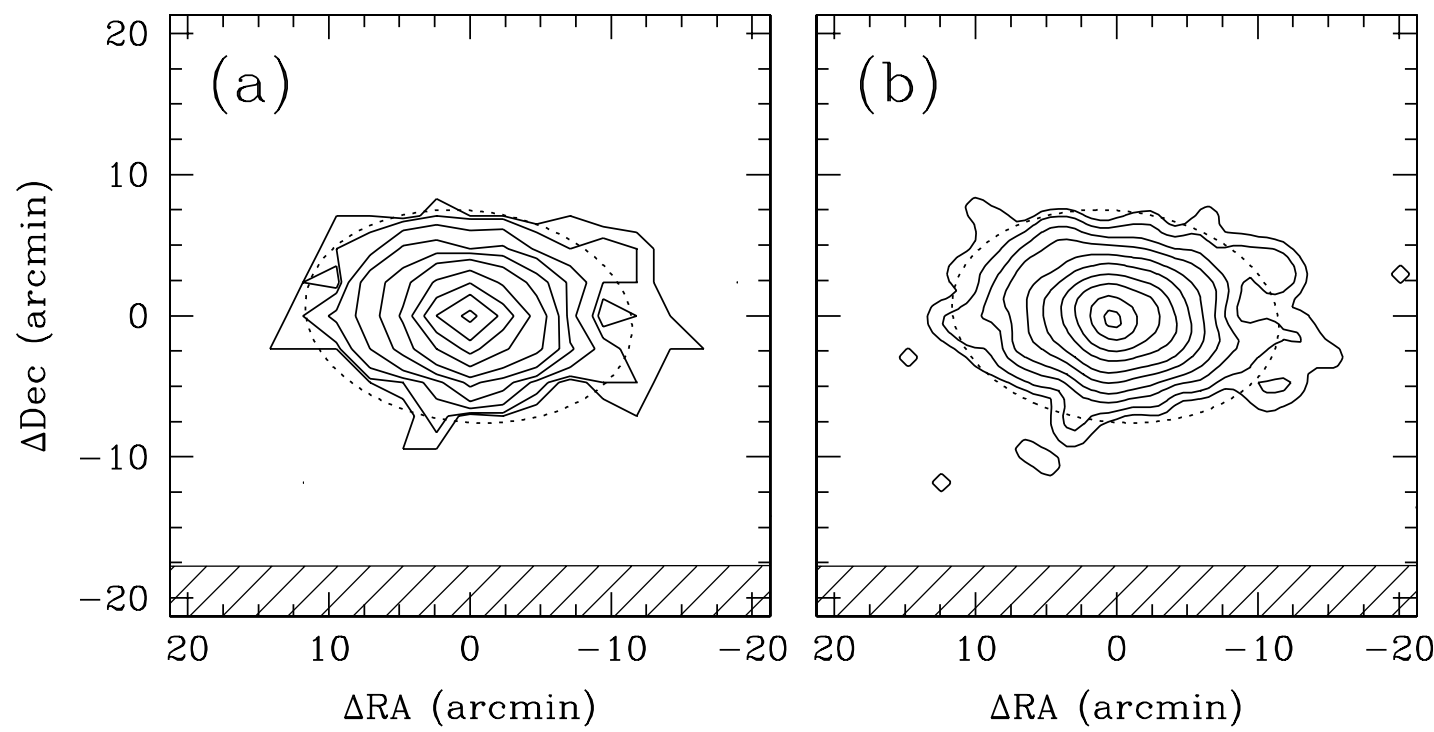

FIG. 11. - (a) Isodensity contour plots of Leo I constructed using Leo I giant candidates. This figure shows the $43 \times 43 \operatorname{arcmin}^{2}$ region centered on Leo I. The newly derived center is at $(\Delta \alpha, \Delta \delta)=(0,0)$. The hatched region is outside of our survey region. The contour levels are $1,2,5,10,20,35,60,90,120$, and 160 stars pixel $^{-1}$. (b) Smoothed isodensity contour plot of Leo I using the same technique for making (a) but with each star represented by a 6.0' wide two-dimensional Gaussian (see $\S 4.3$ ). Each pixel has a dimension of roughly $0.7^{\prime} \times 0.7^{\prime}$. The contour levels are $0.8,2,5,10,20,35,60,90,130$, and $160 \mathrm{pixel}^{-1}$. 
In order to reduce the granularity from the gridding process in our isodensity contour, we constructed a grid 4 times finer than the one used above and replaced each star with a $6.0 \times 6.0 \mathrm{arcmin}^{2}$ grid filled with a two-dimensional Gaussian template $\left(\sigma=1.1^{\prime}\right.$; amplitude $=1$ ). We assigned density to each pixel by counting up the numbers in a similar manner discussed above. The resulting smoothed image is shown in Figure $11 b$.

Overall, the inner contours of Leo I are similar to the isopleth map shown in IH95 (see their Fig. 1d). However, the spillover of stars at the profile break radius is evident in the outer contours of Figure 11 as contour extensions stretching out from the main body along the semimajor axis. It is important to note that the spatial density for the outermost contour in Figure 11 $a$ is still higher than 600 times the background level derived in $\S 3.5$.

\section{SPECTROSCOPY OF LEO I GIANT CANDIDATES}

\subsection{Observations and Basic Reductions}

We obtained spectra of a total of 135 stars in the Leo I field: 49 stars lying just inside and outside the King limiting radius to the west of the Leo I center (field KD1), and 86 stars near the center and along the east major axis of Leo I (field KD2). The goals of our spectroscopic observations are (1) to check further the reliability of our color-color and color-magnitude selections discussed in $\S 3,(2)$ to verify the reality of the break population seen in the radial density profiles of Leo I (see Fig. 9), and (3) to study the dynamics of both the inner and outer parts of the Leo I dwarf.

The spectra were obtained with observations of two multislit masks with the Deep Imaging Multi-Object Spectrograph (DEIMOS; Faber et al. 2003) on the nights of UT 2003 October 29 (field KD1) and 2004 October 15-18 (field KD2) using the Keck II 10 m telescope (see Fig. 16 below for the placement of our masks on the sky with respect to the Leo I giant candidates). The masks were designed using coordinates from our photometric catalog, which have been locked to the astrometric system of the USNO-A2.0 catalog (see $\S 2$ ). Priority for slit selection was given to stars selected to be Leo I giant candidates by the criteria described in $\S 3$. Even for this priority sample, all stars falling within the $5^{\prime} \times 16^{\prime}$ slit mask area of field KD1 could not be observed because of slit overlap. On the other hand, occasional gaps in mask area remained, and these were filled with slits for, in priority order, (1) stars selected as giants, but not as Leo I giants, and (2) any other star in the field without regard to classification. Observations of stars classified as dwarfs prove useful as checks on the reliability of the dwarf/giant separation. The spectrograph was configured with the 1200 line $\mathrm{mm}^{-1}$ grating and $1^{\prime \prime}$ slits with the central wavelength set at $7800 \AA$. This instrumental setup provides $0.33 \AA$ pixel $^{-1}$ dispersion, a spectral resolution FWHM of $1.95 \AA$ ( $68 \mathrm{~km} \mathrm{~s}^{-1}$ at the Ca infrared triplet) after accounting for the 0.7 times anamorphic magnification factor, and a spectral coverage of 6500-9100 $\AA$ (which varies a little from slit to slit depending on their particular placement within the mask field of view). The total integration times were 80 minutes for field KD1 and 115 minutes for field KD2, divided into four and five separate exposures, respectively, for cosmic-ray removal. Typical DEIMOS spectra for different magnitude stars are shown in Figure 12. The typical seeing during the Leo I spectroscopic observations was $0.8^{\prime \prime}-1.0^{\prime \prime}$. All steps of the data reduction to wavelength-calibrated, one-dimensional spectra (including bias subtraction, flat-fielding, sky subtraction, wavelength solutions, and extraction of onedimensional spectra) were done using the spec $2 \mathrm{~d}$ reduction pipeline (M. C. Cooper et al. 2007, in preparation) developed at the University of California at Berkeley for the DEEP2 galaxy redshift survey project.

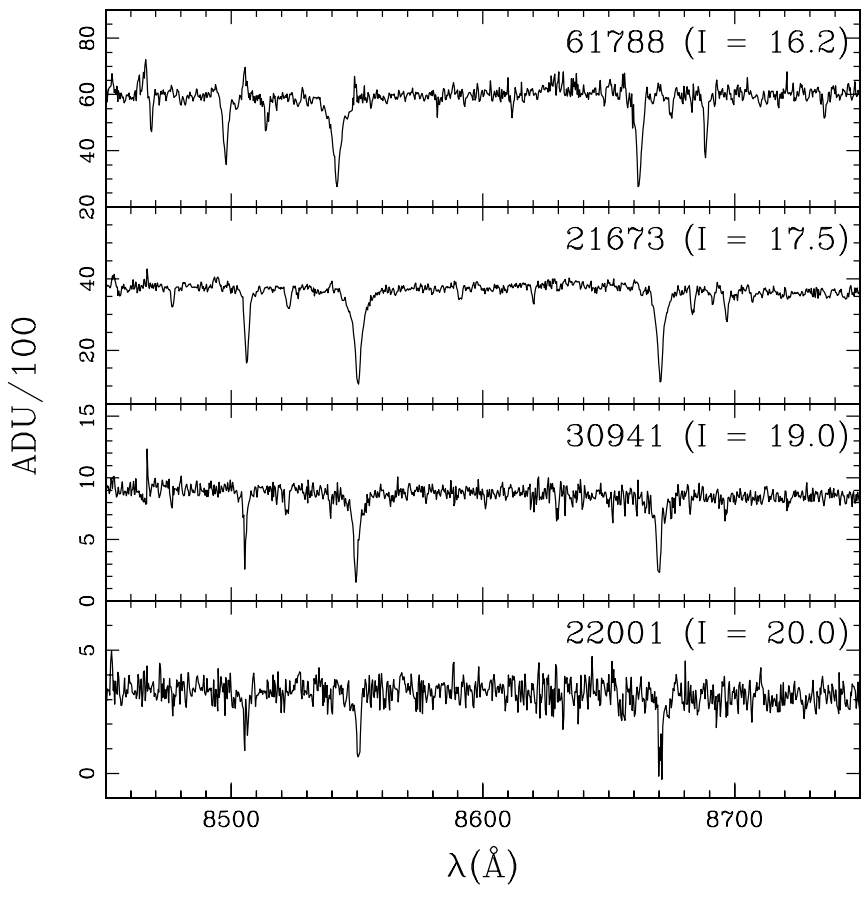

FIG. 12.- Spectra of four stars observed with the Keck DEIMOS. The wavelength region $8450-8750 \AA$ shown highlights the Ca triplet lines. The top spectrum is that of our chosen RV template star (see text), and the bottom three stars are Leo I giants of different brightnesses.

\subsection{Radial Velocity Determination}

Derivation of the RV measurements uses a sequence of steps, each devised to address some aspect of noise suppression or bias compensation. Combined, the resulting scatter in the achieved RV measurements can be shown to be less than 1/40 of the FWHM of a resolution element for the best exposed Leo I stars.

The first phase of the RV reduction follows the masked, Fourierfiltered cross-correlation process outlined in Majewski et al. (2004) and used in a number of our RV studies over more than a decade (e.g., Kunkel et al. 1997). Briefly, each star is first Fourier filtered to attenuate frequency components lower than those given by the typical absorption line and higher than permitted by the intrinsic line resolution (as might be introduced by cosmic rays, for example). Masking multiplies a filtered spectrum in wavelength space with zero to suppress, or with unity to admit portions of a spectrum. Mask wavelengths are selected according to several line lists that each serve specific functions. For example, an $\mathrm{OH}$ night-sky emission mask multiplies all candidate data spectra prior to the correlation process to suppress possible residual noise due to imperfect night-sky subtraction. Another mask similarly multiplies filtered spectra prior to correlation to suppress telluric water and $\mathrm{O}_{2}$ bands when their strength at the instrumental resolution exceeds a few percent. Finally, a third line list is used to mask the cross-correlation "master template," which is set to zero everywhere except at low-ionization or low-excitation metallic or Balmer features that, at the instrumental resolution and at mean target star signal levels, contribute a useful equivalent width component for a metallicity representative of the anticipated population (in this case, Leo I). The suitability of features selected for inclusion in this last mask line list is decided from visual inspection of the Arcturus Atlas (Hinkle et al. 2000), which permits identification of satisfactory features as well as untrustworthy blends (usually from line pairs of dissimilar elements) for exclusion, and with reference to representative target 
star spectra to determine in a practical sense the visibility of the selected features.

In the specific case of our Leo I reductions, our master crosscorrelation template was based on the star 61788 in the KD1 mask, which was very well exposed $\left(\sim 10,000 \mathrm{ADU}_{\text {pixel }}^{-1}\right.$, roughly 20 times stronger than the weaker Leo I spectra; see Fig. 12). This source was used as an RV template because no exposure of a more standard RV reference star was obtained, since the Keck observations of Leo I were obtained only as a backup program for the DEEP project. The benefit of using this particular star as a cross-correlation template is its close match in spectral type and metallicity with the Leo I spectra. The disadvantage is that the true RV of this local reference is unknown, so the final RVs derived in the initial cross-correlation phase are subject to zero-point uncertainties, although their random errors are low. Since the key dynamical descriptors of the Leo I population of relevance to our later analysis are based on relative distributions of RVs, any systemic RV offset present in the data does not explicitly affect our interpretations. The correction of systematic velocity offsets to a known velocity system can be achieved by accounting for offsets against telluric line references, as described below. In addition, we describe how we ultimately adjust to the zero point of the M98 RV system in $\S 5.3$, a necessary additional step if we wish to combine the two data sets.

In preparing star 61788 to serve as a cross-correlation template, the masking procedure left predominantly the lines of the $\mathrm{Ca}$ infrared triplet, as well as the stronger features of $\mathrm{Mg}, \mathrm{Fe}, \mathrm{Ti}, \mathrm{Ni}, \mathrm{Si}$, $\mathrm{Na}$, and $\mathrm{H} \alpha$. These lines visible in the atmosphere of the star 61788 were preserved with the nonzero mask "slats" represented by a Gaussian of unit amplitude and a width roughly 1.2 times the instrument profile. A total of 85 lines were used in crosscorrelations using this masked template, with each slat having an FWHM of $3.0 \AA$ for a total used spectral range of $255 \AA$ over the full spectral range. Experiments using slats of 2.5 and $4.2 \AA$ yielded no significant variations in the quality of the crosscorrelation results. As described above, before cross-correlation the other Leo I stars have been similarly Fourier filtered to the 61788 spectrum. To describe the quality of each cross-correlation, we have adopted the RV quality index $Q$, which is a descriptor of the shape of the central cross-correlation peak with respect to sideband peaks. ${ }^{18}$ We note that although the meaning of the RV quality values is identical to that given in Majewski et al. (2004), the actual values of the cross-correlation peak (CCP) levels are on a relative scale that is only meaningful within the context of the present instrument setup. In general, only those RVs with $Q \geq 4$ can be trusted as reliable, and we discard any star that does not meet this criterion in subsequent analysis.

A primary limitation on the precision of RVs as just obtained is the nonuniform manner in which a spectrometer slit is illuminated, even when the star is perfectly centered in the slit. The presumption that a spectrometer slit is uniformly illuminated is true only when the illuminating source is uniformly spread over the portion of sky that the telescope is sampling. Even in poor seeing a stellar point source, through spread over an angle with a roughly Gaussian profile on the sky, tends to illuminate the slit aperture (along dispersion) differently compared to the illumination from a lamp used to impose pieces of monochromatic light for wavelength calibration. To the extent that this difference appears perpendicular to the slit aperture, there is a displacement in the centroid of (monochromatic) stellar light along the dispersion compared with that of light from a uniformly illuminated

\footnotetext{
18 See Majewski et al. (2004) and Kunkel et al. (1997) for descriptions on how $Q$-values are assigned.
}

source (such as the sky or a calibrating lamp). The effects are obviously greatly amplified in the case that the star is not centered in the slit, whether due to random errors in the astrometry, imperfect tooling of the mask slits, and/or mask misalignment at the time of observation. The dispersion displacement varies with the circumstances of an exposure and can be determined only from signatures carried by the stellar signal itself. Fortunately, the signature of this displacement is also imposed on absorption features originating from molecules of telluric $\mathrm{O}_{2}$ and $\mathrm{H}_{2} \mathrm{O}$ in the light path, and whose profiles and positions accurately track the mean integrated slit function of each particular star as passed through its slit. Thus, the mean wavelength offsets in telluric features can be used to derive slit displacements on a star-by-star basis. To derive the telluric offsets, we design a new cross-correlation mask in which all spectral features are blocked except the regions with the strongest telluric absorption features. The wavelength ranges on which the telluric offset determination is based are 6866$6912 \AA$ (the Fraunhofer B band), 7167-7320,, $7593-7690 \AA$ (the Fraunhofer A band), 8110-8320 , and 8925-9120 A, but all telluric features weaker than $20 \%$ were not used. A "master" template of telluric features was made from a fairly strong Keck spectrum of a star with weak stellar features. Unlike stellar features, the telluric features will look identical in all stars (modulo the $\mathrm{S} / \mathrm{N}$ of their spectra), and so the dominant peak profile in cross-correlations with our masked telluric spectrum should appear the same. This was confirmed visually, and the lowest $Q$-values we would assign to any of these cross-correlation peaks would be a " 6 ;" even stellar spectra with bad $Q$ from the cross-correlation of stellar features still provide excellent telluric correlation profiles. We note that application of the offsets derived from crosscorrelation of telluric features also corrects the original RVs for errors in the pixel-to-wavelength calibration and, in addition, places the RVs to an absolute reference.

The heliocentric RVs corrected for these offsets (with the additional zero-point correction to the Leo I systemic velocity as derived in $\S 5.3$ ) are given in column (8) of Table 4, along with their right ascension and declination coordinates, $M_{0}$ magnitudes, and $\left(M-T_{2}\right)_{0}$ and $(M-\text { DDO51) })_{0}$ colors in columns (3) $-(7)$. We also list galactocentric standard of rest (GSR) RVs, ${ }^{19}$ telluric offsets, cross-correlation peaks (CCP), and quality index $(Q)$ in columns (9)-(12) of Table 4.

For those stars observed in $1^{\prime \prime}$ wide slits, ${ }^{20}$ the mean telluric offset is $-11.5 \mathrm{~km} \mathrm{~s}^{-1}$ with a dispersion of $7.7 \mathrm{~km} \mathrm{~s}^{-1}$ for those stars in mask KD1 and $-2.8 \mathrm{~km} \mathrm{~s}^{-1}$ with a dispersion of $3.5 \mathrm{~km} \mathrm{~s}^{-1}$ for those stars in mask KD2. That both mean offsets are nonzero is a reflection of systematics in the original template derived from star 61788; that the mean offsets are different for each mask reflects the differences in positioning of each mask relative to their respective star fields. The 7.7 and $3.5 \mathrm{~km} \mathrm{~s}^{-1}$ dispersions are, respectively, equivalent to $\sim 0.2^{\prime \prime}$ and $\sim 0.1^{\prime \prime}$ variations in the slit centering and reflect the quality of the original astrometry (see $\S 2$ ), the slit manufacture, and the rotational alignment of the slit mask on the sky. Figure 13, which shows the telluric offset correction for both masks as a function of position along the mask (approximately declination for mask KD1 and right ascension for mask KD2), demonstrates, however, that the initial astrometric reduction contributes significantly to the star-by-star slit miscentering, given the

\footnotetext{
19 We convert to galactocentric standard of rest by assuming a local standard of rest velocity of $220 \mathrm{~km} \mathrm{~s}^{-1}$ and a solar peculiar velocity of $(u, v, w)=$ $(-9,12,7) \mathrm{km} \mathrm{s}^{-1}$

${ }^{20}$ Seven stars listed in Table 4 were actually alignment stars observed in $4^{\prime \prime}$ wide slits. However, with the telluric offset corrections we can actually derive good RVs for these spectra with larger equivalent slit functions. The larger telluric offset corrections needed for these stars are evident in the tabulated values.
} 
TABLE 4

Observed Radial Velocities of Stars

\begin{tabular}{|c|c|c|c|c|c|c|c|c|c|c|c|}
\hline $\begin{array}{c}\text { Slit Number } \\
\text { (1) }\end{array}$ & $\begin{array}{l}\text { ID } \\
(2)\end{array}$ & $\begin{array}{c}\text { R.A. } \\
\text { (J2000.0) } \\
(3)\end{array}$ & $\begin{array}{c}\text { Decl. } \\
(\mathrm{J} 2000.0) \\
(4)\end{array}$ & $\begin{array}{l}M_{0} \\
(5)\end{array}$ & $\begin{array}{c}\left(M-T_{2}\right)_{0} \\
(6)\end{array}$ & $\begin{array}{c}(M-\mathrm{DDO} 51)_{0} \\
(7)\end{array}$ & $\begin{array}{c}v_{\text {hel }^{\mathrm{a}}} \\
\left(\mathrm{km} \mathrm{s}^{-1}\right) \\
(8)\end{array}$ & $\begin{array}{c}v_{\mathrm{GSR}}{ }^{\mathrm{b}} \\
\left(\mathrm{km} \mathrm{s}^{-1}\right) \\
(9)\end{array}$ & $\begin{array}{l}\text { Tell. Off. } \\
\left(\mathrm{km} \mathrm{s}^{-1}\right) \\
(10)\end{array}$ & $\begin{array}{c}\mathrm{CCP}^{\mathrm{d}} \\
(11)\end{array}$ & $\begin{array}{c}Q^{\mathrm{e}} \\
(12)\end{array}$ \\
\hline KD1-00 ........................... & $61782^{\mathrm{f}}$ & 100733.33 & +122620.1 & 18.69 & 1.88 & -0.23 & 0.5 & -106.6 & -41.4 & 0.59 & 5 \\
\hline KD1-01 ............................ & $61736^{\mathrm{f}}$ & 100740.39 & +122627.7 & 18.17 & 1.65 & -0.20 & 39.3 & -67.8 & -35.9 & 0.40 & 6 \\
\hline KD1-02 …........................ & $72520^{\mathrm{f}}$ & 100730.84 & +121341.8 & 17.99 & 0.79 & 0.03 & 158.6 & 50.7 & -17.8 & 0.39 & 4 \\
\hline 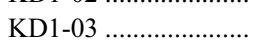 & $72418^{\mathrm{f}}$ & 100744.50 & +121236.8 & 16.44 & 0.81 & 0.04 & -13.1 & -121.0 & -18.9 & 0.50 & 5 \\
\hline 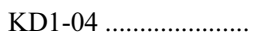 & 61774 & 100734.30 & +122509.4 & 18.93 & 2.90 & -0.04 & 62.5 & -44.7 & -16.0 & 0.46 & 5 \\
\hline KD1-05 ….................. & 61788 & 100731.61 & +122330.4 & 17.56 & 1.46 & -0.17 & 26.2 & -81.1 & -23.5 & 0.59 & 5 \\
\hline KD1-06 ..................... & 61770 & 100734.86 & +122515.0 & 18.77 & 2.34 & -0.17 & 12.4 & -94.8 & -22.8 & 0.57 & 6 \\
\hline 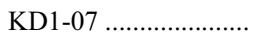 & 61785 & 100732.43 & +122450.0 & 19.84 & 3.14 & -0.04 & 10.9 & -96.3 & -30.7 & 0.34 & 4 \\
\hline 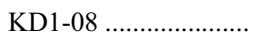 & 72466 & 100738.44 & +121333.4 & 20.00 & 2.84 & -0.02 & 24.0 & -83.9 & -14.5 & 0.53 & 6 \\
\hline 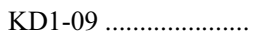 & 61692 & 100745.33 & +122347.6 & 20.56 & 2.96 & -0.08 & 4.0 & -103.3 & -23.9 & 0.50 & 6 \\
\hline KD1-10 .................... & 72449 & 100740.94 & +121303.6 & 20.11 & 1.82 & 0.02 & 288.3 & 180.4 & -8.0 & 0.91 & 6 \\
\hline KD1-11_..................... & 72472 & 100737.98 & +121744.3 & 20.19 & 1.65 & 0.01 & 293.5 & 185.9 & -14.0 & 0.87 & 6 \\
\hline KD1-12 … & 72456 & 100739.52 & +121654.6 & 20.24 & 1.58 & 0.04 & 303.1 & 195.4 & -9.5 & 0.64 & 7 \\
\hline KD1-13 .................... & 61733 & 100740.43 & +122023.4 & 20.19 & 1.50 & 0.03 & 288.1 & 180.6 & -11.2 & 0.80 & 6 \\
\hline 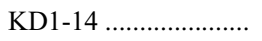 & 72526 & 100730.06 & +121621.6 & 20.41 & 1.48 & 0.00 & 290.1 & 182.4 & -10.8 & 0.82 & 6 \\
\hline KD1-15 ….................. & 72493 & 100734.86 & +121243.9 & 20.57 & 1.51 & 0.00 & 291.8 & 183.9 & -10.4 & 0.75 & 6 \\
\hline KD1-16 ...................... & 72463 & 100738.60 & +12 1128.7 & 20.55 & 1.47 & 0.04 & 278.9 & 170.9 & -9.0 & 0.74 & 6 \\
\hline KD1-17 .................... & 61790 & 100730.46 & +122132.6 & 20.65 & 1.53 & 0.02 & 302.0 & 194.6 & -12.9 & 0.53 & 6 \\
\hline KD1-18 ..................... & 61757 & 100737.01 & +122242.9 & 20.77 & 1.48 & 0.01 & 289.1 & 181.8 & -13.4 & 0.65 & 7 \\
\hline 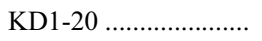 & 72407 & 100745.47 & +121532.7 & 21.31 & 1.30 & 0.02 & 274.8 & 167.1 & -5.7 & 0.49 & 7 \\
\hline KD1-21 ............................ & 72432 & 100743.00 & +121627.9 & 21.37 & 1.33 & 0.05 & 293.4 & 185.7 & -3.7 & 0.49 & 6 \\
\hline 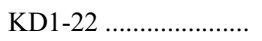 & 61783 & 100732.88 & +122101.9 & 21.42 & 1.35 & 0.01 & 294.6 & 187.1 & -14.6 & 0.52 & 6 \\
\hline 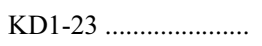 & 61755 & 100737.37 & +122227.3 & 21.41 & 1.32 & 0.07 & 313.9 & 206.5 & -16.2 & 0.36 & 6 \\
\hline KD1-24 .................... & 61753 & 100737.44 & +121918.2 & 21.49 & 1.59 & 0.22 & 283.3 & 175.8 & +3.8 & 0.35 & 6 \\
\hline KD1-25 ..................... & 61746 & 100738.47 & +121927.3 & 21.93 & 1.26 & 0.11 & 288.1 & 180.6 & -11.3 & 0.28 & 4 \\
\hline 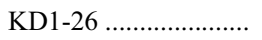 & 72464 & 100738.53 & +12 1311.0 & 21.56 & 1.21 & 0.06 & 290.9 & 183.0 & -9.0 & 0.34 & 6 \\
\hline KD1-27 ....................... & 72525 & 100730.36 & +121144.5 & 21.62 & 1.27 & 0.06 & 314.8 & 206.8 & -8.2 & 0.25 & 5 \\
\hline KD1-28 …................. & 61769 & 100734.97 & +122423.2 & 21.40 & 3.42 & 0.07 & 53.2 & -54.1 & -14.5 & 0.42 & 6 \\
\hline KD1-29 ...................... & 72505 & 100733.39 & +121100.4 & 18.90 & 0.85 & 0.03 & 178.3 & 70.3 & -9.7 & 0.42 & 5 \\
\hline KD1-30 ..................... & 61743 & 100739.55 & +122639.4 & 18.98 & 0.81 & 0.03 & 129.7 & 22.6 & -14.8 & 0.28 & 4 \\
\hline KD1-32 … & 72490 & 100735.03 & +121548.2 & 19.88 & 1.45 & -0.14 & 52.7 & -55.0 & -6.3 & 0.57 & 6 \\
\hline KD1-33 ...................... & 61707 & 100743.58 & +122121.2 & 19.41 & 0.91 & 0.06 & 219.1 & 111.7 & -14.7 & 0.18 & 4 \\
\hline KD1-34 …................... & 61694 & 100744.99 & +121952.8 & 21.70 & 3.14 & 0.03 & 24.2 & -83.3 & -9.1 & 0.47 & 6 \\
\hline KD1-36 ...................... & 61766 & 100735.12 & +122205.2 & 20.88 & 2.10 & -0.22 & 68.6 & -38.8 & -5.9 & 0.57 & 6 \\
\hline KD1-37 .................... & 61772 & 100734.39 & +121857.0 & 20.78 & 1.76 & -0.24 & 74.9 & -32.7 & +4.1 & 0.48 & 6 \\
\hline KD1-38 ..................... & 61775 & 100734.18 & +122252.2 & 20.72 & 1.29 & -0.06 & -52.6 & -159.9 & -21.0 & 0.48 & 4 \\
\hline KD1-39 ...................... & 61724 & 100741.49 & +122316.0 & 21.44 & 1.85 & -0.22 & 24.9 & -82.4 & -19.2 & 0.44 & 5 \\
\hline KD1-40 ..................... & 72531 & 100729.23 & +121159.5 & 20.38 & 0.76 & 0.05 & 318.1 & 210.1 & -10.3 & 0.18 & 4 \\
\hline KD1-42 …................... & 72439 & 100742.05 & +12 1120.8 & 21.21 & 1.27 & 0.00 & 307.5 & 199.5 & -13.7 & 0.35 & 5 \\
\hline KD1-45 ...................... & 72508 & 100732.88 & +121558.1 & 21.43 & 1.22 & -0.01 & 282.7 & 175.0 & -1.1 & 0.59 & 7 \\
\hline KD1-46 ...................... & 72455 & 100739.82 & +121755.1 & 21.53 & 1.31 & -0.04 & 316.1 & 208.5 & +6.7 & 0.31 & 4 \\
\hline KD1-50 .................... & 61792 & 100730.07 & +122220.6 & 21.88 & 1.13 & 0.03 & 305.3 & 197.9 & -11.5 & 0.34 & 6 \\
\hline KD2-03 ....................... & 21673 & 100836.93 & +122011.5 & 19.47 & 1.99 & -0.02 & 299.0 & 191.7 & -2.8 & 1.08 & 7 \\
\hline KD2-04 ........................ & 20868 & 100844.71 & +122020.9 & 19.79 & 1.72 & 0.04 & 292.2 & 185.0 & -1.9 & 0.93 & 6 \\
\hline KD2-05 ….................. & 23429 & 100825.15 & +122007.5 & 19.85 & 1.78 & -0.02 & 294.0 & 186.7 & -4.9 & 1.00 & 6 \\
\hline KD2-06 ..................... & 31007 & 100840.95 & +121806.7 & 19.94 & 1.83 & 0.03 & 293.3 & 185.9 & -1.9 & 0.88 & 7 \\
\hline KD2-07 ..................... & 20898 & 100844.30 & +121956.3 & 19.89 & 1.78 & 0.03 & 285.3 & 178.0 & -4.1 & 0.93 & 7 \\
\hline 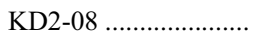 & 20945 & 100843.74 & +12 1957.9 & 19.84 & 1.70 & 0.03 & 296.9 & 189.6 & -3.8 & 0.98 & 6 \\
\hline 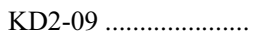 & 23059 & 100827.55 & +122020.9 & 19.81 & 1.65 & -0.02 & 276.9 & 169.6 & -1.5 & 0.80 & 7 \\
\hline KD2-11..................... & 20198 & 100907.46 & +121838.7 & 19.99 & 1.77 & 0.01 & 291.2 & 183.9 & -5.0 & 0.88 & 6 \\
\hline 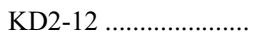 & 20231 & 100905.15 & +122014.4 & 20.05 & 1.73 & 0.01 & 288.8 & 181.6 & -6.9 & 0.94 & 6 \\
\hline KD2-13 ................... & 20345 & 100858.71 & +121836.4 & 20.07 & 1.70 & 0.00 & 284.5 & 177.2 & -4.8 & 0.88 & 7 \\
\hline KD2-14 ...................... & 20982 & 100843.32 & +122123.6 & 20.10 & 1.72 & 0.05 & 286.0 & 178.8 & -5.3 & 0.80 & 6 \\
\hline KD2-15 ..................... & 22396 & 100831.81 & +121956.0 & 20.00 & 1.58 & -0.02 & 287.5 & 180.2 & +0.6 & 0.70 & 6 \\
\hline KD2-16 .................... & 20129 & 100914.89 & +121835.0 & 20.10 & 1.65 & 0.01 & 275.5 & 168.3 & -8.3 & 0.74 & 7 \\
\hline KD2-17 ….................... & 20248 & 100904.17 & +122046.0 & 20.13 & 1.68 & 0.03 & 292.0 & 184.8 & -11.7 & 0.93 & 6 \\
\hline KD2-18 ..................... & 23131 & 100827.15 & +122006.3 & 20.09 & 1.63 & 0.05 & 280.1 & 172.8 & -5.4 & 0.86 & 6 \\
\hline KD2-19 ...................... & 31135 & 100839.39 & +12 1723.8 & 20.13 & 1.67 & 0.09 & 286.7 & 179.3 & +1.2 & 0.81 & 6 \\
\hline KD2-20 .................... & 70295 & 100822.67 & +121721.3 & 20.21 & 1.75 & 0.00 & 287.2 & 179.7 & -6.4 & 0.42 & 5 \\
\hline 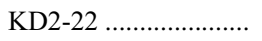 & 21617 & 100837.29 & +122012.0 & 20.20 & 1.68 & 0.01 & 291.9 & 184.6 & -1.5 & 0.90 & 6 \\
\hline 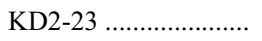 & 60019 & 100824.25 & +121853.5 & 20.20 & 1.65 & 0.03 & 284.0 & 176.6 & +4.2 & 0.84 & 6 \\
\hline KD2-24 ........................ & 30123 & 100913.77 & +121723.0 & 20.28 & 1.71 & 0.04 & 296.9 & 189.6 & -6.1 & 0.75 & 6 \\
\hline KD2-25 ……............... & 30522 & 100849.87 & +12 1801.2 & 20.21 & 1.61 & 0.04 & 291.4 & 184.0 & +0.9 & 0.79 & 6 \\
\hline KD2-26 .................... & 60310 & 100821.89 & +12 1933.4 & 20.25 & 1.63 & 0.02 & 280.4 & 173.0 & +2.4 & 0.79 & 6 \\
\hline KD2-27 ..................... & 30102 & 100915.88 & +121718.5 & 20.39 & 1.74 & 0.03 & 284.1 & 176.8 & -3.8 & 0.80 & 6 \\
\hline KD2-28 . & 21961 & 100834.69 & +12 1911.4 & 20.27 & 1.56 & 0.03 & 283.6 & 176.3 & -1.1 & 0.82 & 6 \\
\hline
\end{tabular}


TABLE 4-Continued

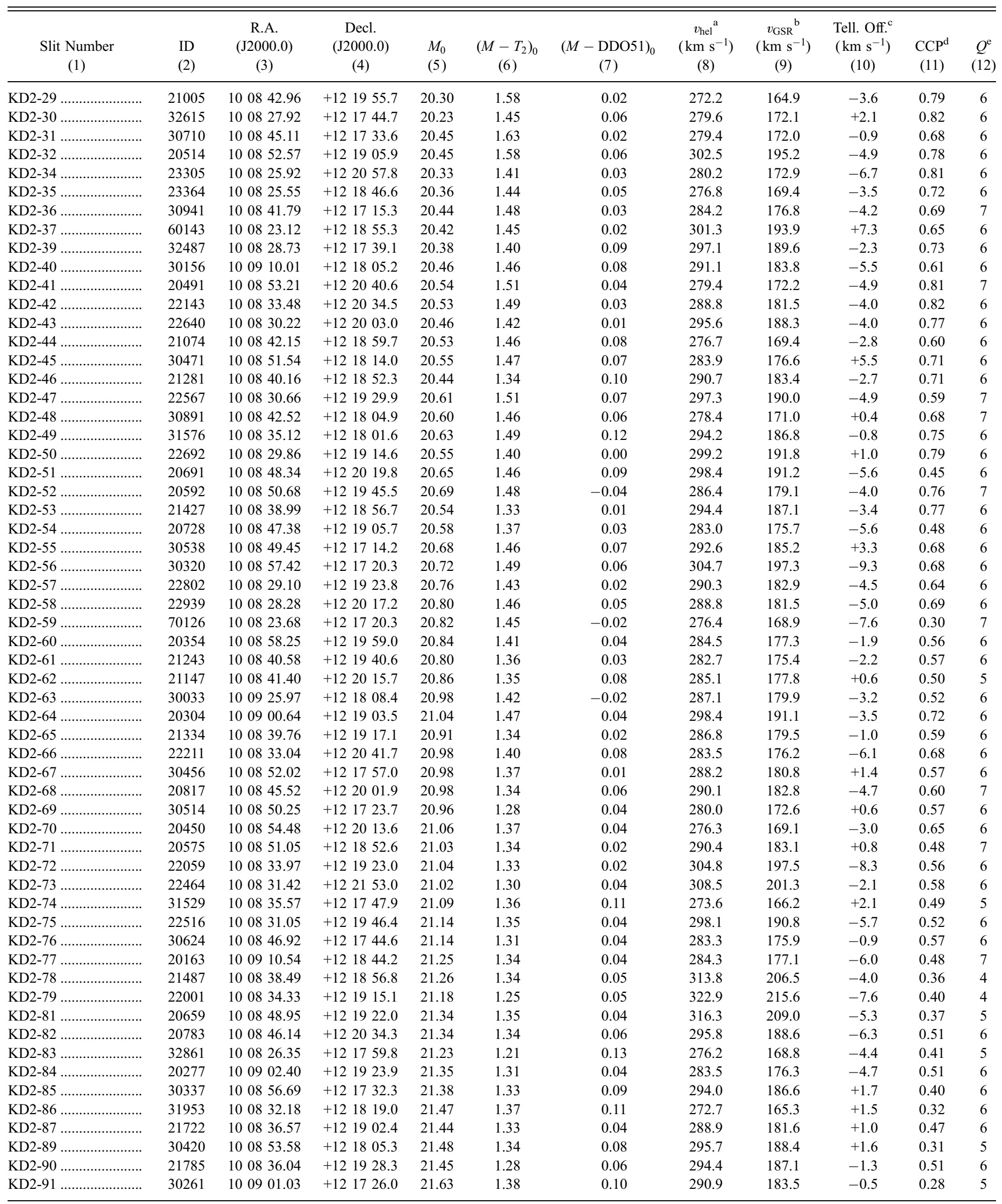

Notes.-Units of right ascension are hours, minutes, and seconds, and units of declination are degrees, arcminutes, and arcseconds. Table 4 is also available in machinereadable form in the electronic edition of the Astrophysical Journal.

${ }^{a}$ Heliocentric RVs corrected for the telluric offsets as described in text.

${ }^{b}$ RVs in galactocentric standard of rest.

c Telluric offsets; see text.

${ }^{\mathrm{d}}$ Cross-correlation peaks.

e Quality indicator; see text.

f Alignment stars observed with $4^{\prime \prime}$ wide slits. All other stars were observed with $1^{\prime \prime}$ wide slits. 

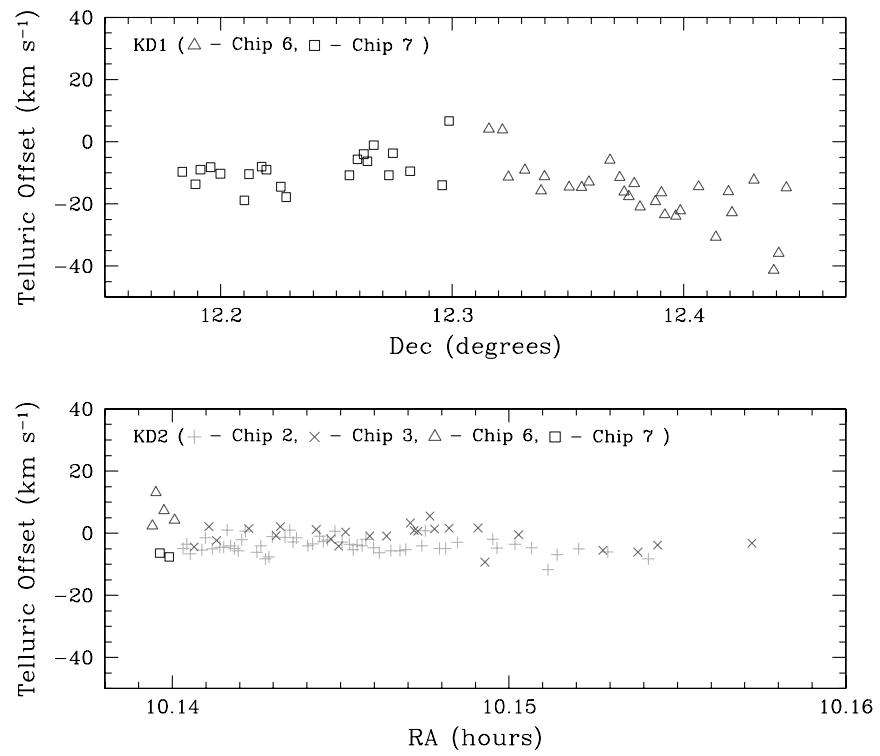

FIG. 13.-Telluric offsets for each stellar RV measurement as a function of the position in the mask for KD1 (top) and KD2 (bottom). The variation in astrometric solution from chip to chip in the original Mosaic camera data is revealed by the trends in telluric offset by chip number. [See the electronic edition of the Journal for a color version of this figure.]

clear correlations of the offset trends with the original CCD frame on which the star was originally photometered (each CCD frame has a unique astrometric solution). The dispersions in the telluric offsets represent the actual precision limits for multislit RVs in the case when the telluric offsets are not accounted for. After applying the telluric offset, the velocity precision is therefore well better than this.

\subsection{Determination of Sampling Errors}

Based on the relative strengths of the cross-correlations against the stellar and telluric templates, the dominant contribution to the $\mathrm{RV}$ errors is in the stellar absorption cross-correlation; the telluric cross-correlation peaks are almost always much stronger than the kinematic correlation peaks because the equivalent widths of telluric absorption features sum to an order of magnitude larger than those intrinsic to the stellar atmosphere. Errors in the stellar absorption cross-correlation are partly a reflection of differences in stellar line strengths between the template and target spectra.

An assessment of random sampling errors may be approached by a variety of techniques; however, the method of Tonry \& Davis (1979) is not one of them. The adopted cross-correlation methodology employed here differs from that of Tonry \& Davis (1979), having evolved over years of experience and motivated by the goal to cover as broad a range of (generally unknown) spectral types among the target stars as possible. The Tonry \& Davis (1979) method relies explicitly on the characteristic that a template and a target spectrum must be of identical shape, or spectral type, so that the resulting cross-correlation ideally becomes an even function with null imaginary terms. Then the imaginary terms that appear for real data may be utilized for the estimation of sampling errors. In contrast, our methodology, by using slat masking, maximizes the tolerance of the cross-correlation to widely variable spectral types, so that $\mathrm{F}$ through $\mathrm{M}$ stars have in the past been measured equally successfully to full precision in the same correlation process with a single template design. But such dissimilarities invalidate the reliance on the even character of the correlation function, and imaginary terms are generally nonzero even when sampling errors are rigorously zero. Conse- quently, alternate methods for estimating errors with our methodology are required.

To obtain a first estimate of the errors in our RVs, we repeated the stellar absorption cross-correlations twice with other RV templates manufactured from other stars in the Leo I masks with much stronger stellar absorption features than star 61788, namely, stars 61736 and 61782 . With the cross-correlations there was little difference in the final results for the best-quality target spectra, and there were only minor changes in $Q$-values using different $\mathrm{RV}$ templates. For $Q=7$ spectra the rms scatter in RVs was $1.2 \mathrm{~km} \mathrm{~s}^{-1}$. This rises to $3.0 \mathrm{~km} \mathrm{~s}^{-1}$ when any one of the cross-correlation peaks dropped to $Q=4$. This analysis suggested an uncertainty scale of $1.2,1.5,2.0$, and $3.0 \mathrm{~km} \mathrm{~s}^{-1}$ for stars with $Q=7,6,5$, and 4 , respectively. However, these uncertainties must be regarded as lower limits, since the use of different templates on the same target spectra does not lead to completely statistically independent measurements.

Because repeat observations of our target stars are not available, an alternative error estimation derives from creating artificial "repeat observations" via the convolution of the raw, unfiltered candidate spectra with randomized Poisson noise. Practically, this is accomplished by using the original spectrum as a probability distribution function, whereby, at any wavelength, the envelope of the original spectrum defines the "expectation" of received source counts with respect to neighboring wavelengths. With each wavelength "bin" set to the resolution FWHM width, the spectrum can be repopulated with Poisson-distributed photon events, dropped in randomly with wavelength destinations weighted according to the expectation probabilities. To get a representative spectrum whose noise characteristics are like those of the raw data (presuming a negligible contribution from read noise), one keeps adding Poisson events until the count of events equals that of the raw target spectrum. On average, each wavelength bin in the new pseudospectrum retains the same amplitude, compared to neighbors, as the original spectrum, with modulation only due to Poisson noise. When a number of pseudospectra are so rebuilt but arbitrarily scaled to a much stronger total photon count than the original spectrum signal strength, their cross-correlations with the template show zero velocity scatter, as one expects for strong signals. When, on the other hand, Poisson-distributed photons are dropped into each slat so that the overall mean exposure level of the pseudospectra is comparable to the observed spectrum, the scatter in the derived velocities is found to be identical to that obtained by reobserving the same star as many times. ${ }^{21}$

The above error estimation method was applied to a variety of Leo I spectra with the generated pseudospectra cross-correlated against the same RV template using the same masks. This procedure packages into one procedure a test of all aspects of the data processing stream, including all instrumental traits, as well as the design of the line lists, template fabrication, etc. If we adopt test spectra near the low-end limit of signal levels, i.e., $\sim 500 \mathrm{ADU}$, we obtain dispersions of $1.55,1.77,2.87$, and $4.91 \mathrm{~km} \mathrm{~s}^{-1}$ for stellar feature cross-correlations in $Q=7,6,5$, and 4 spectra, respectively. Obviously, better precisions are achieved for the large number of spectra with higher signal levels; e.g., for $Q=7$ spectra, dispersions of $0.44,0.50$, and $1.46 \mathrm{~km} \mathrm{~s}^{-1}$ were obtained for signal levels of about 4200, 2500, and 1000 ADU. Independent tests of the telluric cross-correlations at these signal strengths

21 The basis for this claim derives from tests of this methodology on other data sets available to the authors wherein multiple observations of the stars are available. In all cases tested, equivalent RV scatters were found for multiple observations of the same star compared to multiple pseudospectra generated from one observation. 


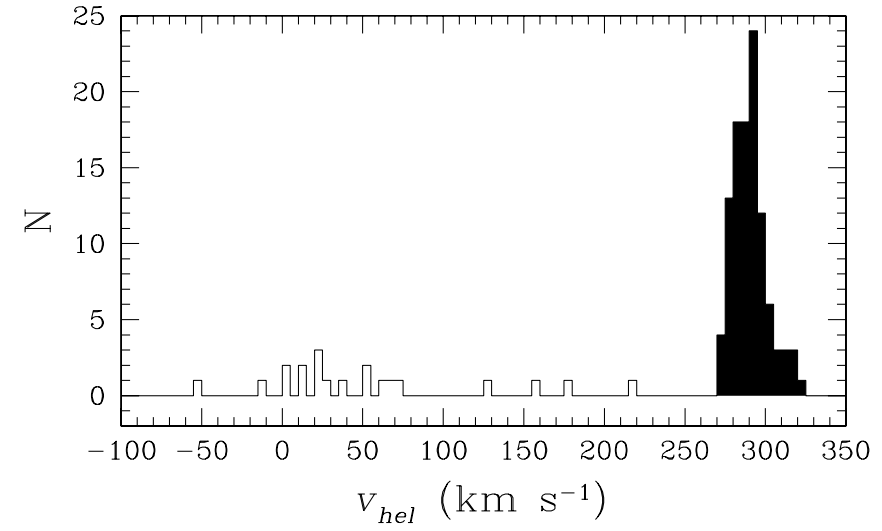

FIG. 14.-Histogram for heliocentric RVs of 125 stars observed with Keck DEIMOS that have $Q \geq 4$. Bin size is $5 \mathrm{~km} \mathrm{~s}^{-1}$.

lead to scatters of about $1.2 \mathrm{~km} \mathrm{~s}^{-1}$ (and significantly better for stronger spectra). Adding these contributions in quadrature, and conservatively adopting the worst-case (i.e., low signal strength) dispersions for each quality, leads to the following estimated uncertainties for stars with $Q=7,6,5$, and 4, respectively: 2.0, $2.1,3.1$, and $5.1 \mathrm{~km} \mathrm{~s}^{-1}$. We adopt these as our velocity uncertainties for subsequent analyses. However, it is worth pointing out that by adopting conservative estimates of our uncertainties at every level, we do risk underestimating derived true velocity dispersions after we remove the contributions from observational uncertainties. $^{22}$

\subsection{Testing the Photometric Selection of Leo I Giants}

The extreme systemic RV of Leo I, $v_{\text {hel }}=287 \mathrm{~km} \mathrm{~s}^{-1}$ (M98), is a great advantage for distinguishing true Leo I members from Galactic stars: very few Galactic stars are expected to have such extreme velocities at this position in the Galaxy $\left[(l, b)=\left(226^{\circ}\right.\right.$, $\left.+49^{\circ}\right)$ ]. Figure 14 shows a histogram of the observed RVs of stars listed in Table 4; stars photometrically selected to be Leo I giant candidates are shaded darkly. Leo I giants are easily identified by the clump at $v_{\text {hel }} /\left(\mathrm{km} \mathrm{s}^{-1}\right)>250$. In KD1 21 out of 42 stars are identified as Leo I members, and in KD2 all 83 stars are Leo I members, based on their RVs. Stars we targeted simply as "mask fillers" tend to have RVs clustering near $0 \mathrm{~km} \mathrm{~s}^{-1}$ as expected for MW disk stars.

In Figure 15 we plot the stars observed with the Keck DEIMOS over the color-color and color-magnitude diagrams with the selection regions shown. In both the color-magnitude and colorcolor diagrams, the stars with measured velocity $v_{\text {hel }}>250 \mathrm{~km} \mathrm{~s}^{-1}$ are generally located within our primary giant selection region, whereas the stars with lower velocities are not; in particular, in the two-color diagram the lower velocity stars all fall along the elbow-shaped, dwarf star locus, suggesting that these stars are all relatively nearby MW dwarfs. Even more gratifying is that for those 96 stars selected to be Leo I giants using both the colorcolor and color-magnitude diagrams and for which we obtained RVs, all of them are Leo I members by the RV criterion; this suggests that the reliability of our selection is very good, and we emphasize that a significant portion of our sample includes stars selected in the very low density outer parts of the Leo I system

\footnotetext{
22 For example, adopting the lower limits to the velocity uncertainties derived from our first estimate above (i.e., $1.2,1.5,2.0$, and $3.0 \mathrm{~km} \mathrm{~s}^{-1}$ for $Q=7-4$ ) raises the central Leo I velocity dispersion (see $\S 5$ ) by about $5 \%$ (from 8.2 to $8.6 \mathrm{~km} \mathrm{~s}^{-1}$ ) and the "cold point" velocity dispersion near the King limiting radius (see $\S 5$ ) by the same fraction (from 3.9 to $4.1 \mathrm{~km} \mathrm{~s}^{-1}$ ).
}

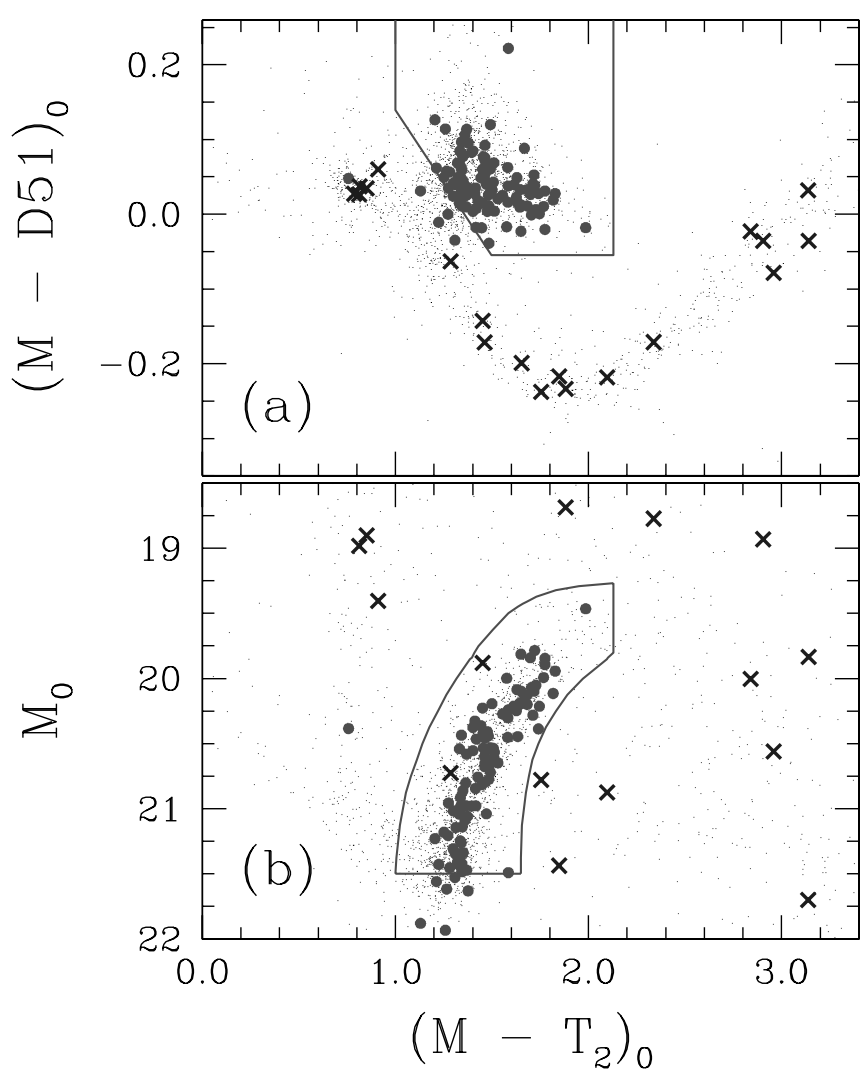

FIG. 15.-Positions of the stars observed with the Keck DEIMOS: $(a)$ in the color-color diagram, and $(b)$ in the CMD. The selection boxes for the Leo I giant candidates (from Figs. 4 and 5) are shown in both plots. The filled circles are for stars that have been confirmed to be members of Leo I based on their heliocentric RVs, while the crosses are for stars confirmed not to be members. [See the electronic edition of the Journal for a color version of this figure.]

(see Fig. 16 below). We also observe that our Leo I giant candidate selection criteria are on the conservative side because nine additional stars that we did not pick as giant candidates are also Leo I RV members. In most cases a slight expansion of our selection criteria would have picked up all but one ${ }^{23}$ of these other Leo I stars, but this expansion would have brought in false positives. In particular, it may be seen that our catalog can be trusted to even fainter magnitudes than the conservative $M_{0}<$ 21.5 limit we adopted for the analyses presented in our photometric studies. It may also be seen that use of the $M-$ DDO5 1 color eliminates contaminant dwarf stars that fall within the colormagnitude locus of the Leo I RGB and thus improves the efficiency of our target selection. This reliability analysis lends credibility to the structural features we have mapped using Leo I giant candidates selected in this way.

Figure 16 shows the sky distribution of the stars in Figure 15. Out of the total of 105 Leo I giants observed with Keck DEIMOS, 90 stars lie within the King limiting radius and 48 stars lie within the core radius, leaving 15 confirmed Leo I members beyond the limiting radius, i.e., in the domain of the break population we identified in our study of the Leo I light profile. The new Keck spectroscopy confirms that our selection of Leo I giant candidates is both effective and reliable and so lends confidence that the population of stars falling outside the King limiting radius (which may be the nominal tidal radius of the system; see $\S 6.1$ ) and

\footnotetext{
23 This one Leo I RV member well outside our color-magnitude selection box at $\left(M-T_{2}\right)_{0}<1.0$ (see Fig. 15) is likely to be an anomalous Cepheid.
} 


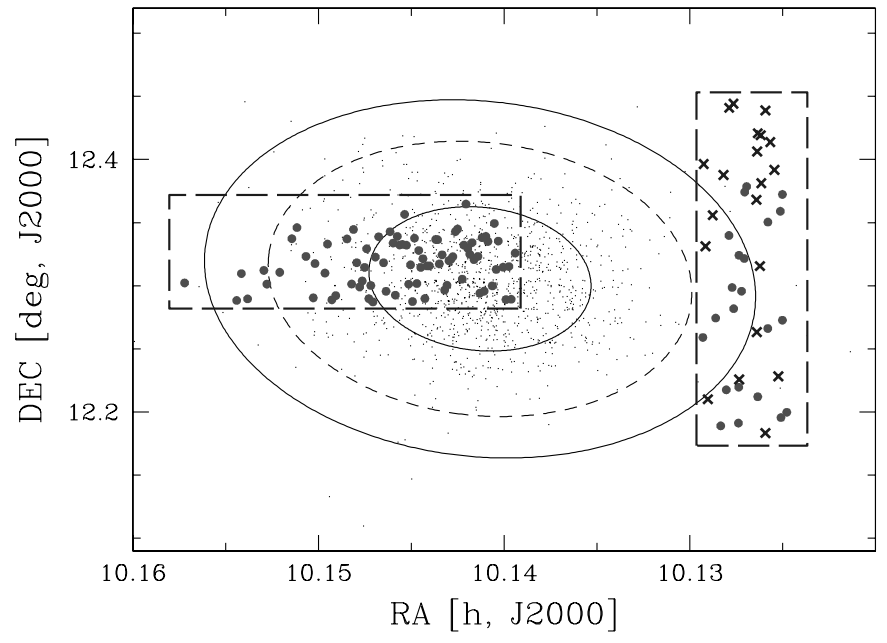

FIG. 16.- Stars observed with the Keck DEIMOS plotted over the sky distribution of Leo I giant candidates. The approximate field of view for the Keck DEIMOS fields is shown with long-dashed lines. The inner ellipse shown in solid line represents the King core radius, and the outer two ellipses are the same as those in Fig. 8. The symbols are the same as those in Fig. 15. [See the electronic edition of the Journal for a color version of this figure.]

constituting our detected break population (e.g., Fig. 9) are actually stars associated with Leo I.

\subsection{Velocity Dispersion}

The variation of velocity dispersion $\left(\sigma_{v}\right)$ with projected radius of dSphs is important for testing dynamical models of dSphs (e.g., Kroupa 1997; Łokas et al. 2005; Strigari et al. 2006b; R. R. Muñoz et al. 2007, in preparation). For a bound stellar system where mass follows light $\sigma_{v}$ is expected to decrease with radius and approach zero at the tidal radius. On the other hand, dSph models with extended DM halos predict a dispersion that falls off more slowly than mass-follows-light models (e.g., Kleyna et al. 2001; Wilkinson et al. 2002). Finally, tidal disruption models predict flat/rising $\sigma_{v}$ profiles (e.g., Kroupa 1997; R. R. Muñoz et al. 2007, in preparation) into the domain dominated by stars that have become unbound during/after tidal interactions with the host galaxy.

With our Keck DEIMOS data, we can explore the radial variation of $\sigma_{v}$ out to and beyond the King limiting radius. Before doing so, we adjust our RVs to the zero point of the M98 RV system so that both data sets may be considered together for improved statistics. The weighted mean $\sigma_{v}$ of the 33 stars observed by M98 is $8.6 \pm 1.2 \mathrm{~km} \mathrm{~s}^{-1}$. These stars lie in the semimajor axis range $r_{\text {maj }} / r_{\text {lim }}=0.055-0.367$ according to our newly derived structural parameters. The $\sigma_{v}$ for 50 stars we observed in the same $r_{\text {maj }}$ range is $8.2 \pm 0.9 \mathrm{~km} \mathrm{~s}^{-1}$, which is consistent with M98's velocity dispersion within the error. ${ }^{24}$ We take as the offset to our $\mathrm{RV}$ system the $+12.4 \mathrm{~km} \mathrm{~s}^{-1}$ difference between the error-weighted mean of our and the M98 samples and apply this offset to the entire Keck DEIMOS data set (although it is not clear, in fact, which of the two surveys is closer to the true absolute RV system). In Figure $17 a$ we plot the heliocentric RVs versus the projected major-axis radial distances for both Keck DEIMOS and M98 samples. For comparison, we have plotted each sample with different symbols (and colors). The remarkable similarity of the RV distributions within $5^{\prime}$ from the center of Leo I assures us that both samples are on the same system. We also show the mean RVs along

\footnotetext{
${ }^{24}$ All velocity dispersion calculations throughout this paper follow the method of Armandroff \& Da Costa (1986).
}

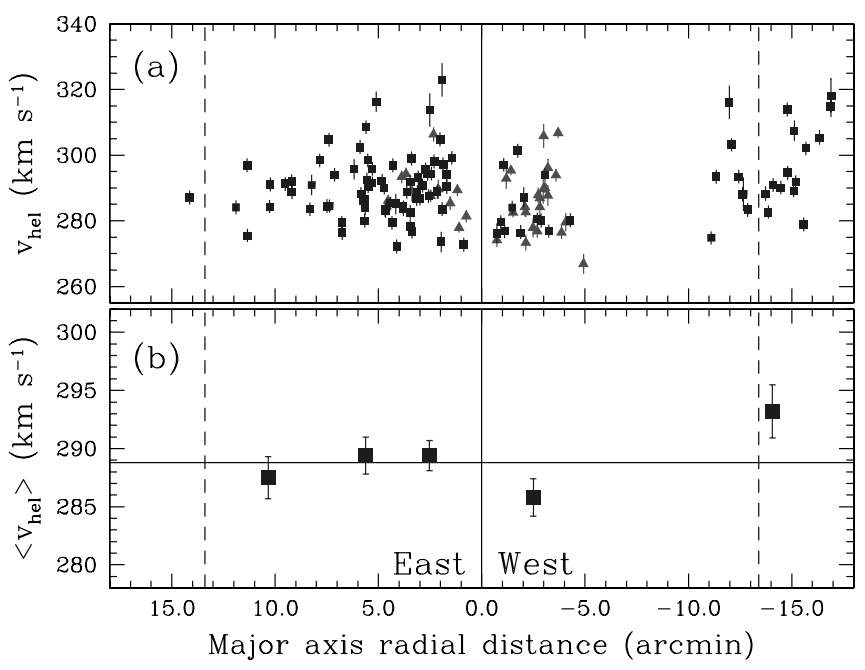

FIG. 17.-Distributions of (a) individual heliocentric RVs and (b) mean RVs along the projected major-axis radial distances. Filled squares in $(a)$ represent stars observed with the Keck DEIMOS, while filled triangles represent those from M98. [See the electronic edition of the Journal for a color version of this figure.]

projected radius in Figure $17 \mathrm{~b}$. The mean RV trend shows that there is a hint of rotation in the inner $<5^{\prime}$, but with low statistical significance. Spectroscopic observations for stars to the west in the range $r_{\text {maj }}=5^{\prime}-10^{\prime}$ will help reveal whether rotation is significant for Leo I. We use the combined sample of M98 and Keck spectroscopy in subsequent analyses.

In Figure 18 we present $v_{\text {hel }}$ and $\sigma_{v}$ as a function of radial distance from the Leo I center, calculated for elliptical (Fig. 18a) and circular radii (Fig. 18b). We use a different number of stars per bin for the middle and bottom panels. The overall trend of the $\sigma_{v}$ is an initial decline followed by a flat or rising profile. Similar profile behaviors up to and beyond the nominal King radius are also seen in the velocity dispersion profiles of Ursa Minor (Muñoz et al. 2005), Draco (Muñoz et al. 2005), and Sculptor (Westfall et al. 2006). As discussed in these references, such behavior means either that we are seeing evidence for tidal disruption in these dSphs, or that these satellites have very extended DM components dynamically traced by these stars at large radii.

Closer inspection of the top panels of Figure 18 shows a number of stars (more at larger radius) that have an RV deviating significantly from the mean. These outliers ${ }^{25}$ are predominantly toward the high RV side and create an obvious asymmetric tail in the distribution of Leo I velocities shown in Figure 14. Indeed, as shown in Figure 19, it appears that these outliers create a secondary hump in the RV distribution about $20 \mathrm{~km} \mathrm{~s}^{-1}$ higher than the Leo I mean RV of $288.8 \mathrm{~km} \mathrm{~s}^{-1}$, but in the least there is an obvious asymmetric tail toward larger RVs.

Members of the outlier population we identify as those stars with RVs beyond $2.3 \sigma$ (where $\sigma$ refers to the velocity dispersions of the entire sample, i.e., $8.9 \mathrm{~km} \mathrm{~s}^{-1}$ ) for the Leo I RV sample with $r_{\text {maj }} / r_{\text {lim }}<0.6$ and those with RVs beyond $1.5 \sigma$ for $r_{\text {maj }} / r_{\text {lim }} \geq 0.6$ Leo I stars. Our tighter criterion for the stars at larger radii is based on examination of the RV distribution shown in Figure 19: the tight peak in the $r_{\text {maj }} / r_{\text {lim }}>0.6$ distribution clearly stands out from a broader distribution of RVs and is well approximated by a Gaussian of dispersion of $3.9 \mathrm{~km} \mathrm{~s}^{-1}$. Because this tight peak is centered on the mean velocity of the Leo I core, it seems appropriate to associate it with the typical RV population at smaller radii (although the latter has a broader distribution). But it

25 Although we use the term outliers, these are obviously associated with Leo I. 

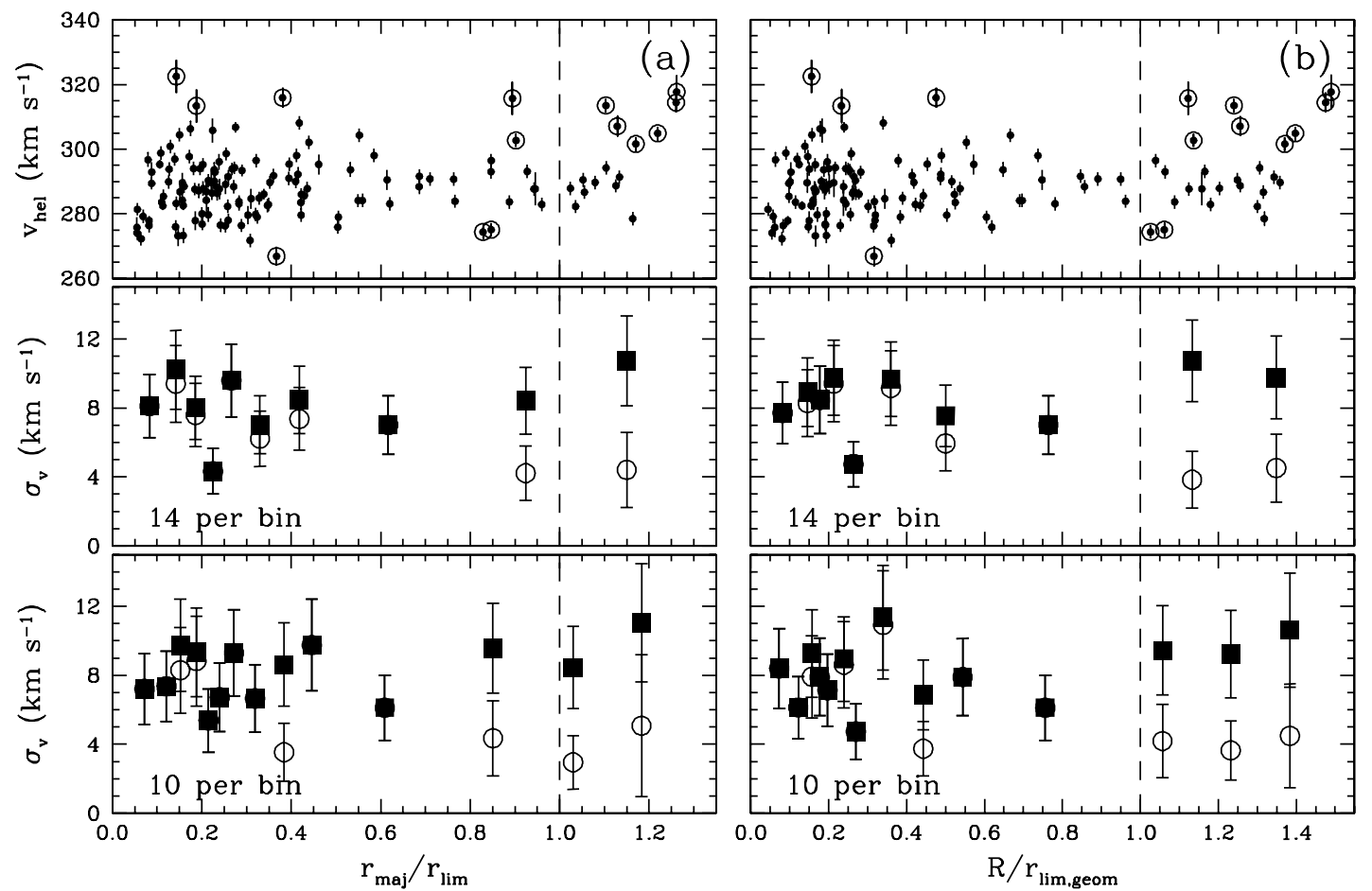

FIG. 18. - Radial variation of individual RVs and velocity dispersions using $(a)$ elliptical and $(b)$ circular radii. The number of stars for calculating each data point $\left(\sigma_{v}\right)$ in the middle panels is 14 , except for the last data point where 11 stars were used. Similarly, 10 stars were used for calculating each data point in the bottom panels, except for the last data point where seven stars were used. The vertical dashed lines denote the King limiting radius [geometric mean, i.e., $r_{\text {lim,geom }}=r_{\text {lim }}(1-e)^{1 / 2}$ for the right panels]. The RVoutliers (see text) are marked with large circles in the top panels, and velocity dispersions computed without the outliers are shown with open circles in the middle and bottom panels.

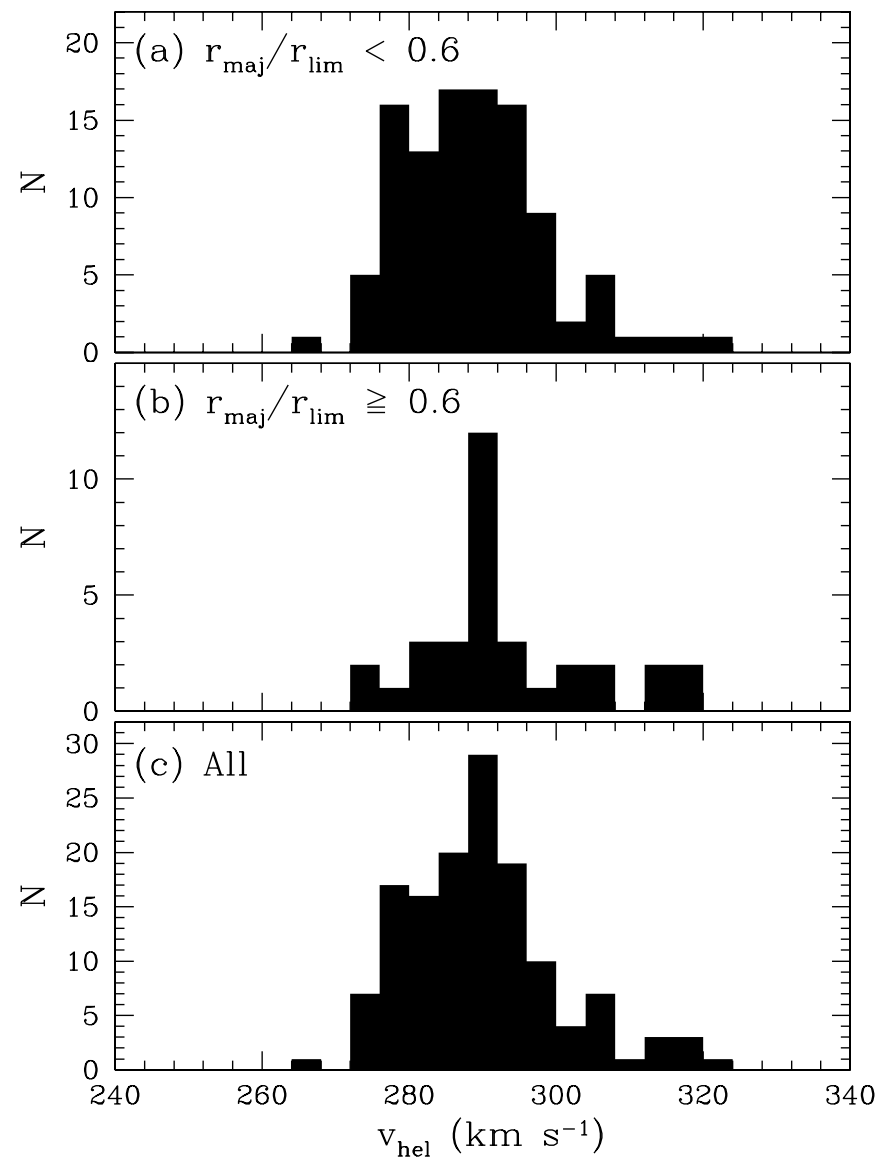

FIG. 19.- - Histogram for heliocentric RVs of (a) Leo I giants with $r_{\text {maj }} / r_{\text {lim }}<$ $0.6,(b)$ Leo I giants with $r_{\text {maj }} / r_{\text {lim }} \geq 0.6$, and $(c)$ all Leo I giants. is apparent that about half of the outer Leo I stars are part of a second, much more broadly RV-distributed, outlier population in Figure $19 b$. Adopting a $3 \sigma$ cut based on the rather cold, $3.9 \mathrm{~km} \mathrm{~s}^{-1}$ central dispersion peak among the stars with $r_{\text {maj }} / r_{\text {lim }}>0.6$ identifies the same set of RVoutliers as a $1.5 \sigma$ scheme where the entire Leo I sample $\sigma$ is used.

The outliers so identified are marked with open circles in the top panels of Figure 18. The spatial distribution of these stars is shown in Figure 20 with respect to the entire sample of Leo I giant candidates, where it may be seen that not only do they tend to lie to the west side of Leo I, but they are predominantly found near or beyond the nominal King radius. The latter point is not simply due to the combined facts that we have used a tighter "outlier" definition for stars at large radii and that all of the stars in the western DEIMOS mask are at large radii: In fact, there are still 11 stars to the east side of Leo I center with $r_{\text {maj }} / r_{\text {lim }} \geq 0.6$, but only one of these is an outlier by the above definition, compared to nine outliers among $22 r_{\text {maj }} / r_{\text {lim }} \geq 0.6$ stars on the west side. Of the nine outliers to the west, eight are outliers to the positive RV side.

Exclusion of these outliers from the calculation of $\sigma_{v}$ trends leads to different results, as shown by open circles in the middle and bottom panels of Figure 18: the velocity dispersion tends to fall with radius, and to a relatively cold value at large radius. For the stars beyond the King limiting radius, the dynamically cold population has a mean $\mathrm{RV}$ of $287.7 \pm 1.6 \mathrm{~km} \mathrm{~s}^{-1}$, close to the systemic velocity of the Leo I core, and a velocity dispersion of $4.0 \pm 1.6 \mathrm{~km} \mathrm{~s}^{-1}$. These dynamically cold stars also appear to be more in line with the Leo I major axis, as seen in Figure 20. The RV outlier stars, when included, increase the dispersion outside the limiting radius to $10.3 \pm 2.2 \mathrm{~km} \mathrm{~s}^{-1}$.

Both the presence and meaning of velocity dispersion "cold points" near the nominal King radii of various dSphs have been 


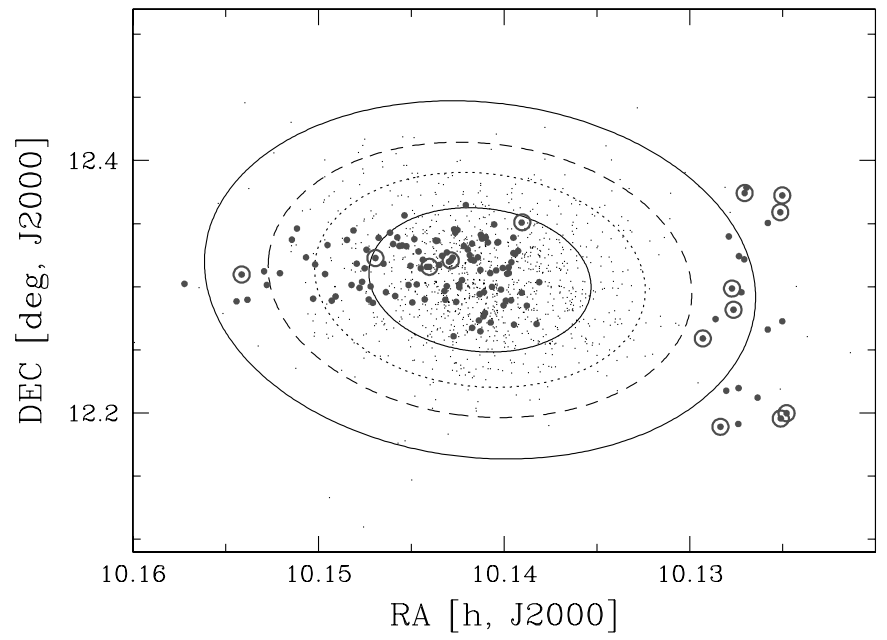

FIG. 20.- Same as Fig. 16, but for the RV outliers in Fig. 18 marked as open circles. The dotted ellipse corresponds to $r_{\mathrm{maj}} / r_{\mathrm{lim}}=0.6$. [See the electronic edition of the Journal for a color version of this figure.]

debated recently (Wilkinson et al. 2004; Lokas et al. 2005; Muñoz et al. 2005). The reality of the phenomenon turns on how one deals with (i.e., includes or excludes) apparent outliers and how one bins the data (Łokas et al. 2005; Muñoz et al. 2005). In the present case, where the Leo I systemic velocity is so extreme, it is hard to believe that the outliers are anything but Leo I- associated stars, and so we are presented with a situation where the outliers and the dispersion characteristics demand an explanation within the context of a dSph structural model.

The lopsided nature of the RV outliers (i.e., that they tend to be outliers to the high RV side) provides a significant clue to the nature of the outer parts of the Leo I dSph. Were the RV outliers bound stars within a large Leo I DM halo, it is hard to understand why they would not exhibit symmetrical dynamics, i.e., a hot population of stars with members both approaching and receding relative to the Leo I core. The lack of any particular spatial concentration of the RV outliers (other than tending to the west side of Leo I) does not support the idea that the RV asymmetry is caused by a star cluster or "dynamical fossil" like that recently suggested to be "sloshing back and forth within the" DM halo of the Ursa Minor dSph (Kleyna et al. 2003). The observation of significant spatial "substructure" within dSphs (e.g., Olszewski \& Aaronson 1985; Demers et al. 1995; Eskridge \& Schweitzer 2001; Kleyna et al. 2003; Palma et al. 2003; Coleman et al. 2004; Coleman \& Da Costa 2005; Olszewski et al. 2006) has long been a concern for DM models, where high DM densities should quickly smooth such substructure out. Here we are presented with an apparent substructure evident both dynamically and spatially.

A more natural explanation of the RV distribution is that the RV outliers and many of the stars outside the nominal King limiting radius represent stars that have likely been tidally stripped from Leo I, whereas the more dynamically cold component at the nominal Leo I systemic velocity seen at large radii represents the outermost reaches of the bound population of Leo I stars. The decreasing RV dispersion trend when the outliers are excluded suggests that we are seeing the tapering gravitational field of a Leo I apparently lacking an extended DM halo. Moreover, the observed asymmetric RV distribution is naturally produced by tidal debris, as we show in $\S 6.2$. A spectroscopic survey over a larger area would of course help clarify the dynamics of the outer Leo I system: in particular, spectroscopic observations at large radius on the eastern side of Leo I would provide particularly strong leverage on the tidal debris model, which would predict negative RV outliers to the east side of Leo I.

\section{DISCUSSION}

\subsection{Dark Matter, Extended Halos, and Tidal Stripping}

The question of the origin of the apparently large $M / L$ in dSph galaxies remains one of the most vexing in Local Group research. While the presence of large amounts of DM is the most popular explanation (e.g., M98), other proposals, including modified Newtonian dynamics (MOND; see Sanders \& McGaugh 2002 and references therein), tidal heating and/or disruption, dynamical resonances (Kuhn \& Miller 1989), and even the notion that dSphs are completely unbound (Kroupa 1997; Klessen \& Kroupa 1998), have been proposed to explain the large velocity dispersions observed in the cores of the Galactic dSphs. Leo I provides an interesting opportunity to revisit some of these mechanisms because its large galactocentric distance and velocity imply that Leo I could only have had a few encounters with the MW center. Tidal heating/disruption or resonance effects, if they occur, will have been limited to those that could organize and be sustained over only one or two perigalactica. Because of this, Leo I offers the unique chance to gauge the importance of such effects on a per perigalacticon basis.

\subsubsection{The Case for Tidal Disruption}

In $\S 4.2$ we have rederived the $M / L$ of Leo I using standard core-fitting prescriptions and the central velocity dispersion. The results are not much different than previous findings, which suggest a modest DM component in Leo I compared to that in other dSphs. A primary point of this paper is to establish whether, independent of the net DM content of the dSph, it may have extended structure indicating that it is being tidally disrupted.

We believe the weight of evidence suggests compellingly that Leo I has indeed been tidally disrupted and, moreover, that the derived King limiting radius approximates the true tidal radius in this particular system:

1. We find a significant number of widely placed giant candidates associated with Leo I in our survey area (see Fig. 7) and with an especially pronounced density near the King limiting radius. While it might be argued that these stars represent a secondary, bound "halo" population around Leo I, perhaps tracing an extended DM halo, as can be seen in Figure 8, the break population stars are more spread out along the east-west direction than in north-south in a manner that resembles tidal arms. In order to demonstrate the "bipolarity" of these outer stars more clearly, we divide the region outside the break radius of Leo I into azimuthal sectors of $18^{\circ}$ in width and $17^{\prime}$ in major-axis radial length $\left(r_{\text {maj }}=10.4^{\prime}-27.5^{\prime}\right)$ and count the giant candidates in each sector. ${ }^{26}$ The counts were then normalized by the surveyed area in each sector to obtain the mean densities as a function of

\footnotetext{
${ }^{26}$ Careful examination of the stellar distribution in model galaxies for tidal disruption models shows that stars released during the most recent perigalactic pass form a tidal tail-like feature mostly aligned with the semimajor axes of the satellite, whereas those that become unbound during older perigalactic passes tend to be spread out irrespective of the semimajor axes. One demonstration can be found in Fig. 23, an orbital plot of our $\mathrm{N}$-body simulations where we use different colors for stars that become unbound during different perigalactic passes. Consequently, photometrically chosen $\mathrm{dSph}$ candidates located on degree scales away from the satellite's main body will not necessarily show a clearly defined tidal tail-like configuration. For this reason, we have limited our sample in $r_{\text {maj }}$ when constructing the azimuthal sector count plot.
} 


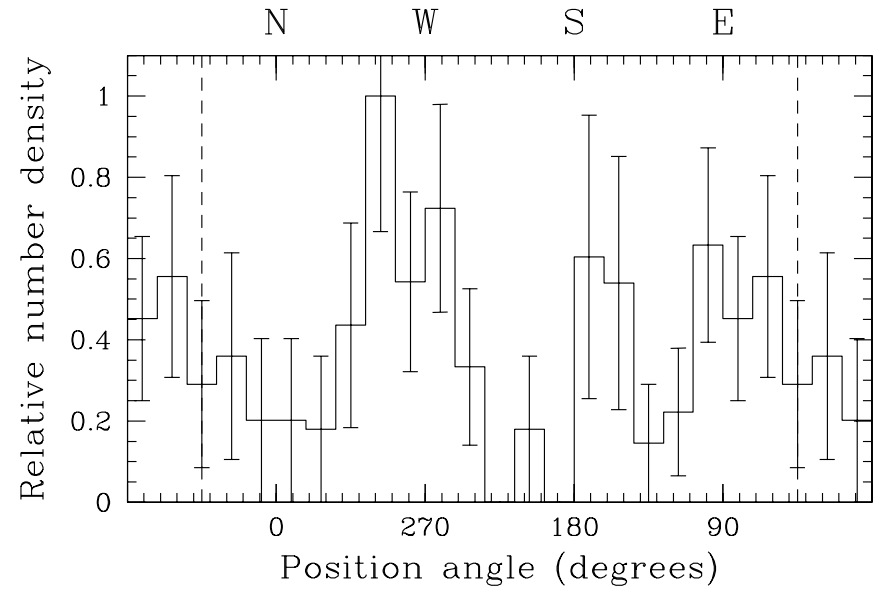

FIG. 21.-Normalized number densities of "extratidal" stars as a function of azimuthal sectors $\left(18^{\circ}\right.$ wide) around Leo I. The histogram represents the number density in each elliptical sector outside the break radius (major-axis radial distance of $10.2^{\prime}$; see Fig. 8 ). The final results were divided by the maximum number density to scale the relative number densities from 0 to 1 . The repeated dashed line at P.A. $=45^{\circ}$ is added for guidance. The broad peaks to the east and west reflect apparent tidal arms stretching out from the main body. The excess of density at $180^{\circ}-120^{\circ}$ is due to the "bridge" of stars that extends to the southeast and is likely a statistical anomaly.

azimuthal sector presented in Figure 21. As may be seen, the "arms" are represented by two broad density peaks separated by $\sim 180^{\circ}$ to the east and west. A minimum in density is apparent to the north, but a corresponding minimum to the south is interrupted by the presence of a small peak at $145^{\circ}-180^{\circ}$ that originates from the bridge of seven stars extending to the southeast (visible in Fig. 8). Whether this feature is a chance alignment of Leo I stars or a real dynamical structure may require highresolution velocity data to resolve, but we note that if these stars were a bit more spread out in position angle, the "minimum" of "beyond the break" stars to the south would look similar to that of the north. Overall, the stars beyond the break radius appear to be spread out more along the major axis than the minor axis and have the appearance of a nascent tidal tail system. It is difficult to understand how the observed increasing ellipticity of the system would originate and be sustained in a dSph with an extended DM halo; a very elongated luminous halo population with anisotropic velocity ellipsoid structure overlapping and extending beyond a second, more concentrated population with a rounder distribution would be implied.

2. Our "King+break" radial density profile for Leo I is similar to the profiles observed in previous models of disrupting satellites (Johnston et al. 1999, 2002; see also our new $N$-body simulation presented in $\S 6.2$ ), as well as in the Sagittarius dSph (Majewski et al. 2003), an acknowledged example of a tidally disrupting satellite.

3. We have found a flat overall velocity dispersion profile for Leo I, a profile that is expected in the case of a tidally disrupting dSph (Kroupa 1997; R. R. Muñoz et al. 2007, in preparation) and is actually observed in the case of the tidally disrupting Sgr dSph (Majewski et al. 2004). While such profiles could also be formed in the presence of an extended DM halo (Kleyna et al. 2001), the detailed velocity distribution at large radius is not what is expected for the latter model, whereas it compellingly resembles (Fig. 19) a dynamically cold (likely bound) stellar population at the systemic Leo I velocity with a superposed population of unbound stars with larger velocity dispersion.

4. The asymmetry of the RVs of the outliers would seem to challenge the bound, extended halo population hypothesis, since in this model stars at a variety of orbital phases (i.e., both approaching and leaving the apocenters of their internal orbits) would be expected to yield a symmetrical velocity distribution. On the other hand, such asymmetries are not only accommodated but expected in tidal tail models (as we show in $\S 6.2$ ).

We conclude that the most straightforward and natural interpretation for the observed Leo I structure and dynamics is that (1) it is tidally limited near the observed King limiting radius with the dwindling bound population creating a dynamically cold signature at its outermost extent, and (2) increasing numbers of unbound stars are being observed at larger radius and forming nascent tidal tails that contribute the RV outliers at large radius. If this proposed model for the structure of Leo I is true, we may infer several things immediately about the mass-loss history and orbit of this satellite galaxy, even without resorting to new $N$-body simulations.

\subsubsection{Inferred Mass-Loss Rate}

Models of tidally disturbed satellites around the MW by Johnston et al. $(1999,2002)$ predict breaks in satellite radial density profiles similar to that shown in Figure $9 b$. Under the assumption that Leo I stars found past the radial density profile break are unbound, extratidal debris, we may estimate the massloss rate. Johnston et al. (1999) provide an algorithm for determining the approximate mass-loss rate of a satellite using the strength of the break population. If the density profile of a satellite galaxy shows a break at $r_{\text {break }}$ and the extrabreak population is well defined out to a radius of $r_{\mathrm{xt}}$, the fractional mass-loss rate can be estimated from

$$
\left(\frac{d f}{d t}\right)_{1}=g(\theta) \frac{r_{\text {break }}}{r_{\mathrm{xt}}-r_{\text {break }}} \frac{n_{\mathrm{xt}}}{n_{\text {break }}} \frac{\pi}{T_{\text {orb }}},
$$

where $\theta$ is the angle between the line of sight and the plane perpendicular to the satellite velocity, $g(\theta)$ is a geometric factor corresponding to the orbit (which can be approximated as $\cos \theta), n_{\mathrm{xt}}$ is the number of extratidal stars between $r_{\text {break }}$ and $r_{\mathrm{xt}}$, $n_{\text {break }}$ is the number of stars within $r_{\text {break }}$, and $T_{\text {orb }}$ is the period for a circular orbit at the present satellite galactocentric distance. For now we adopt the values of $\theta$ and $T_{\text {orb }}$ for Leo I listed in Table 4 of Johnston et al. (1999) (but provide a new model for this orbit in $\S 6.2$ ). By examining the radial density profile in Figure $9 b$, we determine $r_{\text {break }}=10.2^{\prime}$. For $r_{\mathrm{xt}}$, we have adopted the majoraxis radial distance of the farthest data point in Figure $9 b$ since the corresponding surface density is near the background level. Applying corrections to account for missing catalog giant stars falling in the Mosaic CCD chip gaps gives $n_{\text {break }}=1150$. For the extratidal count, we scale our counts of stars at each annulus by the ratio of the total elliptical area to the amount of that annulus in our actual field coverage, and we find $n_{\mathrm{xt}}=129$. Using these values, we obtain a mass-loss rate of $(d f / d t)_{1}=3.1 \times 10^{-3} \mathrm{Gyr}^{-1}$, which implies that an average of less than $1 \%$ of the total mass of Leo I is lost per gigayear. We note that the mass-loss rate equation (3) above is technically for satellites with extratidal radial density profiles that follow $\Sigma_{\mathrm{xt}} \sim r^{-1}$. In our case, the measured outer density slopes are steeper than $\gamma=-1$ (see Fig. 9b). Johnston et al. (1999) also note that this equation is only good to within a factor of 2. Therefore, the combined uncertainty in the mass-loss estimation is likely to be large. Nevertheless, the small net massloss rate for Leo I is consistent with the upper limit determined by Johnston et al. (1999) although our new density profile of Leo I has allowed us to recalculate this improved upper limit to the mass-loss rate. 
For satellites with a less well-defined extratidal population, Johnston et al. (1999) derived an expression for estimating the upper limit of the fractional mass-loss rate, and using more realistic models, Johnston et al. (2002) update the relation to

$$
\left(\frac{d f}{d t}\right)_{2}=\frac{2}{5} \frac{\Sigma_{\mathrm{xt}}\left(r_{\text {break }}\right)}{n_{\text {break }}} \frac{\pi}{T_{\text {circ }}} 2 \pi r_{\text {break }}^{2}
$$

where $\Sigma_{\mathrm{xt}}\left(r_{\text {break }}\right)$ is the number density at $r=r_{\text {break }}$ and $T_{\text {circ }}$ is same as $T_{\text {orb}}$. Using the values for the repeated parameters from above, we obtain an upper limit of $(d f / d t)_{2}=5.1 \times 10^{-2} \mathrm{Gyr}^{-1}$, similar to what Johnston et al. (1999) found.

Our results confirm the results of Johnston et al. (1999) that among the dSphs studied by IH95, Leo I apparently has one of the smallest mass-loss rates. The large orbit and few perigalactic passes of Leo I may explain this low mass-loss rate. For example, Leo II, which has a lower RV at a similar distance to Leo I (which implies a smaller apogalacticon), has a higher estimated mass-loss rate. Despite the relatively low fractional mass-loss rate derived for Leo I, that it has a perceptible one at all, and so clearly in the form of tidal tails, shows that no MW dSph with a smaller orbit and mass (i.e., virtually all other known Galactic dSphs) is likely to be immune from significant tidal effects (Johnston et al. 2002).

\subsection{A Tidally Disrupting Satellite Model to Explain the Outer Leo I Structure and Dynamics}

\subsubsection{Fitting a Leo I Model}

Johnston et al. (2002) have $N$-body simulated a number of examples of disrupting satellite galaxies in MW-like potentials, including one system with mass and orbital properties likely similar to Leo I (their models 4 and 5), and show how tidal disruption can be expected even in extreme cases of a satellite in a large orbit like that of Leo I. Here we undertake additional modeling specifically guided by the new Leo I observations to see whether we can obtain a somewhat closer model match to the newly observed Leo I structure and velocities found here. Our primary goal is to understand the nature of the extended population observed in the Leo I profile. More specifically, we seek to test whether a tidal disruption scenario can explain the observed (1) elongated dSph structure at radii comparable to and larger than the limiting radius (i.e., the bipolar distribution of the break population), (2) shape of the overall density profile, (3) dispersion profile over all radii, and (4) asymmetric RV distribution. A disrupting model that simultaneously accounts for all or even some of these features would not only lend support to a tidal disruption scenario but (as we show below) also provide the first real constraints on the orbit of Leo I.

The $N$-body simulation code we adopt has the same heritage as the Johnston et al. (2002) mass-follows-light (i.e., single component) models. The static MW potential has a logarithmic, spherical $(q=1.0)$ halo with circular speed $210 \mathrm{~km} \mathrm{~s}^{-1}$. The assumed solar distance to the Galactic center is $8.5 \mathrm{kpc}$, and the total Galactic mass within $50 \mathrm{kpc}$ is $4.5 \times 10^{11} M_{\odot}$. Other aspects of the potential are as in Law et al. (2005).

The satellite is modeled by $10^{5}$ particles originally configured as a Plummer (1911) model,

$$
\Phi=-\frac{G M_{\text {satellite }, 0}}{\sqrt{r^{2}+r_{0}^{2}}},
$$

which has a physical scale length parameter, $r_{0}$. The model is constrained so that the present satellite position and RV match those observed for Leo I: $(l, b)=\left(226^{\circ},+49^{\circ}\right)$, a $259 \mathrm{kpc}$ heliocentric distance, and galactocentric velocity $\left(v_{\mathrm{GSR}}\right)$ of $180 \mathrm{~km} \mathrm{~s}^{-1}$. However, because the proper motion of Leo I is unknown, this is a free parameter that ultimately determines the shape of the model Leo I orbit. After assuming a given present proper motion, the Leo I orbit (with the satellite as a point mass) is evolved back in time long enough to derive the phase-space position of the satellite two apogalactica ago. The point mass is then replaced by the Plummer model satellite at this phase-space position (after the satellite has been allowed to evolve and relax at infinite distance), and a full $\mathrm{N}$-body simulation is evolved forward to the present time. In general, for the models tested here the start of the simulations occurs about 11-12 Gyr ago. In the course of the modeling efforts for Leo I, some 100 differently configured models have been run.

Initial models were run to fix a likely orientation of the orbit, under the assumption that the east-west position angle of the Leo I ellipticity and the orientation of the break population are caused by tidal effects and tidal stripping, respectively. $\mathrm{N}$-body simulations were run with the satellite having an orbital pole every $45^{\circ}$ along its allowed set of poles (its "great circle pole family;" see Palma et al. 2002). It was found that a satellite with orbital pole near $(l, b)=\left(122^{\circ},+13^{\circ}\right)$ yielded tails with the proper orientation, although this simulation cannot be discriminated (on the basis of the direction of Leo I's stretching alone) from one with a satellite having an opposite orbital pole [i.e., $\left(302^{\circ},-13^{\circ}\right)$ ]. We adopt either of these antipodal orbital poles for the remaining simulations, which thereby fixes the position angle of the present Leo I proper motion to $\sim 75^{\circ}$ or $\sim 255^{\circ}$ in Galactic coordinates.

The remaining simulation variables we explore are the initial satellite mass and scale $\left(r_{0}\right)$, which determine the size and density of the satellite and therefore the degree of satellite disruption, and the magnitude of the proper motion, which determines the properties (eccentricity, perigalacticon, and apogalacticon) of the orbit and also affects the degree of satellite disruption. Given the extreme distance and RV of Leo I, it seems probable that Leo I has a rather elliptical orbit (Taylor et al. 2004b). Thus, our modeling efforts centered on orbits with perigalactica ranging from 10 to $50 \mathrm{kpc}$ (and consistent with the observed RV of Leo I); despite the large variation in perigalactica, this actually corresponds to orbital eccentricities ranging only from 0.80 to 0.96 . With such orbits Leo I has an orbital period of about $6 \mathrm{Gyr}$. It is found that after fixing the satellite mass to of order that found in $\S 4.2$, a scale of $r_{0}=0.3 \pm 0.1 \mathrm{kpc}$ yields a final Leo I satellite with a tidal radius of order the observed Leo I King limiting radius. Thus, we adopt models with this general structure and explore how varying the orbit shape is reflected in the resultant radial light profile and velocity distribution. For the latter, we "observe" RVs sampled from the model distribution in a spatial "footprint" mimicking that of the Keck DEIMOS spectra as shown in Figure 16; this gives the most direct and fair comparison to the observed RV distribution seen in Figures 14 and 18.

It is found that a variety of simulations of Leo I on an eccentric orbit can reproduce not only the overall "King+break" radial light profile that is characteristic of disrupting satellites (Johnston 1998) but also an asymmetric RV distribution and a more or less flat velocity dispersion profile like that observed. To fine-tune the model, we took account of three general correlations in turn (see R. R. Muñoz et al. 2007, in preparation): (1) The central velocity dispersion directly reflects the adopted satellite mass. (2) The size of the observed "King profile" part of the satellite is set by the initial Plummer model scale $r_{0}$. (3) With mass and scale set by the previous conditions, the mass-loss rate (hence the size of the break population) is then only driven by the orbital shape 

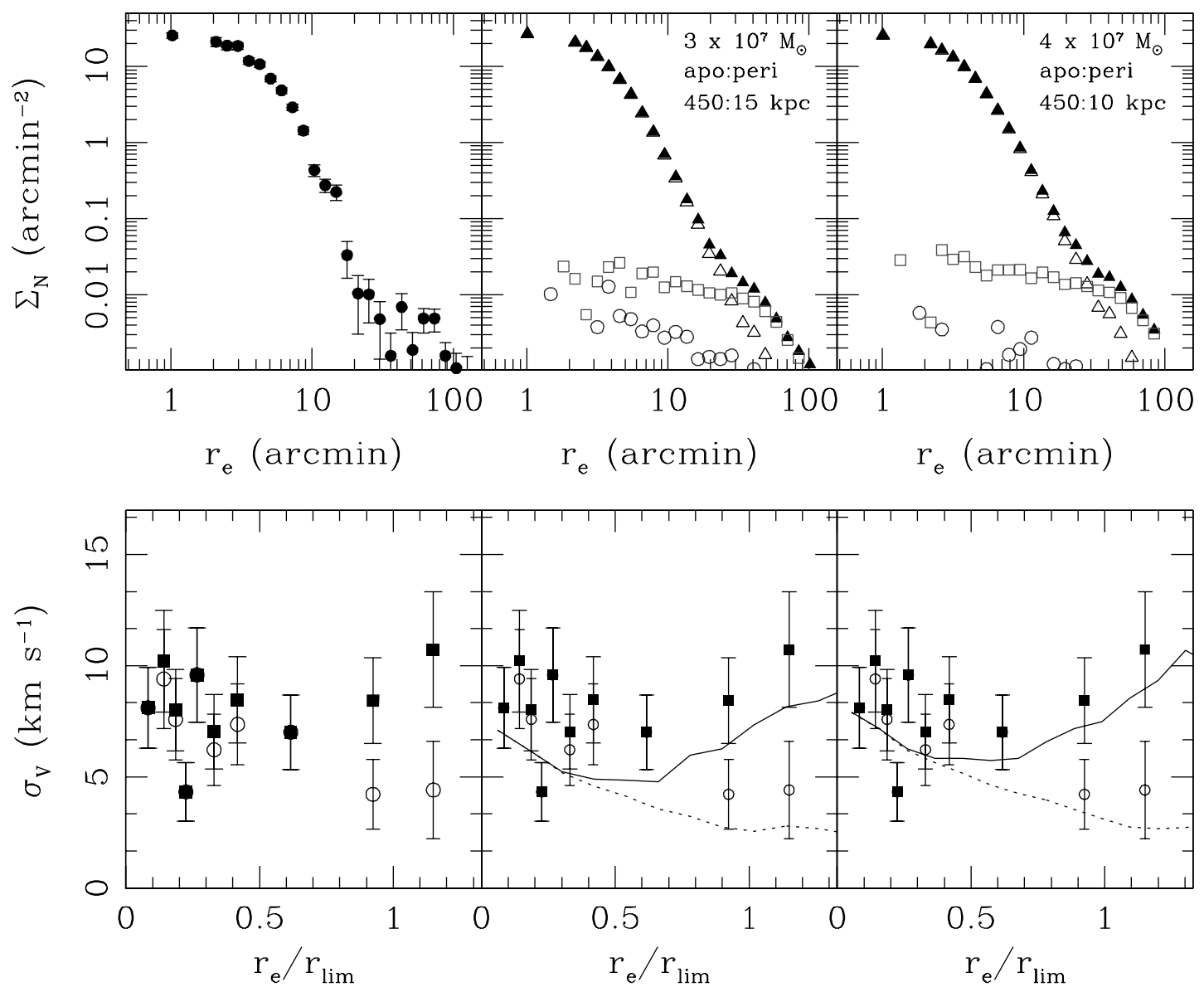

FIG. 22.-Comparisons of observed (left panels) surface radial density profile (top panels) and velocity dispersion profile (bottom panels) with those of $N$-body models. The filled triangles in the model surface density profiles are for the entire sample, while open triangles are for the bound stars. The open circles and open squares in the top panels are for unbound stars from the first and second mass-loss events, respectively. The symbols used for the velocity dispersion profiles are the same as those in the middle panel of Fig. 18a, while the solid and dashed lines show the model velocity dispersion profiles for the entire sample and the bound stars, respectively. [See the electronic edition of the Journal for a color version of this figure.]

(i.e., impact parameter to the MW). Following these general guides, a narrow set of mass, scale, and orbital shapes were found to give reasonable matches to the observed Leo I properties. The density and RV properties of two of these "matching" models are compared against the data in Figure 22, and their threedimensional orbits are shown in Figure 23.

The parameters and results for adopted models are listed in Table 5. Models 111 and 117 have been run in the MW potential for 12.0 and $11.8 \mathrm{Gyr}$, respectively. In both cases the net mass loss is modest, with only $16 \%$ and $23 \%$ of the initial mass being unbound after 12 Gyr. We note that the implied mass-loss rates for both models roughly match the estimates made in $\S 6.1 .2$. As found in previously published simulations of satellite disruption (e.g., Law et al. 2005), the break populations in the extreme orbit, Leo I model satellite density profiles are also found to be due to tidal disruption; this is shown in Figure 22, where stars that are still bound and those that have been lost and become unbound on each of the last two radial orbits are marked with separate symbols. Thus, our original supposition that the observed Leo I break population may be due to unbound stars is supported by the models. However, the relative density at which these breaks occur does not match that of the Leo I density profile if the beginning of the unbound debris is associated with the inner of the two radial profile breaks. Said another way, these models provide a good match to all the Leo I data if the first break in the radial density profile is considered as statistically insignificant or structurally unrelated to unbound tidal debris. Even if the latter suppositions are not valid, we contend that these models still provide as good or a better simultaneous match to the overall observed structure and dynamics of any specific dSph than has been offered before.

We also attempted to find a model that could accommodate the inner density law break. Since the relative density at which tidally induced break populations occur is directly related to the instantaneous rate at which debris is being generated, in order to match the relative position compared to the central density of the inner break at higher density, we need to increase the mass-loss rate of the satellite. This can be done by decreasing the satellite density. Figure 24 shows the properties of model 122, which has an initial mass of $6.5 \times 10^{7} M_{\odot}$, a scale of $0.55 \mathrm{kpc}$, and apo: perigalacticon of $450: 10 \mathrm{kpc}$. Whereas the position of the model break is now matched to the inner of the two observed density breaks, the model satellite is found to be 3 times larger than the actual Leo I, and the velocity dispersion and the overall density profile are no longer a good match. We therefore find model 122 to provide a less satisfactory description of Leo I than models 111 and 117 .

The final satellite masses for models 111 and 117 (see Table 5) are consistent with the Leo I mass derived from core fitting in $\S 4.2$ within the errors. The implied current total Leo I massto-light ratio from the models is $M / L=3.1-4.5 M_{\odot} L_{\odot}^{-1}$. This lower $M / L$ than found in $\S 4.2$ implies a relatively small DM content and would bring Leo I in line with the $M / L$ typical of $\mathrm{dE}$ 

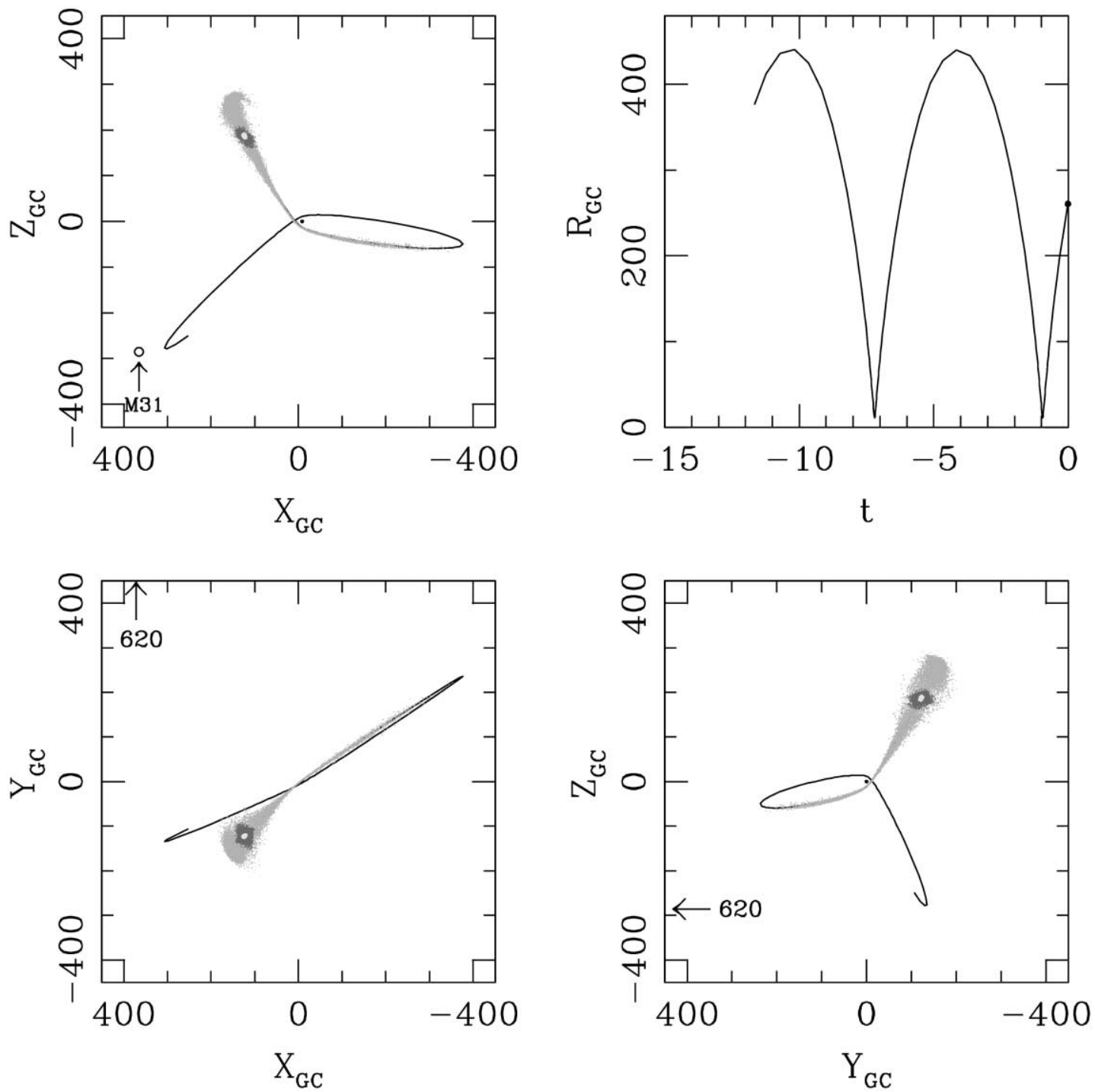

FIG. 23.-Orbital properties of model 117. This orbit is very similar to the one for model 111 since the primary difference between them is the closer perigalacticon distance in model 117. White dots represent particles that are still bound. Dark gray and light gray mark particles that became unbound in the first and second perigalacticon passages, respectively. The current position of M31 is indicated as arrows. [See the electronic edition of the Journal for a color version of this figure.]

galaxies and even globular clusters. It is important to recognize that we have obtained a good match to the Leo I RV asymmetry with both of the preferred models even though this observed property was not used as a model constraint. That such a result comes naturally lends further confidence to the plausibility of our models. The RV asymmetry in the models arises from the long extension of the trailing tidal arms toward the inner Galaxy (and the observer) seen in Figure 23 and for which the innermost parts have a significant projection along the line of sight in models 111 and 117. However, the degree of that projection, and

TABLE 5

Parameters and Results for Adopted Models

\begin{tabular}{|c|c|c|c|c|c|c|c|c|}
\hline Model & $\begin{array}{c}m_{i}^{\mathrm{a}} \\
\left(10^{7} M_{\odot}\right)\end{array}$ & $\begin{array}{c}r_{0}^{\mathrm{b}} \\
(\mathrm{kpc})\end{array}$ & $\begin{array}{l}r_{\text {peri }}{ }^{\mathrm{c}} \\
(\mathrm{kpc})\end{array}$ & $\begin{array}{l}r_{\text {apo }}{ }^{\mathrm{d}} \\
(\mathrm{kpc})\end{array}$ & $\begin{array}{c}P^{\mathrm{e}} \\
(\mathrm{Gyr})\end{array}$ & $\begin{array}{l}T_{\text {peri }}{ }^{\mathrm{f}} \\
\text { (Gyr) }\end{array}$ & $\begin{array}{c}d f / d t^{\mathrm{g}} \\
\left(10^{-2} \mathrm{Gyr}^{-1}\right)\end{array}$ & $\begin{array}{c}m_{f}^{\mathrm{h}} \\
\left(10^{7} M_{\odot}\right)\end{array}$ \\
\hline $111 \ldots \ldots \ldots$ & 3.0 & 0.3 & 15 & 450 & 6.33 & 0.96 & 1.3 & 2.3 \\
\hline $117 \ldots \ldots \ldots \ldots \ldots \ldots \ldots \ldots \ldots \ldots$ & 4.0 & 0.3 & 10 & 450 & 6.05 & 0.95 & 2.2 & 3.4 \\
\hline
\end{tabular}

${ }^{\mathrm{a}}$ Initial mass of model satellite.

b Scale length parameter in eq. (5).

c Perigalactic distance.

d Apogalactic distance.

e Radial period.

f Time since last closest approach.

g Fractional mass-loss rate.

${ }^{\mathrm{h}}$ Final mass of model satellite. 

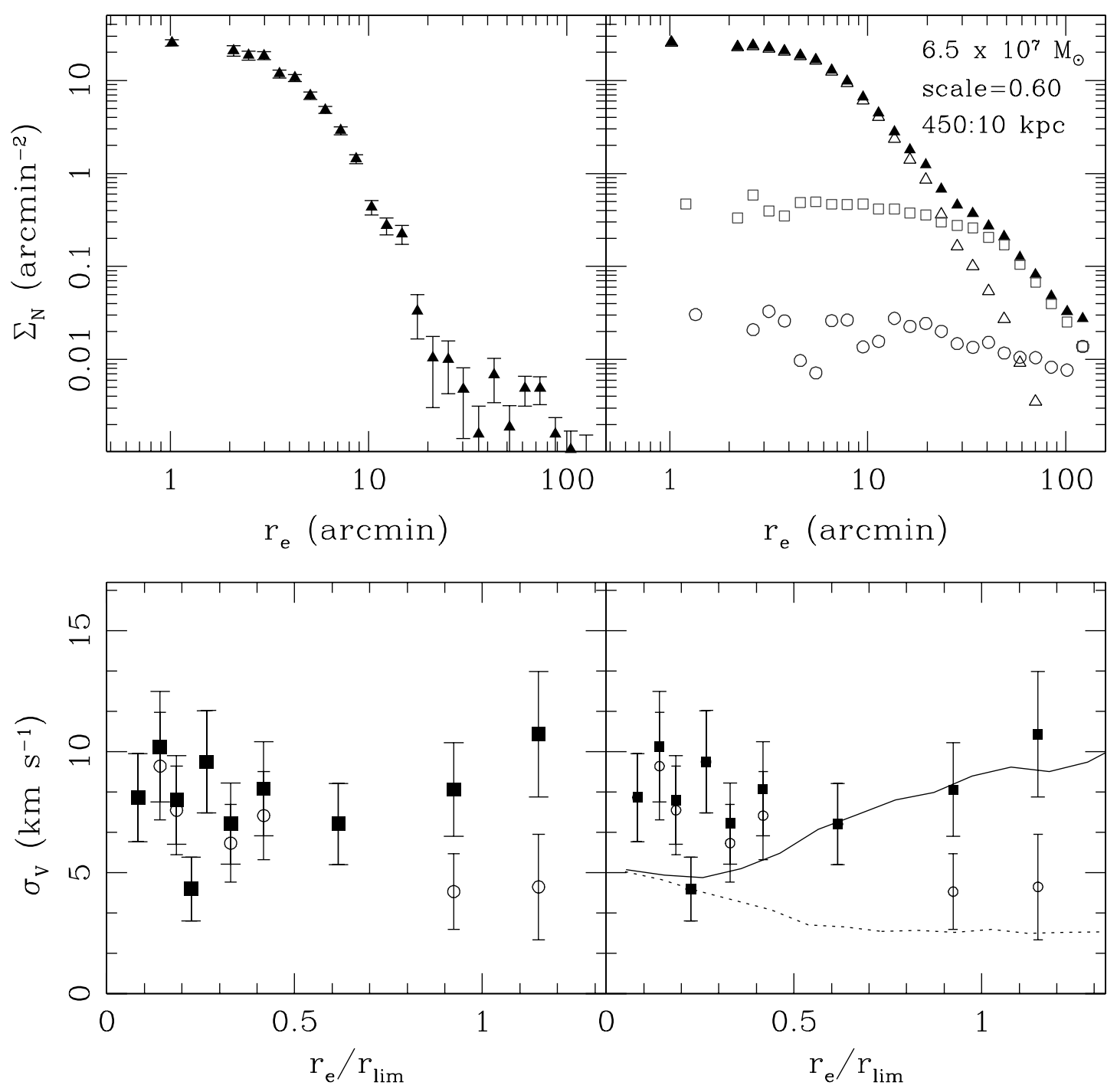

Fig. 24.-Comparisons of observed (left panels) surface radial density profile (top panels) and velocity dispersion profile (bottom panels) with those of model 122 . The symbols and lines are the same as those in Fig. 22. [See the electronic edition of the Journal for a color version of this figure.]

therefore the velocity spread of the asymmetry, is obviously tied with the eccentricity of the orbit. This can be seen, for example, by the results (Fig. 25) of a series of models run with the same mass and scale, but more circular orbits than shown in Figure 23. As Figure 25 shows, less eccentric orbits have less asymmetry in their observed RV distribution. In addition, as might be expected, these models give poorer matches to the radial density and velocity dispersion profiles. In this way, the observed RV distribution can apparently directly constrain the detailed shape of the Leo I orbit, and by direct comparison to the observed RV distribution, we deduce that Leo I has an orbital eccentricity similar to that shown in Figure 23. We also deduce the general direction of the model $111 / 117$ orbits (i.e., the general direction of the proper motion) to be correct (i.e., to the east), since a satellite with a similar eccentricity orbit but opposite orbital pole yields the opposite RV asymmetry for our Keck spectroscopy mask footprint (Fig. 16). This is demonstrated by the model results shown in the rightmost panel of Figure 25.

Finally, because we have shown by the models that stars in the break population are well described as nascent tidal tails, and by the analysis in $\S 6.1 .1$ we have shown that the Leo I break population lies predominantly east-west, we conclude that the orbital pole of $(l, b)=\left(122^{\circ},+13^{\circ}\right)$ is approximately correct.
Thus, based on the apparent discriminatory power of the Figure 22 parameters and the direction of the tidal arms, we conclude that the orbit shown in Figure 23 is a reasonable approximation to the true Leo I orbit. A check on the orbit will obviously be delivered by the measurement of the Leo I proper motion, which can be expected in a decade or so from a key project (led by SRM) of NASA's Space Interferometry Mission PlanetQuest. The expected current proper motions for Leo I are predicted to be $\left(\mu_{l} \cos b, \mu_{b}\right)=(0.0046,0.0219)$ and $(0.0020,0.0138) \mathrm{mas} \mathrm{yr}^{-1}$, respectively, for models 111 and 117 (including solar motion, which is assumed to have a $232 \mathrm{~km} \mathrm{~s}^{-1}$ revolutionary speed about the MW, and additional peculiar motion of 9.0 and $7.0 \mathrm{~km} \mathrm{~s}^{-1}$ in the Galactic radial and $Z$ directions, respectively).

In the meantime, verification of the overall picture painted by our model fitting here, including the orbit, can be made by obtaining more RVs on the east side of Leo I. Our models predict that at large radii on the east side of Leo I an asymmetry of the RV distribution should be observed opposite (i.e., toward lower $\mathrm{RVs}$ ) that we have found to the west. The change in the sense of asymmetry arises from the sampling of the leading arm of the Leo I tidal tails on the east side, whereas our western RVs have been sampling trailing arm debris. Mapping the Leo I tidal tails to larger angular separations would also provide significant leverage 


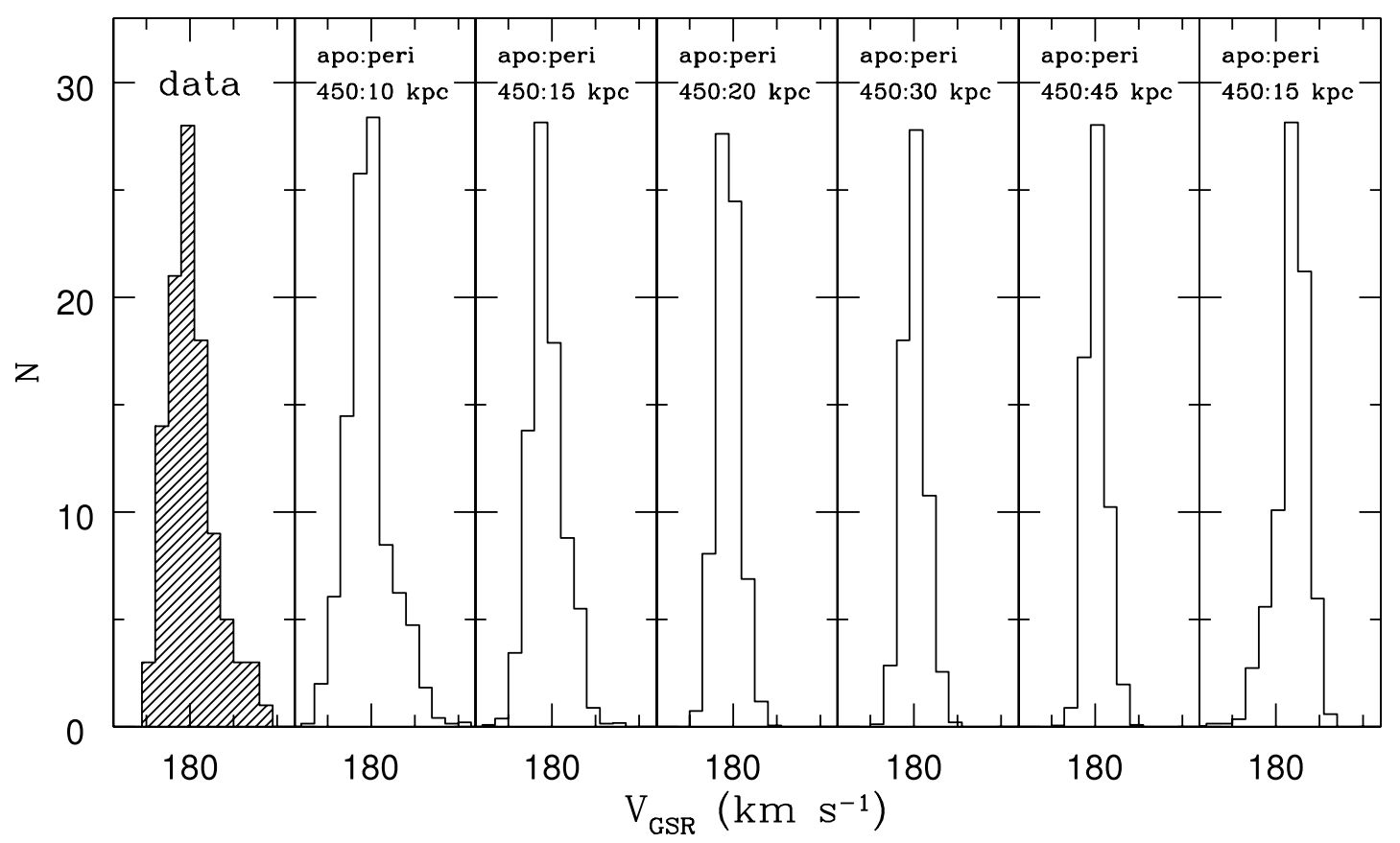

FIG. 25.-Observed galactocentric RV distribution (leftmost panel) compared with the model velocity distributions using the DEIMOS footprint for Leo I. In each model panel, the apogalacticon and perigalacticon distances are noted. The rightmost panel is for a model with opposite orbital pole.

on the Leo I orbit and mass-loss history. Figure 23 suggests that quite long tails should exist for two orbits of mass loss. However, the problem is quite observationally challenging because the tails are at fairly low surface brightness and the corresponding densities of Leo I tidal tail giant stars will eventually become quite sparse. A search for the MSTO CMD feature for the Leo I tidal arms near the satellite will require reaching to $V>28$ over large areas. From the ground, such work is severely hampered by the difficulty of star/galaxy separation at these magnitudes (D. Martinez-Delgado 2006, private communication).

\subsubsection{Implications for the Local Group Path of Leo I and Mass of the Galaxy}

The provenance of Leo I has important implications for the mass of the MW. The high RV of Leo I at $259 \mathrm{kpc}$ translates to a large implied lower limit to the escape velocity of the MW if this satellite is bound. If Leo I has made two orbits about the MW, as assumed in our models, then it must be bound. If it has made only one perigalactic passage, Leo I could still be bound to the MW, having become so on the last orbit; however, in this case Leo I may also be unbound and on a hyperbolic orbit.

Unfortunately, it is not clear that we can, with certainty, establish whether two orbits or only one have occurred. According to the models, much of the unbound debris observed near Leo I has detached in the last orbit (see Fig. 23). To verify whether any differences can be discerned between a one- and two-orbit Leo I, we ran a simulation (model 118) with the same satellite and orbital properties as model 111, but where only one radial orbit has occurred (i.e., the model is started 4.3 Gyr ago). Figure 26 shows that the radial density profile from such a simulation is virtually indistinguishable from the two-orbit model 111. This is because of the small contribution of older debris to the density of Leo I over the currently observed area. On the other hand, this small contribution does become more obvious in its influence on the observed velocity dispersion, as seen in the bottom panels of Figure 26: The one-orbit model has a smaller velocity dispersion at large radii compared to that of the two-orbit model, where older debris helps inflate the dispersion. The latter, two-orbit dispersion profile is a closer match to that observed for Leo I, and so, based on this evidence alone, we can tentatively suggest that a two-orbit model is favored for Leo I.

The star formation history of Leo I may lend circumstantial support to the two-orbit scenario. Models 111 and 117 suggest that Leo I endured fairly substantial tidal shocking both $\sim 1$ and $\sim 7.5$ Gyr ago. This general orbital picture seems to coincide with the star formation history of Leo I studied by Gallart et al. (1999a), who find that most of its star formation activity occurred between 7 and 1 Gyr ago. The oldest ( $>10$ Gyr) Leo I population, discovered by Held et al. (2000; blue HB stars) and Held et al. (2001; RR Lyrae variables), likely formed as a result of the initial collapse of gas that led to the formation of Leo I. The "major" star formation that started $\sim 7$ Gyr ago is roughly timed with the previous perigalactic passage in our model (see also models by Mayer et al. 2001). We can only speculate as to the causes of the abrupt drop in star formation at $\sim 1$ Gyr ago. Leo I may have simply ran out of gas to fuel the star formation. The absence of gas in Leo ${ }^{27}$ (Knapp et al. 1978; Bowen et al. 1997) is consistent with this picture. The coincidence that Leo I passed through the inner Galaxy about this time (and possibly with a rather small impact parameter; Fig. 23) may also suggest gas stripping by the MW disk or perhaps by a dense high-velocity cloud. A massive $(3 \times$ $10^{6} M_{\odot}$ ) fragmented $\mathrm{H}$ I cloud structure around Leo I (Blitz \& Robishaw 2000) perhaps indicates that the gas has been perturbed by tidal shocking. Nevertheless, something triggered star formation at a time that matches well the timing of the first perigalacticon in our two-orbit model, and, together with the velocity dispersion data, our results support the hypothesis of Z89 that Leo I is bound to the MW and has had two radial orbits in this state.

If Leo I is bound, a large MW mass is implied. The total mass of the MW within $260 \mathrm{kpc}$ from the center in our simulations

\footnotetext{
27 Blitz \& Robishaw (2000) find extended H I emission around Leo I but not toward the galaxy itself.
} 

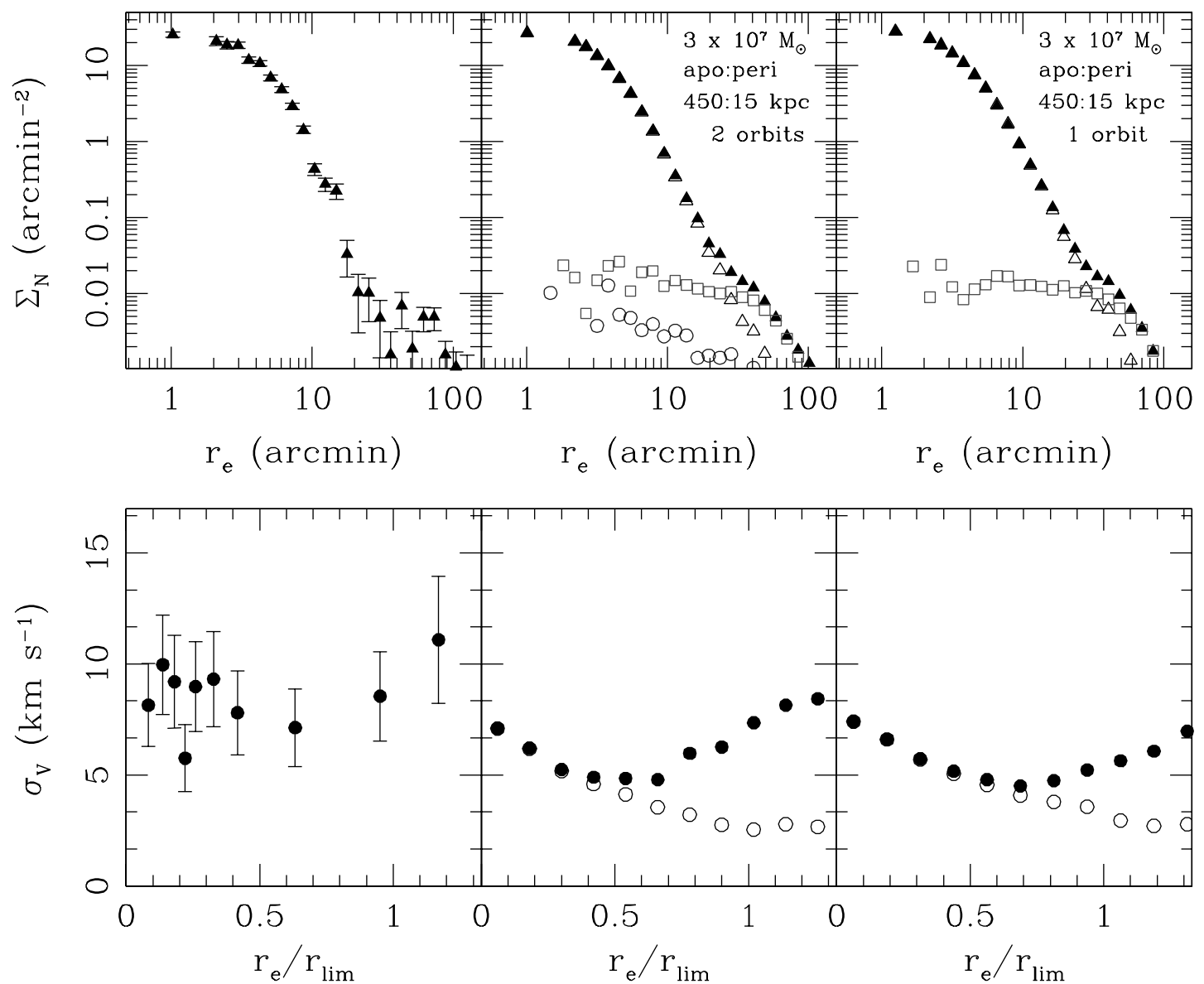

FIG. 26.-Comparisons of observed (left panel) surface density profile and velocity dispersion profile with those of models having the same orbital and structural

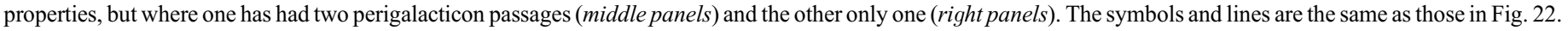
[See the electronic edition of the Journal for a color version of this figure.]

is $1.8 \times 10^{12} M_{\odot}$, and it has a profile yielding a mass interior to $50 \mathrm{kpc}$ that is consistent with those found in the analyses by Z89, Wilkinson \& Evans (1999), and Sakamoto et al. (2003) when these analyses include Leo I as a bound satellite. Verifying the length of the Leo I tidal tails would verify whether it has had multiple orbits around the MW and is thus bound. Obviously, a definitively measured proper motion for Leo I would also help determine the true orbit of Leo I and refine the mass of our Galaxy.

What do our results say about the origin of Leo I? First, it is probable that a hyperbolic orbit for Leo I would produce similar results (over the sky area we have surveyed here) to those seen for a single-orbit, bound model. Moreover, such an orbit will have an overall shape not dissimilar in overall orientation and general direction to the last orbit of models 111 and 117, and thus we may determine from which direction Leo I approached the MW in this case. Interestingly, in either the one-orbit (bound or hyperbolic) or two-orbit case, our modeling seems to rule out any close association of Leo I with M31 over relevant timescales to tidal stripping: (1) In the bound case, Figure 23 shows that Leo I is not in the vicinity of M31 [which has current MW coordinates of $\left.(X, Y, Z)_{\mathrm{GC}} \sim(375,620,-285) \mathrm{kpc}\right]$ since approximately two orbital periods ago, and even then the inferred distance between M31 and Leo I is $\sim 700 \mathrm{kpc}$ (and this ignores the motion of M31 over a Hubble time!). (2) In the hyperbolic case, Leo I would have apparently entered the Local Group in an orbital plane almost perpendicular to the direction of M31 (i.e., from the general direction of the last apogalactica shown in Fig. 23). Were the Byrd et al. (1994) hypothesis that Leo I was once bound to M31 to be true, it would have had to have been released from M31 at least $\sim 10$ Gyr ago.

Finally, we add a note of caution about interpreting the potential shape of the Leo I orbit as inferred or extrapolated from Figure 23, which represents a model run in a static MW potential. Obviously, currently favored hierarchical galaxy formation models imply a continuing growth of the Galactic potential with time, and this will alter the orbits of satellites. On the other hand, the MW formation models of Bullock \& Johnston (2005) suggest that destroyed satellites contributing mass to the MW halo accrete predominantly before the last two postulated Leo I perigalactica (i.e., before 7 Gyr ago). Thus, at least over this timescale, a static MW approximation and the resulting Figure 23 orbit may be appropriate descriptors. Moreover, as recently shown by Peñarrubia et al. (2006), tidal streams respond adiabatically to evolving potentials, so that even if the MW potential evolved over the past 7 Gyr or so, the Leo I tidal stream visible today would resemble that derived in a static MW potential with the present-day mass profile.

\section{SUMMARY AND CONCLUSIONS}

We have photometrically surveyed a $4.5 \mathrm{deg}^{2}$ region centered on Leo I in the $M, T_{2}$, DDO51 filter system in order to explore the extended morphology of this dwarf spheroidal galaxy, which is currently regarded as the most distant of the known Galactic satellites (unless the more distant Phoenix system is bound to the MW). 
The photometric data were used to select Leo I giant candidates based on the two criteria: (1) the gravity-sensitive ( $M$ - DDO51, $M-T_{2}$ ) diagram, which separates distant giant stars from contaminating, foreground, metal-rich disk dwarfs, and (2) CMD positions commensurate with the temperature-apparent magnitude combination of stars on the Leo I giant branch. The background level of our "Leo I giant star" sample is determined to be small, and a $100 \%$ reliability in the identification of bona fide Leo I giants is found via testing with a total of 133 stars in the Leo I field with previously published or new Keck spectroscopy.

We derive a new set of Leo I structural parameters by fitting a single-component King profile to the Leo I giant candidates. Coupling this to the central velocity dispersion we have measured (§ 5.5), we use core-fitting techniques to derive a mass for the Leo I system of $3.5 \times 10^{7} M_{\odot}$ and a total $(M / L)_{V}$ of $4.6 M_{\odot} L_{\odot, V}^{-1},{ }^{28}$ values not too dissimilar from previous study using the same technique (M98). The two-dimensional distribution of Leo I giant candidates shows many giant stars outside the derived King limiting radius. These are primarily along the major axis, and spectroscopy of a subsample of these "extratidal" stars shows that they are actually associated with the $\mathrm{dSph}$. This population of stars shows up as a break at a major-axis radial distance of $\sim 10^{\prime}$ in the radial density profile. Our new Keck spectroscopy confirms this excess of stars beyond the King limiting radius to be made of true Leo I members.

Our Leo I velocity dispersion profile shows a flat and then rising trend at large radii. We also find that the Leo I RV distribution, particularly for stars at large angular separations (which in our data are primarily to the west side of Leo I), includes a population of stars with a broad and skewed, asymmetrical distribution toward positive RVs overlapping a second, very much colder population at the nominal Leo I mean velocity.

We interpret these features as support for a picture wherein Leo I has been tidally disrupted on at least one, but at most two, perigalactic passages by a massive Local Group member (most likely the MW for both) and find these phenomena naturally produced in mass-follows-light $N$-body simulations of a tidally disrupting Leo I analog in an MW-like potential. The bestmatching $N$-body simulations to both the observed structural morphology and velocity distribution of Leo I are those where the tidally disrupting satellite is on a rather high eccentricity $(0.93-$ $0.96)$, small perigalacticon $(10-15 \mathrm{kpc})$, bound orbit around the MW and has a present total $M / L \sim 3-4.5 M_{\odot} L_{\odot}^{-1}$. These bestfitting model masses are $58 \%-85 \%$ the $M / L$ derived from the central velocity dispersion and core fitting, but it is not unreasonable to presume that the latter method yields somewhat inflated masses because of systematic increases in the true dispersion due to astrophysical processes such as the presence of binaries and the atmospheric jitter common to giant star atmospheres. Thus, the likely $M / L$ of the satellite is rather modest and not unlike those of other elliptical systems of approximately similar mass scale that are typically regarded as low in, or devoid of, DM (dE galaxies and globular clusters).

Because of the rather close match between our model results and observations, and because disrupting satellites on highly radial orbits appear to yield great discriminatory power in this regard, we have been able to constrain the likely orbit of Leo I without the measurement of its proper motion. The orientation of the current satellite orbital plane can be fixed by matching model tidal tails to the predominant direction of the observed break population (i.e., more or less along the major axis), whereas we

\footnotetext{
28 These values are $14 \%$ smaller than those in $\S 4.2$, where the M98 central velocity dispersion was used to derive the mass.
}

find that the direction of angular momentum in this orbital plane is well constrained by the sense of the RV asymmetry we have observed to the west side of the satellite. Our models demonstrate that a positive RV asymmetry in the models is produced by trailing tidal debris for the receding satellite. Thus, we predict that an opposite RV asymmetry will be found on the east side of Leo I from leading tidal debris. Such a result would provide an important verification of the tidal disruption model we have put forward in this paper.

Our observed Leo I RV distribution is most consistent with a two MW orbit history for Leo I, with both orbits around the MW; however, we cannot yet definitively rule out a one-orbit scenario. However, whether Leo I is bound to the MW or on an unbound, hyperbolic orbit around the MW, our results seem to rule out a Leo I orbit that includes a previous association with M31 within the last $10 \mathrm{Gyr}$, in contradistinction to the Byrd et al. (1994) scenario of a relatively recent origin of Leo I from M31.

Leo I has long played a "spoiler" role in setting the mass of the MW because of its huge $r v^{2}$ lever arm in Jeans equationbased determinations using tracer particles of unknown proper motion (i.e., unknown orbit size and shape). The large implied escape velocity at Leo I's distance implies large MW masses if Leo I is bound to our Galaxy (Z89; Wilkinson \& Evans 1999), although its influence on the mass determination is lessened when more complete samples of objects with complete phase-space data are employed (e.g., Sakamoto et al. 2003). Our observed RV distribution for Leo I slightly favors a two perigalacticon pass, bound MW orbit for Leo I, thereby suggesting that higher mass estimates for the MW may be more correct.

In general, our tidally disrupting mass-follows-light satellite models provide a quite satisfactory match to the observed properties of Leo I, but a few details, namely, the apparent double break in the density profile and the density at which the inner of these breaks occurs, we have yet to account for in these initial modeling efforts. Nevertheless, we contend that the scenario of a tidally disrupting, low- $M / L$ system on a highly radial orbit provides a rather complete explanation for the observed properties of Leo I. While some properties of Leo I and other dSphs (e.g., flat velocity dispersion profiles and break populations) have also been explained by postulating that these systems are embedded in extended DM halos, such an explanation in the case of Leo I appears less compelling in that it cannot account for as many of the observed properties of the system (e.g., the asymmetry in the $\mathrm{RV}$ distribution and increasing ellipticity with radius). In contrast, tidal tails by now provide a well-established observational paradigm for dSph satellites, with the Sagittarius $\mathrm{dSph}$ the most vivid example. We contend that the tidal disruption of Sagittarius is not unique and that Leo I may be another example of the phenomenon, albeit at a much lower mass-loss rate commensurate with a satellite on such an extreme orbit. Tidal disruption observed among both the closest and farthest of the MW satellites suggests that this process may be ubiquitous, and that similarly structured satellites with elliptical orbits and distances between these two examples might also be expected to be experiencing tidally induced mass loss, likely with an intermediary range of mass-loss rates.

The authors wish to thank Chris Palma for providing several useful computer programs and Alison Coil for aiding the Keck DEIMOS observations. The referee has provided a number of useful comments that have improved the paper. Support for this 
work at Virginia was provided by NSF grants AST 97-02521 and AST 03-07851, a Cottrell Scholar Award from the Research Corporation, NASA/JPL contract 1228235, the David and Lucile Packard Foundation, and the generous support of The F. H. Levinson Fund of the Peninsula Community Foundation. K. V. J.'s contribution was supported through NASA grant NAG5-9064 and NSF CAREER award AST 01-33617. M. H. S. received thesis travel support from KPNO that was used during the collection of the Mosaic data and support from the STScI Director's Discretionary Research Fund.
Armandroff, T. E., \& Da Costa, G. S. 1986, AJ, 92, 777

Armandroff, T. E., Olszewski, E. W., \& Pryor, C. 1995, AJ, 110, 2131

Azzopardi, M., Lequeux, J., \& Westerlund, B. E. 1985, A\&A, 144, 388 1986, A\&A, 161, 232

Bellazzini, M., Gennari, N., Ferraro, F. R., \& Sollima, A. 2004, MNRAS, 354, 708

Belokurov, V., Evans, N. W., Irwin, M. J., Hewett, P. C., \& Wilkinson, M. I. 2006a, ApJ, 637, L29

Belokurov, V., et al. 2006b, ApJ, 642, L137

Benson, A. J., Lacey, C. G., Baugh, C. M., Cole, S., \& Frenk, C. S. 2002, MNRAS, 333, 156

Binney, J., \& Tremaine, S. 1987, Galactic Dynamics (Princeton: Princeton Univ. Press)

Blitz, L., \& Robishaw, T. 2000, ApJ, 541, 675

Bowen, D. V., Tolstoy, E., Ferrara, A., Blades, J. C., \& Brinks, E. 1997, ApJ, 478, 530

Bullock, J. S., \& Johnston, K. V. 2005, ApJ, 635, 931

Bullock, J. S., Kravtsov, A. V., \& Weinberg, D. H. 2000, ApJ, 539, 517 2001, ApJ, 548, 33

Byrd, G., Valtonen, M., McCall, M., \& Innanen, K. 1994, AJ, 107, 2055

Caputo, F., Cassisi, S., Castellani, M., Marconi, G., \& Santolamazza, P. 1999, AJ, 117, 2199

Chen, B., et al. 2001, ApJ, 553, 184

Christlieb, N., Green, P. J., Wisotzki, L., \& Reimers, D. 2001, A\&A, 375, 366

Coleman, M. G., \& Da Costa, G. S. 2005, Publ. Astron. Soc. Australia, 22, 162

Coleman, M. G., Da Costa, G. S., Bland-Hawthorn, J., Martínez-Delgado, D., Freeman, K., C., \& Malin, D. 2004, AJ, 127, 832

Côté, P., Mateo, M., Sargent, W. L. W., \& Olszewski, E. W. 2000, ApJ, 537, L91

Crane, J. D., Majewski, S. R., Rocha-Pinto, H. J., Frinchaboy, P. M., Skrutskie,

M. F., \& Law, D. R. 2003, ApJ, 594, L119

Cseresnjes, P. 2001, A\&A, 375, 909

Davis, M., Efstathiou, G., Frenk, C. S., \& White, S. D. M. 1985, ApJ, 292, 371

Demers, S., \& Battinelli, P. 2002, AJ, 123, 238

Demers, S., Battinelli, P., Irwin, M. J., \& Kunkel, W. E. 1995, MNRAS, 274, 491

Demers, S., Irwin, M. J., \& Gambu, I. 1994, MNRAS, 266, 7

Eskridge, P. B. 1988a, AJ, 95, 1706 1988b, AJ, 96, 1352

Eskridge, P. B., \& Schweitzer, A. E. 2001, AJ, 122, 3106

Evans, N. W., \& Wilkinson, M. I. 2000, MNRAS, 316, 929

Evans, N. W., Wilkinson, M. I., Guhathakurta, P., Grebel, E. K., \& Vogt, S. S. 2000, ApJ, 540, L9

Faber, S. M., et al. 2003, Proc. SPIE, 4841, 1657

Fox, M. F., \& Pritchet, C. J. 1987, AJ, 93, 1381

Fusi Pecci, F., Bellazzini, M., Cacciari, C., \& Ferraro, F. R. 1995, AJ, 110, 1664

Gallart, C., Freedman, W. L., Aparicio, A., Bertelli, G., \& Chiosi, C. 1999a, AJ, 118,2245

Gallart, C., et al. 1999b, ApJ, 514, 665

Geehan, J. J., Fardal, M. A., Babul, A., \& Guhathakurta, P. 2006, MNRAS, 366, 996

Geisler, D. 1990, PASP, 102, 344

1996, AJ, 111, 480

Gerhard, O. E., \& Spergel, D. N. 1992, ApJ, 389, L9

Goerdt, T., Moore, B., Read, J. I., Stadel, J., \& Zemp, M. 2006, MNRAS, 368, 1073

Gómez-Flechoso, M. Á., \& Martínez-Delgado, D. 2003, ApJ, 586, L123

Gould, A., Guhathakurta, P., Richstone, D., \& Flynn, C. 1992, ApJ, 388, 345

Grillmair, C. J., \& Dionatos, O. 2006, ApJ, 641, L37

Grillmair, C. J., \& Johnson, R. 2006, ApJ, 639, L17

Harrington, R. G., \& Wilson, A. G. 1950, PASP, 62, 118

Hayashi, E., Navarro, J. F., Taylor, J. E., Stadel, J., \& Quinn, T. 2003, ApJ, 584, 541

Held, E. V., Clementini, G., Rizzi, L., Momany, Y., Saviane, I., \& Di Fabrizio, L. 2001, ApJ, 562, L39

Held, E. V., Saviane, I., Momany, Y., \& Carraro, G. 2000, ApJ, 530, L85

Hinkle, K., Wallace, L., Valenti, J., \& Harmer, D., eds. 2000, Visible and Near Infrared Atlas of the Arcturus Spectrum 3727-9300 Å (San Francisco: ASP)

Hodge, P. W. 1963, AJ, 68, 470

. 1971, ARA\&A, 9, 35

\section{EFERENCES}

Ibata, R. A., Gilmore, G., \& Irwin, M. J. 1995, MNRAS, 277, 781 Illingworth, G. 1976, ApJ, 204, 73

Irwin, M., \& Hatzidimitriou, D. 1995, MNRAS, 277, 1354 (IH95)

Johnston, K. V. 1998, ApJ, 495, 297

Johnston, K. V., Choi, P. I., \& Guhathakurta, P. 2002, AJ, 124, 127

Johnston, K. V., Sigurdsson, S., \& Hernquist, L. 1999, MNRAS, 302, 771

Johnston, K. V., Spergel, D. N., \& Hernquist, L. 1995, ApJ, 451, 598

Kang, X., Mao, S., Gao, L., \& Jing, Y. P. 2005, A\&A, 437, 383

Kauffmann, G., White, S. D. M., \& Guiderdoni, B. 1993, MNRAS, 264, 201

King, I. R. 1962, AJ, 67, 471 1966, AJ, 71, 64

Klessen, R. S., \& Kroupa, P. 1998, ApJ, 498, 143

Kleyna, J. T., Geller, M. J., Kenyon, S. J., Kurtz, M. J., \& Thorstensen, J. R. 1998, AJ, 115, 2359

Kleyna, J. T., Wilkinson, M. I., Evans, N. W., \& Gilmore, G. 2001, ApJ, 563, L115

Kleyna, J. T., Wilkinson, M. I., Evans, N. W., Gilmore, G., \& Frayn, C. 2002, MNRAS, 330, 792

Kleyna, J. T., Wilkinson, M. I., Gilmore, G., \& Evans, N. W. 2003, ApJ, 588, L21 Klypin, A., Kravtsov, A. V., Valenzuela, O., \& Prada, F. 1999, ApJ, 522, 82

Knapp, G. R., Kerr, F. J., \& Bowers, P. F. 1978, AJ, 83, 360

Kroupa, P. 1997, NewA, 2, 139

Kroupa, P., Theis, C., \& Boily, C. M. 2005, A\&A, 431, 517

Kuhn, J. R., \& Miller, R. H. 1989, ApJ, 341, L41

Kuhn, J. R., Smith, H. A., \& Hawley, S. L. 1996, ApJ, 469, L93

Kunkel, W. E. 1979, ApJ, 228, 718

Kunkel, W. E., Irwin, M. J., \& Demers, S. 1997, A\&AS, 122, 463

Law, D. R., Johnston, K. V., \& Majewski, S. R. 2005, ApJ, 619, 807

Lee, K. H., Lee, H. M., Fahlman, G. G., \& Sung, H. 2004, AJ, 128, 2838

Lee, M. G., Freedman, W., Mateo, M., Thompson, I., Roth, M., \& Ruiz, M.-T. 1993, AJ, 106, 1420

Lejeune, T., \& Buser, R. 1996, Baltic Astron., 5, 399

Libeskind, N. I., Cole, S., Frenk, C. S., Okamoto, T., \& Jenkins, A. 2007, MNRAS, 374, 16

Libeskind, N. I., Frenk, C. S., Cole, S., Helly, J. C., Jenkins, A., Navarro, J. F., \& Power, C. 2005, MNRAS, 363, 146

Lin, D. N. C., \& Faber, S. M. 1983, ApJ, 266, L21

Łokas, E. L. 2002, MNRAS, 333, 697

Łokas, E. L., Mamon, G. A., \& Prada, F. 2005, MNRAS, 363, 918

Lynden-Bell, D. 1982, Observatory, 102, 202

Lynden-Bell, D., \& Lynden-Bell, R. M. 1995, MNRAS, 275, 429

Majewski, S. R. 1994, ApJ, 431, L17

Majewski, S. R., Law, D. R., Polak, A. A., \& Patterson, R. J. 2006, ApJ, 637, L25

Majewski, S. R., Ostheimer, J. C., Kunkel, W. E., \& Patterson, R. J. 2000a, AJ, 120,2550

Majewski, S. R., Ostheimer, J. C., Patterson, R. J., Kunkel, W. E., Johnston, K. V., \& Geisler, D. 2000b, AJ, 119, 760

Majewski, S. R., Skrutskie, M. F., Weinberg, M. D., \& Ostheimer, J. C. 2003, ApJ, 599, 1082

Majewski, S. R., et al. 2004, AJ, 128, 245

. 2005, AJ, 130, 2677

Mateo, M., Olszewski, E. W., Vogt, S. S., \& Keane, M. J. 1998, AJ, 116, 2315 (M98)

Mateo, M. L. 1998, ARA\&A, 36, 435

Mayer, L., Governato, F., Colpi, M., Moore, B., Quinn, T., Wadsley, J., Stadel, J., \& Lake, G. 2001, ApJ, 559, 754

Monet, D., et al. 1998, USNO-A2.0: A Catalog of Astrometric Standards (Annapolis: USNO)

Moore, B. 1996, ApJ, 461, L13

Moore, B., Ghigna, S., Governato, F., Lake, G., Quinn, T., Stadel, J., \& Tozzi, P. 1999, ApJ, 524, L19

Moore, B., Governato, F., Quinn, T., Stadel, J., \& Lake, G. 1998, ApJ, 499, L5

Morrison, H. L., Mateo, M., Olszewski, E. W., Harding, P., Dohn-Palmer, R. C., Freeman, K. C., Norris, J. E., \& Morita, M. 2000, AJ, 119, 2254

Muñoz, R. R., et al. 2005, ApJ, 631, L137

2006, ApJ, 649, 201

Navarro, J. F. 2004, in IAU Symp. 220, Dark Matter in Galaxies, ed. S. D. Ryder (San Francisco: ASP), 61 
Navarro, J. F., Frenk, C. S., \& White, S. D. M. 1996, ApJ, 462, 563 1997, ApJ, 490, 493

Newberg, H. J., et al. 2002, ApJ, 569, 245

Odenkirchen, M., et al. 2001, ApJ, 548, L165 2003, AJ, 126, 2385

Oh, K. S., Lin, D. N. C., \& Aarseth, S. J. 1995, ApJ, 442, 142

Olszewski, E. W., \& Aaronson, M. 1985, AJ, 90, 2221

Olszewski, E. W., Mateo, M., Harris, J., Walker, M. G., Coleman, M. G., \& Da Costa, G. S. 2006, AJ, 131, 912

Ostheimer, J. C. 2002, Ph.D. thesis, Univ. Virginia

Palma, C., Majewski, S. R., \& Johnston, K. V. 2002, ApJ, 564, 736

Palma, C., Majewski, S. R., Siegel, M. H., Patterson, R. J., Ostheimer, J. C., \& Link, R. 2003, AJ, 125, 1352

Paltoglou, G., \& Bell, R. A. 1994, MNRAS, 268, 793

Peñarrubia, J., Benson, A. J., Martínez-Delgado, D., \& Rix, H. W. 2006, ApJ, 645,240

Piatek, S., \& Pryor, C. 1995, AJ, 109, 1071

Plummer, H. C. 1911, MNRAS, 71, 460

Pritzl, B. J., Armandroff, T. E., Jacoby, G. H., \& Da Costa, G. S. 2002, AJ, 124, 1464

Reid, N., \& Majewski, S. R. 1993, ApJ, 409, 635

Reid, N., \& Mould, J. 1991, AJ, 101, 1299

Rocha-Pinto, H. J., Majewski, S. R., Skrutskie, M. F., \& Crane, J. D. 2003, ApJ, 594, L115

Rockosi, C. M., et al. 2002, AJ, 124, 349

Sakamoto, T., Chiba, M., \& Beers, T. C. 2003, A\&A, 397, 899

Sanders, R. H., \& McGaugh, S. S. 2002, ARA\&A, 40, 263

Schlegel, D. J., Finkbeiner, D. P., \& Davis, M. 1998, ApJ, 500, 525

Searle, L., \& Zinn, R. 1978, ApJ, 225, 357

Seigar, M. S., Barth, A. J., \& Bullock, J. S. 2006, ApJ, submitted (astro-ph/ 0612228)

Siegel, M. H., \& Majewski, S. R. 2000, AJ, 120, 284

2006 , in ASP Conf. Ser. 352, New Horizons in Astronomy: Frank N.

Bash Symp. 2005, ed. S. J. Kannappan et al. (San Francisco: ASP), 285

Siegel, M. H., Majewski, S. R., Reid, I. N., \& Thompson, I. B. 2002, ApJ, 578, 151
Stetson, P. B. 1987, PASP, 99, 191 1990, PASP, 102, 932

Stoehr, F., White, S. D. M., Tormen, G., \& Springel, V. 2002, MNRAS, 335, L84

Strigari, L. E., Bullock, J. S., Kaplinghat, M., Kravtsov, A. V., Gnedin, O. Y., Abazajian, K., \& Klypin, A. A. 2006a, ApJ, 652, 306

Strigari, L. E., Koushiappas, S. M., Bullock, J. S., \& Kaplinghat, M. 2006b, Phys. Rev. D, submitted (astro-ph/0611925)

Taylor, J. E., Babul, A., \& Silk, J. 2004a, in ASP Conf. Ser. 327, Satellites and Tidal Streams, ed. F. Prada, D. Martinez Delgado, \& T. J. Mahoney (San Francisco: ASP), 205

Taylor, J. E., Silk, J., \& Babul, A. 2004b, in IAU Symp. 220, Dark Matter in Galaxies, ed. S. D. Ryder et al. (San Francisco: ASP), 91

Tolstoy, E., et al. 2004, ApJ, 617, L119

Tonry, J., \& Davis, M. 1979, AJ, 84, 1511

Valdes, F. 1998, Guide to the NOAO MOSAIC Data Handling Software (Tucson: NOAO)

Walcher, C. J., Fried, J. W., Burkert, A., \& Klessen, R. S. 2003, A\&A, 406, 847

Walker, M. G., Mateo, M., Olszewski, E. W., Pal, J. K., Sen, B., \& Woodroofe, M. 2006, ApJ, 642, L41

Wang, H. Y., Jing, Y. P., Mao, S., \& Kang, X. 2005, MNRAS, 364, 424

Westfall, K. B., Majewski, S. R., Ostheimer, J. C., Frinchaboy, P. M., Kunkel, W. E., Patterson, R. J., \& Link, R. 2006, AJ, 131, 375

White, S. D. M., \& Rees, M. 1978, MNRAS, 183, 341

Wilkinson, M. I., \& Evans, N. W. 1999, MNRAS, 310, 645

Wilkinson, M. I., Kleyna, J., Evans, N. W., \& Gilmore, G. 2002, MNRAS, 330, 778

Wilkinson, M. I., Kleyna, J. T., Evans, N. W., Gilmore, G. F., Irwin, M. J., \& Grebel, E. K. 2004, ApJ, 611, L21

Yanny, B., et al. 2003, ApJ, 588, 824

Zaritsky, D., Olszewski, E. W., Schommer, R., Peterson, R. C., \& Aaronson, M. 1989, ApJ, 345, 759 (Z89)

Zentner, A. R., Kravtsov, A. V., Gnedin, O. Y., \& Klypin, A. A. 2005, ApJ, 629,219 